\title{
A History and Annotated Account of the Benthic Marine Algae of Taiwan
}

\section{JANE E. LEWIS}

and

JAMES N. NORRIS

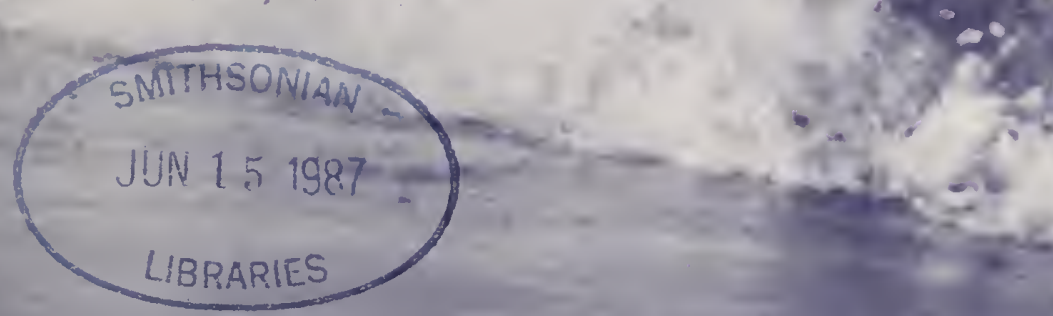

SMITHSONIAN CONTRIBUTIONS TO THE MARINE SCIENCES - NUMBER 29 


\title{
SERIES PUBLICATIONS OF THE SMITHSONIAN INSTITUTION
}

Emphasis upon publication as a means of "diffusing knowledge" was expressed by the first Secretary of the Smithsonian. In his formal plan for the Institution, Joseph Henry outlined a program that included the following statement: "It is proposed to publish a series of reports, giving an account of the new discoveries in science, and of the changes made from year to year in all branches of knowledge." This theme of basic research has been adhered to through the years by thousands of titles issued in series publications under the Smithsonian imprint, commencing with Smithsonian Contributions to Knowledge in 1848 and continuing with the following active series:

\author{
Smithsonian Contributions to Anthropology \\ Smithsonian Contributions to Astrophysics \\ Smithsonian Contributions to Botany \\ Smithsonian Contributions to the Earth Sciences \\ Smithsonian Contributions to the Marine Sciences \\ Smithsonian Contributions to Paleobiology \\ Smithsonian Contributions to Zoology \\ Smithsonian Folklife Studies \\ Smithsonian Studies in Air and Space \\ Smithsonian Studies in History and Technology
}

In these series, the Institution publishes small papers and full-scale monographs that report the research and collections of its various museums and bureaux or of professional colleagues in the world of science and scholarship. The publications are distributed by mailing lists to libraries, universities, and similar institutions throughout the world.

Papers or monographs submitted for series publication are received by the Smithsonian Institution Press, subject to its own review for format and style, only through departments of the various Smithsonian museums or bureaux, where the manuscripts are given substantive review. Press requirements for manuscript and art preparation are outlined on the inside back cover.

Robert McC. Adams

Secretary

Smithsonian Institution 


\title{
A History and Annotated Account of the Benthic Marine Algae of Taiwan
}

\author{
Jane E. Lewis \\ and James N. Norris
}

\section{ISSUED}

JUH $8 \quad 1987$

SMITHSONIAN INSTITUTION

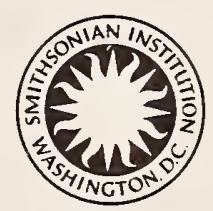

SMITHSONIAN INSTITUTION PRESS

Washington, D.C.

I 987 


\section{A B S T R A C T}

Lewis, Jane E., and James N. Norris. A History and Annotated Account of the Benthic Marine Algae of Taiwan. Smithsonian Contributions to the Marine Sciences, number 29, 38 pages, I figure, 1987. - Records of the benthic marine algae of the Island of Taiwan and neighboring islands have been organized in a floristic listing. All publications with citations of benthic marine green algae (Chlorophyta), brown algae (Phaeophyta), and red algae (Rhodophyta) in Taiwan are systematically arranged under the currently accepted nomenclature for each species. The annotated list includes names of almost 600 taxa, of which 476 are recognized today. In comparing the three major groups, the red algae predominate with $55 \%$ of the reported species, the green algae comprise $24 \%$, and the browns $21 \%$. Laurencia brongniartii J. Agardh is herein reported for Taiwan for the first time.

The history of modern marine phycology in the Taiwan region is reviewed. Three periods of phycological research are recognized: the western (1 866-1905); Japanese (1895-1945); and Chinese (1950-present). Western phycologists have apparently overlooked the large body of Japanese studies, which included references and records of Taiwan algae.

By bringing together in one place all previous records of the Taiwanese marine flora, it is our expectation that this work will serve as a basis for further phycological investigations in the western Pacific region.

OfFICIAL PUBLICATION DATE is handstamped in a limited number of initial copies and is recorded in the Institution's annual report, Smithsonian Year. SERIES COVER DESIGN: Seascape along the Atlantic Coast of eastern North America.

Library of Congress Cataloging in Publication Data

Lewis, Jane $\mathbf{E}$.

A history and annotated account of the benthic marine algae of Taiwan.

(Smithsonian contributions to the marine sciences; no. 29)

Bibliography: p.

Includes index.

Supt. of Docs. no.: SI 1.34: 29

1. Marine algae-Taiwan-Classification-Bibliography. 2. Marine algae-Taiwan-Bibliography. 3.

Marine algae-Research-Taiwan-History. I. Norris, James N. II. Title. III. Series Z5356.A6L48 $1987 \quad 016.5894^{\prime} 0951^{\prime} 249 \quad 86-60025 \mathrm{I}$

[Qk575.T28] 


\section{Contents}

Historical Review .......................... 1

Geographic and Oceanographic Features . . . . . . . . . . . 4

Discussion . . . . . . . . . . . . 6

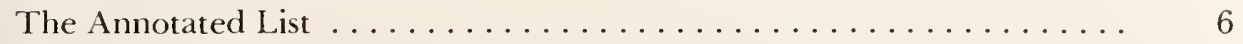

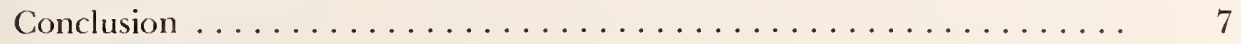

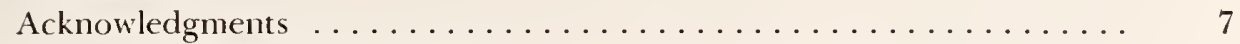

Benthic Marine Algae from Taiwan . . . . . . . . . . . . . . 7

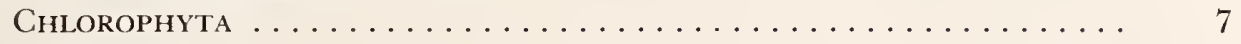

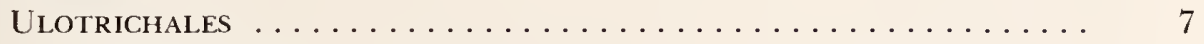

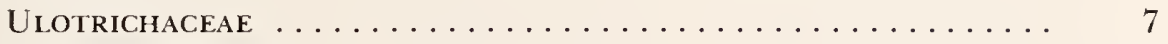

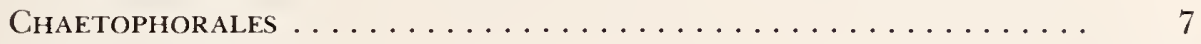

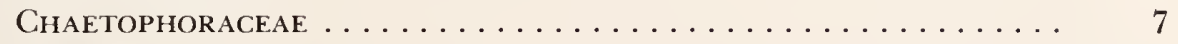

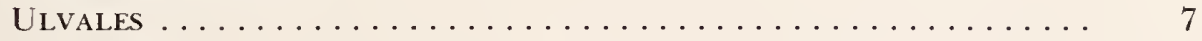

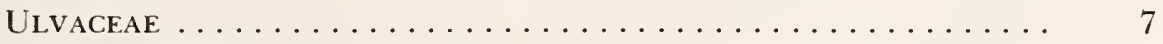

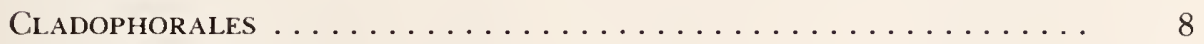

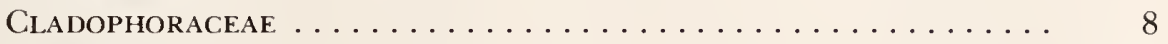

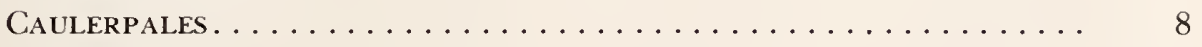

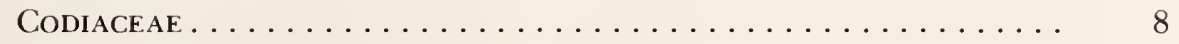

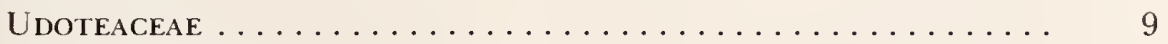

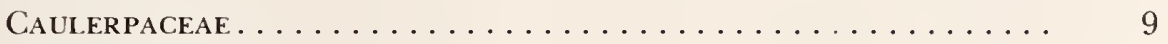

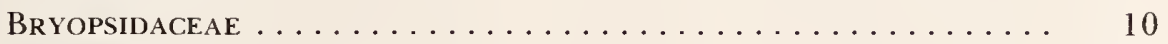

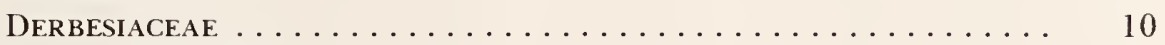

SIPHONOCLADALES ...................... 10

SiPHONOCLADACEAE ................... 10

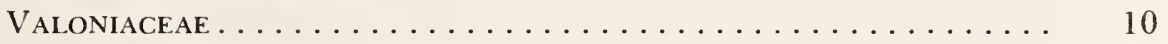

ANADYOMENACEAE ......................... 11

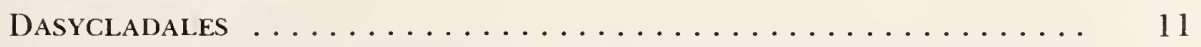

ACETABUlariacEAE ...................... 11

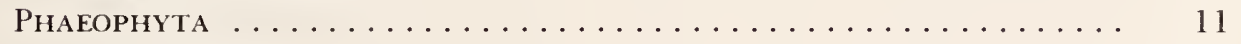

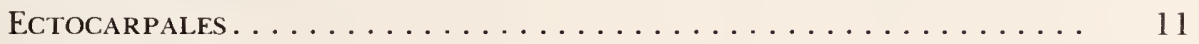

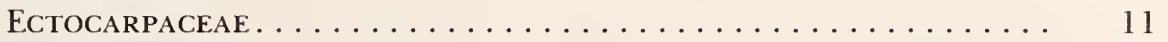

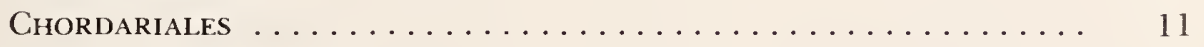

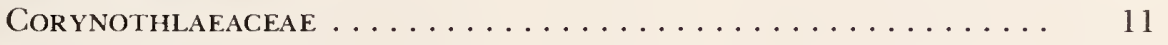

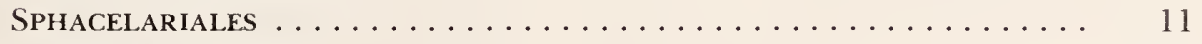

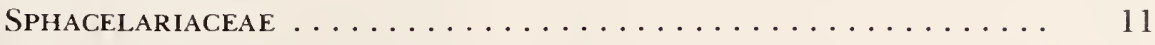

DiCTYOTALES . . . . . . . . . . . . . . . . . . 11

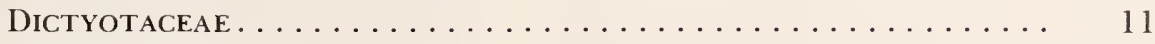

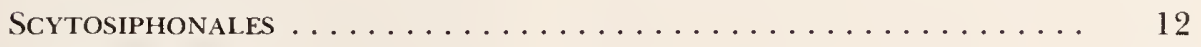

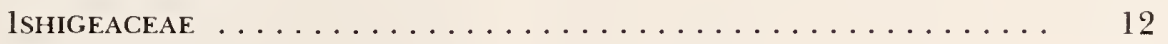

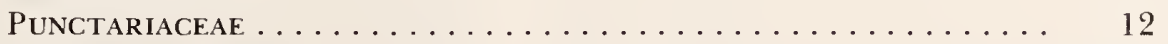

SCYTOSIPHONACEAE .................... 13

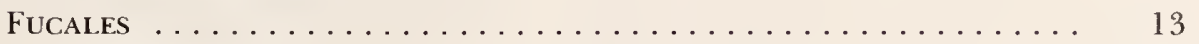

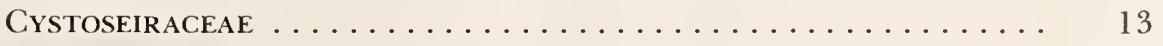

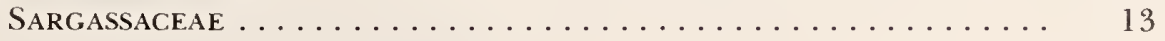


RHODOPHYTA ........................... 14

Goniotrichales ........................ 14

GoniotrichacEAE ....................... 14

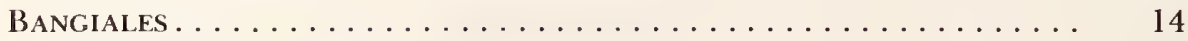

ERYthropeltidACEAE ...................... 14

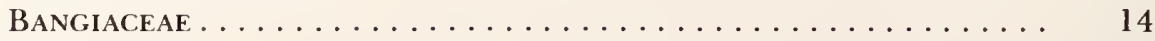

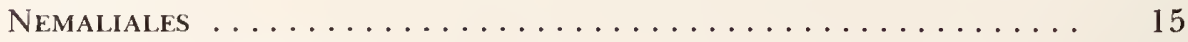

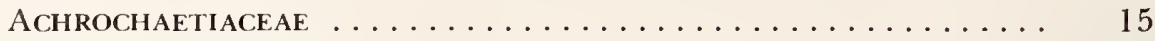

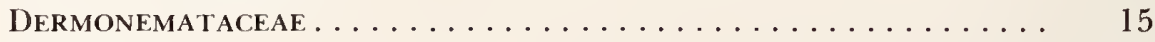

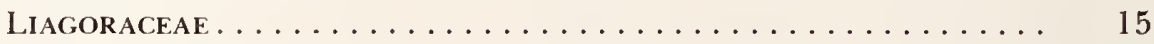

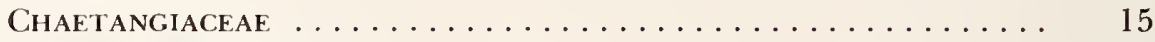

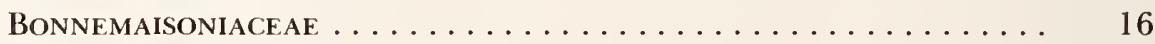

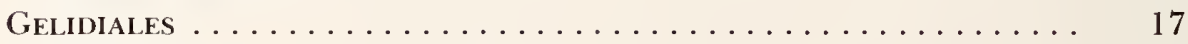

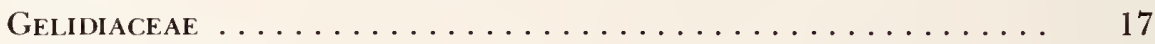

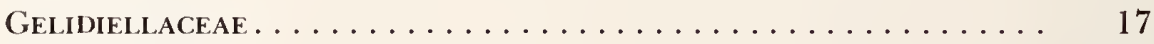

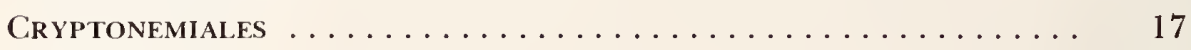

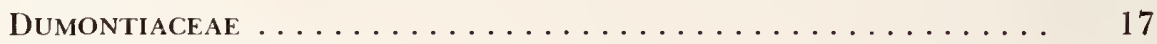

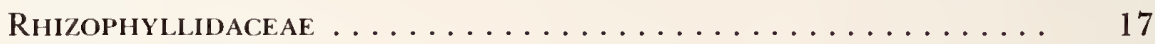

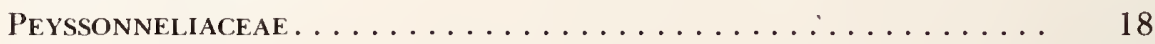

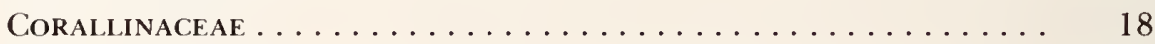

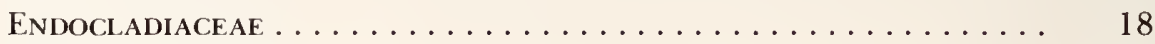

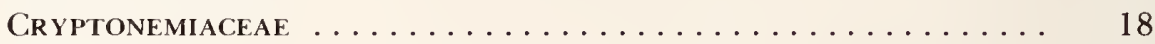

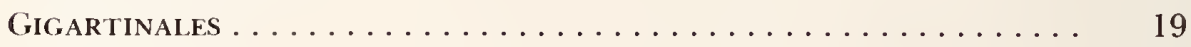

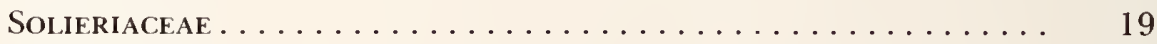

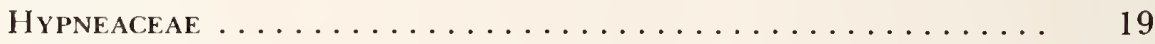

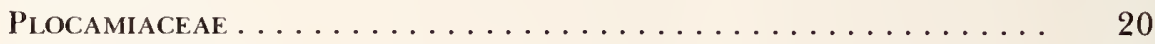

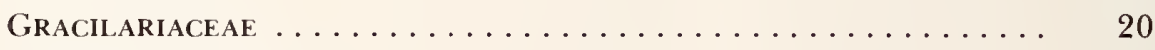

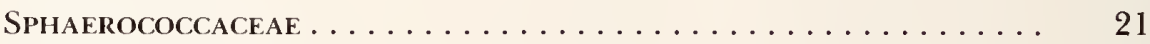

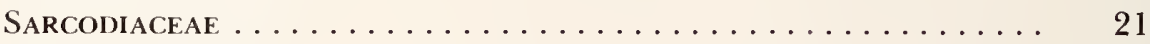

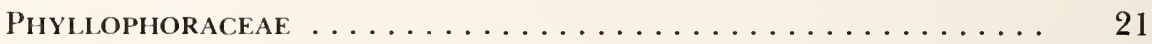

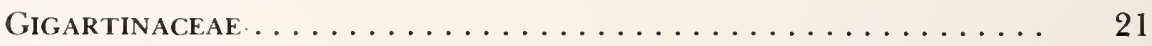

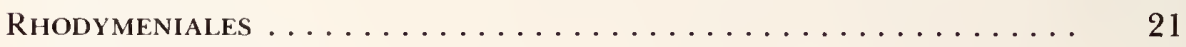

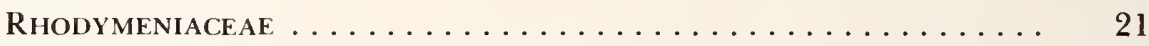

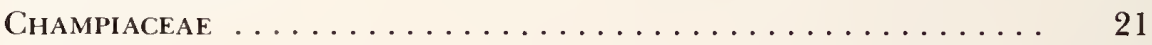

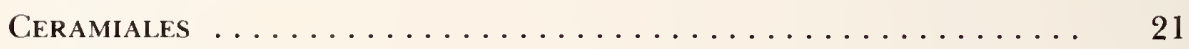

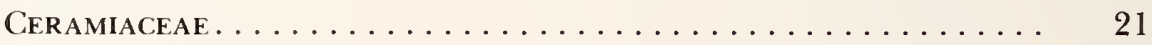

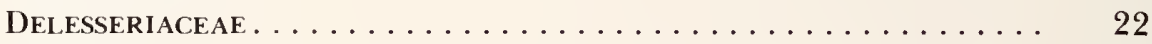

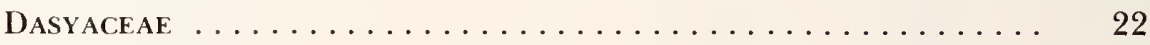

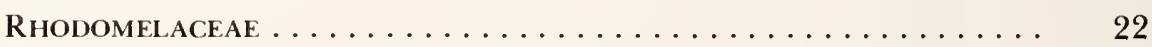

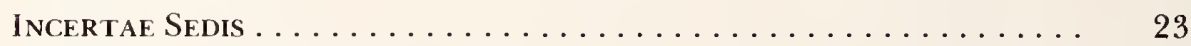

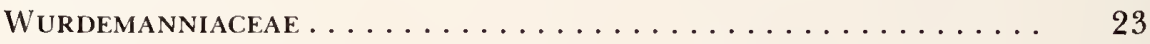

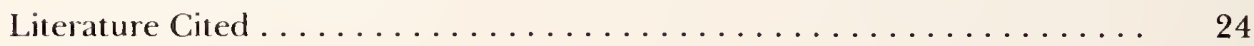

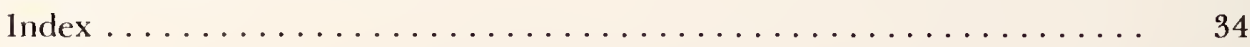




\title{
A History and Annotated Account of the Benthic Marine Algae of Taiwan
}

\author{
Jane E. Lewis and James N. Norris
}

\section{Introduction}

\section{Historical Review}

The history of recognizing, naming, and using marine algae in China extends far back into the early Chinese literature. Although of uncertain antiquity, the specific mention of marine algae goes back at least to the publication of the Er Ya* or Literary Expositor (Tseng and Chang, 1961), a dictionary that dates to the 3rd century B.C. (Needham, 1970). Application of Latin binomials to the Taiwan marine flora, however, began only in the late 19th century (Martens, 1866). After a period of European collections and reports, a period of extensive Japanese studies during the early to mid-20th century followed. The third, or current, period is one in which the activities of Chinese investigators predominate.

The first report of benthic Taiwan algae that followed Linnean taxonomy appeared in Georg von Martens' Die Tange (1866), based on the botanical explorations from the German expedition to East Asia, 1860 to 1862. During this voyage von Martens' son Eduard, chief zoologist for the expedition, collected marine and freshwater algae from Java, the Philippines, Singapore, Taiwan, Hong Kong, and other East Asian areas. In this work, the elder von Martens reported seven marine species from "Tamsui" (Danshui), a northeast Taiwan seaport, including three marine algal species, Ulva lactuca f. lapathifolia, Grateloupia filicina $\mathrm{f}$. filiformis, and Caulacanthus ustulatus var. fastigiatus, not again recorded from Taiwan.

During a second German expedition to East Asia (18861888) Dr. Warburg made phycological collections that were later published by Heydrich (1894) and De Toni (1895,

Jane E. Lewis, Department of Botany, University of Hawaii, and East-West Center, Honolulu, Hawaii 96822. James N. Norris, Department of Botany, National Museum of Natural History, Smithsonian Institution, Washington, D.C. 20560.
1905). This expedition covered the East Indian Ocean, the Mollucca Islands, China, Java, the southern Japanese islands, and a brief stop at Taiwan. Taiwan collection locations were noted as Jilong (a northeast seaport), south Taiwan, the east coast and "Long-kiau" on the south coast.

Among Warburg's Taiwanese algal collection, Heydrich identified 43 taxa, consisting of 24 red (Rhodophyta), 12 brown (Phaeophyta), and 7 green (Chlorophyta) species. In continued studies of the algae from this expedition, De Toni (1895) reported 36 taxa from Taiwan and in 1905 another two species. As a result of these early European investigators, over 60 marine benthic algae were reported for the Taiwan region, including many new to science.

The history of the exploration of Taiwan marine algae, as with other sciences and indeed all aspects of life, was greatly shaped during the 50 -year period, beginning in 1895, of Japanese political dominance in Taiwan. Voluminous studies on the Japanese flora and fauna, then including Taiwan, were undertaken. It was during this time that exploration of the Taiwan marine flora was most intensive, dominated by the work of K. Okamura (e.g., 1900-1902, 1907-1942) and his student, Y. Yamada (1930-1944).

The first publication of this period, "New or Little Known Algae From Japan" (Okamura, 1895), included two new. records of species from Taiwan. Many publications specifically addressed the Taiwan flora (Okamura, 1915b, 1931, 1935b; Oshima, 1915; Ariga, 1919, 1920, 1921; Horikawa, 1919; Yamada 1925a, 1925b, 1936a; Yamada and Tanaka, 1934; Tokida, 1941), but most references to the Taiwan flora were included as distributional notes or collection sites within general Japanese floristic works (Okamura, 1930, 1936; Yamada, 1928, 1934). Three important series con-

* Throughout this text, Chinese place names and words not enclosed in quotation marks are spelled according to the Pin Yin romanization system. 
taining numerous, but scattered, references to Taiwan algae are Illustrations of the Marine Algae of Japan (Okamura, 1900-1902) and Icones of Japanese Algae (Okamura, 1907, 1909, 1913, 1915a, 1921, 1923, 1932, 1934b, 1935a, 1937, 1942); Yamada's "Notes on Some Japanese Algae" (1930, 1931a, 1932b, 1933, 1935, 1936b, 1941, 1944b); and Yendo's “Notes on Algae New to Japan” (1909, 1914, $1915,1916 \mathrm{a}, 1916 \mathrm{~b}, 1918)$.

From an early time the Japanese were interested in floristic comparisons, sometimes speculating on the reasons for disjunct species distributions or factors that might cause adjacent areas to have divergent floras (Ariga, 1920, 1921; Okamura, 1915b, 1919). One of the first comparative phytogeographic studies of the western Pacific region (Yamada, 1926) included Taiwan as one of its eight floristic areas. Of the 42 taxa compared in the region, 16 were present in Taiwan.

Japanese monographs included new or additional records of taxa for Taiwan. Among these were studies of the Corallinaceae (Segawa, 1941; Yendo, 1902) and the Florideophyceae (Tanaka, 1944), and the genera Gelidium and Pterocladia (Okamura, 1934a), Galaxaura (Tanaka, 1935, 1936), Hypnea (Tanaka, 1941), Laurencia (Yamada, 1931b, 1936c), Liagora (Yamada, 1938a), Porphyra (Ueda, 1932), and Sargassum (Yamada, 1942).

Many of the papers written specifically on Taiwan's marine flora are in Japanese. Ariga (1919) describes a 14-day collecting trip and lists 43 taxa collected. Horikawa's (1919) "Marine Algae of Taiwan" reports $20 \mathrm{red}, 10 \mathrm{brown}$, and 15 green species, based on determinations by Okamura of specimens that had been collected by Horikawa, Nagasawa, Maki, and their students at locales around Taiwan, including Danshui and Jilong in the north, Elanbi (Olanpi) in the south, Xiao Liuqiu (Shao Liou Chou) and Lan Yu, and the Penghu (= Pescadores) 1slands. In another study a year later, Ariga (1920) reported 69 taxa for the Penghu Islands and compared them with his records from "Amoy," a small island off Fujian (= Fukien) Province, southeast China coast. Although only about $135 \mathrm{~km}$ of the shallow Taiwan Strait separate the two sites, the floras were found to be drastically different in composition. Even when the same species occurred in both places they often exhibited marked morphological or seasonal differences. 1t was speculated that tidal fluctuation or substrate type may have effected these differences. In determining species names, Ariga (1919, 1920) relied heavily on the papers of Yendo and Okamura.

The papers of Ariga (1919, 1920) and Horikawa (1919) were subsequently overlooked by later authors, perhaps due in part to having been written in Japanese. Both 1919 papers use the Latin scientific names and authors of the species followed by Japanese names. Ariga, in his subsequent paper (1920), uses these Japanese names almost exclusively. Few cases are known in other languages where common names apply solely to a single species. In the course of our research, however, these Japanese names were found to be a special case because they are not common names but a Japanese version of the scientific name (Lai Chuen-fu, personal communication). When Latin binomials were introduced to Japan, the Japanese erected equivalent Japanese scientific names, one for each taxon, which were different from the Japanese common names. Because the Japanese names are listed with their Latin binomials in Okamura's flora (1936), it has been possible to translate Ariga's names into the binomial form for inclusion in the current listing.

Yamada's studies on the Chlorophyceae (1925a) and the Phaeophyceae (1925b) of Taiwan, representing his undergraduate thesis at Tokyo University under K. Okamura, are frequently cited in later publications. Most of the specimens of these papers were collected by Yamada during a short spring collecting trip to the north and south coasts of Taiwan and to the Penghu 1slands, with a few additional specimens provided by T. Aoki, then a government official in Taiwan.

Economic considerations have also contributed to identification of Taiwan's marine flora. Some papers were devoted to the agarophytes in Taiwan at a time when the Japanese seaweed industry was flourishing (Okamura, 1915b, 1935b; Oshima, 1915). Taiwan's east coast was thought to possibly have a richer agarophyte flora than north Taiwan or any other coast in Japan. However, due to the presence of unfriendly aborigines (Okamura, 1915b), this hypothesis was not investigated until much later, and was found to be incorrect (Fan, 1951).

Several papers discuss Taiwan algae used as foods. The island of Lan Yu (also called Kôtosho, Botel Tobago, or Orchid Island) has attracted much attention because of its aboriginal population, unique biology, and its close proximity $(61 \mathrm{~km})$ to the island of Taiwan. An issue of the Bulletin of the Biogeographical Society of Japan (1931, vol. 2, no. 2) was devoted to the anthropology and biology of Lan Yu, with Okamura (1931:95-122) reporting 92 taxa of benthic algae from collections made by S. Segawa during one summer month in 1930 . Only about one-third of these algae (Okamura, 1931) were also known to be on both Taiwan and Lan Yu. The Lan Yu flora was considered to be Indo-Pacific in nature, whereas no specific comments were made on the affinities of the Taiwan 1sland marine flora. Of the many algae presumably eaten by the "Yami" tribe on Lan Yu, Okamura listed 12 (Carpopeltis formosana, Laurencia sp., Acanthophora orientalis, Halymenia durvillaei var. formosa, Hypnea seticulosa?, Nemalion pulvinatum, Chondria armata, and Dermonema dichotoma), also giving a Japanese transliteration of their traditional Yami names. Subsequently, Tokida (1939) published an almost identical list of algae eaten by the "Ami" people on Taiwan with the same common names.

An identification book with photographs of Japanese seaweeds published during this period (Higashi, 1934) in- 
cluded 12 records of Taiwan taxa. With the high scholarly level characteristic of the Japanese picturebook genre, specific names are generally considered accurate. Because these books contain records of Taiwan algae often not previously published, it is believed that distributional citations were based on Japanese herbarium material.

The Japanese flora Nippon Kaiso-shi (Okamura, 1936) contains numerous reports of algal species in Taiwan. This is an invaluable reference for the identification of benthic marine algae throughout the northwestern Pacific region.

In addition to the extensive Japanese publications of the period 1895-1945, some Chinese and western papers also included accounts of the Taiwan marine flora. A.D. Cotton (1915) reported nine taxa from Taiwan. Specimens of Sargassum were verified by K. Yendo, who visited the Royal Botanic Garden, Kew, while the paper was in preparation.

The lack of scientific communication between the East and West is perhaps characterized by J. Tilden's (1929) comments in "The Marine and Freshwater Algae of China." Although Professor Tilden claims to record "all species previously noted by investigators" for the algae of China, her account of Chinese phycological history only cites one non-western reference, a short paper by Okamura (1913) on "Chinese Edible Nostoc." She clearly agrees with Cotton (1915) that "Formosa [now known as Taiwan] ... though belonging to Japan, must geographically be included with China." Though aware of Martens' (1866) account of algae, Tilden considered his determinations to be unreliable, and her account of the Taiwan marine flora was limited to only the nine taxa Cotton (1915) had reported.

Apparently unaware of the Japanese literature of 18951928, Tilden listed only 92 species of marine algae from the entire Chinese region, as "all that has been done in the study of Chinese algae." If the Japanese literature (e.g., Okamura, 1909-1926) and other accounts of European expeditions had been included, she would have found over 350 published records of more than 200 species for Taiwan alone by 1928. 1t is easy today to see the inaccuracy of Tilden's assessment; however, her conclusions were understandable considering the barriers of language, culture, and communication at that time.

Professor C.K. Tseng, as a young botany instructor from Fujian Province, made extensive collections in the 1930's throughout China. In studies of marine Chlorophyceae from Hainan lsland (1936), Chinese Chaetangiaceae (1941b), Hong Kong Polysiphonia (1944), and "New and Unrecorded Marine Algae of Hong Kong" (1945), Tseng included several Taiwan collections, many of them new records.

The first publication by Chinese marine botanists on Taiwan after the Japanese occupation came from the Taiwan Fisheries Research Institute. Y.F. Shen and K.C. Fan (1950) compiled much of the earlier literature and studied their own collections as well as specimens from the herbar- ium at the National Taiwan University made from Kôtôsho by Y. Yamamoto, which resulted in a list of 62 green, 50 brown, and 142 red taxa. Locations were given as "Taiwan," "Kôtôsho" (Lan Yu), "Kasyoto" (Lu Dao) and the "Pescadores" (Penghu 1slands).

Fan (1951) described eight species and two forms of the economic genera Gelidium and Pterocladia from Taiwan, with English translations of Dr. Okamura's (1935) four new taxa. Gelidium and Pterocladia were found to be restricted to the north and northeast part of the island. The distributions of the various species were compared and found to belong to two distributional groups (a disjunct north-south distribution), but Fan concluded that "[the] chief factors that delimit the mutual exclusion of these species are at present uncertain."

A list of edible marine algae (Fan, 1952) from Taiwan included 30 taxa used by Taiwan aborigines as well as those with a long Chinese tradition, with brief descriptions and localities of the algae, preparation methods, and a table of their Chinese and aboriginal names. Many species were noted to be commonly occuring only in the spring, though no reason was suggested.

Since 1960, Y.M. Chiang (now a professor at Taiwan National University), has been a major investigator of the Taiwan marine algae. Studies on floristics (Chiang, 1960, 1962a,b, 1973a,b), taxonomy (Chiang, 1981; Chiang and Chen, 1982; Yang and Chiang, 1982), reproduction (Chen and Chiang, 1982; Chiang, 1969, 1970, 1971, 1972; Chiang and Chen, 1982), and aquaculture (Chiang, 1981, 1982; Chiang and Chou, 1980; Liaw and Chiang, 1979; Nelson et al., 1983) have been the focus of his phycological contributions.

Professor Chiang began phycological publications (as a student of Y.F. Shen, Botany Department, National Taiwan University) with his two-part "Marine Algae of Northern Taiwan," the blue-green, brown, and green algae (1960), and the red algae (1962a). Included in these works were information on collection locations, habitat notes and seasonality of the major algae, identification keys, and taxonomic summaries of each of the 96 species.

After a field trip of a few days to Penghu, Chiang (1962b) recorded 26 species and noted the fragmentary nature of earlier Japanese records in the Penghu lslands. Dr. Chiang began working on Taiwan's phytogeographic affinities with "Notes on Marine Algae of Taiwan" (1973a), which included north-south distributional observations and new records of six green, six brown, and nine red algae for Taiwan and its offshore islands. "Studies on the Marine Flora of Southern Taiwan" (1973b) reported on the algae at four southern localities and compared them with the north, finding that "some northern species do not occur in the southern waters. On the contrary, there are quite many species which occur in southern Taiwan but not in the northern regions." The southern flora was also considered 
to be more depauperate than the northern and unique within Taiwan in having typically tropical elements, such as Bornetella and Neomeris. The water temperatures, warmer and less variable throughout the year in the south, were suggested to be partially responsible for this difference. Because Okinawa, located slightly to the north and east of Taiwan, shares tropical genera with southern Taiwan, Chiang also suggested that the Kuroshio (or Japanese) Current is likely to be a major factor in distribution.

A later series of papers focus on aspects of morphology and reproduction (Chiang and Chou, 1980; Chen, Chiang, and Chiang, 1981; Chen and Chiang, 1982; Chiang, 1982; Chiang and Chen, 1983) and vegetative reproduction in a brown alga (Chiang and Chou, 1980). These papers provide taxonomic information, some of which is new for the Taiwan flora, and others that substantiate previous records. Taxonomic studies on Sargassum (Chou and Chiang, 1981), Liagora (Chiang and Chen, 1982), and Gracilaria (Yang and Chiang, 1982) provide additional listings with distribution, morphology and seasonality information.

Aquaculture in Taiwan is a well-developed business principally of shellfish and fin fish, and in the last 10 years the culture of marine algae (Chueh and Chen, 1982), especially Gracilaria (Chen, 1976; Shang, 1976; Michanek, 1978; Ryther, 1979; Tseng, 1981a,b; Chiang, 1981; Doty, 1983), and Porphyra (Chiang, 1982), has received more attention from both growers and taxonomists. Of the numerous publications on seaweed culture, those providing records of native species in Taiwan were most appropriate for inclusion in this listing.

In a review of Gracilaria culture in Taiwan, Chiang (1981) and Hansen et al. (1981) provided taxonomic information on the three native species cultured (G. edulis, $G$. gigas, and $G$. verrucosa) and the techniques employed. The seaweed aquaculture program, centered in the southwest of Taiwan, began in 1961, and by 1979 about 12,000 tons of the dry seaweed were being harvested, predominately for the domestic agar industry, and about 120 tons of fresh seaweed went as feed to abalone farms.

Gracilaria is the major source of agar-agar, along with some Gelidium. Reports on seasonal variation of agar quality and quantity produced in Taiwan (Yang, 1982; Yang et al., $1981)$ provide records on the native agarophyte flora, and a comparison of agar from species in Taiwan and Micronesia (Nelson et al., 1983) provides information on Taiwan sites of natural populations.

In contrast to Gracilaria, Porphyra culture has been smallscale and irregular. Cultivation of local species in the Penghu lslands began in 1968, but studies on the life history of the local species were not initiated until 1975 (Chiang, 1982), when the "Conchocelis-stage" of Porphyra and monospore formation were investigated. This study and two on Conchocelis culture (Chiang and Chou, 1980; Liaw and Chiang, 1979) also provide taxonomic information on native Taiwan taxa.
Alginates, a family of chemicals used in food preparation and with a wide variety of industrial uses, which conventionally are extracted from cold-water kelps, may become an industry in Taiwan, using a tropical brown alga. Research has been conducted (Liu, 1982) on improving the quality of alginates from Sargassum duplicatum, a species growing naturally along the shoreline of the southern tip of Taiwan.

Green algae are also a part of Taiwan's aquaculture industry. Liu (1982) mentions use of Chlorella. Included in a report on chemical analysis and utilization of Monostroma $(\mathrm{Wu}, 1982)$ is information on the two native species and their distribution.

The study of biological activity and natural-product chemistry (e.g., Norris and Fenical, 1985) is a recent research interest in Taiwan. For example, in a recent paper, "Pharmacological Properties of Some Taiwan Seaweeds" (Su et al., 1982), 30 species from Taiwan were screened for antimicrobial activity.

\section{Geographic and Oceanographic Features}

The area encompassed in this study includes the main island of Taiwan and a few of its 14 associated islands as well as the 64 islands of the Pescadores Archipelago (Figure $1)$. The islands most commonly referred to in the phycological literature are those of Taiwan (= Formosa) $\left(21^{\circ} 53^{\prime}-\right.$ $25^{\circ} 18^{\prime} \mathrm{N}, 120^{\circ} 1^{\prime}-122^{\circ} 0^{\prime} \mathrm{E}$ ), Orchid lsland (= Kôtôsho or Lan $\mathrm{Yu})\left(22^{\circ} 0^{\prime}-22^{\circ} 5^{\prime} \mathrm{N}, 121^{\circ} 36^{\prime}-121^{\circ} 30^{\prime} \mathrm{E}\right)$, Green Island (= Kasyoto or Lu Dao) $\left(22^{\circ} 38^{\prime}-22^{\circ} 41^{\prime} \mathrm{N}, 121^{\circ} 28^{\prime}-\right.$ $\left.121^{\circ} 30^{\prime} \mathrm{E}\right)$ and the Pescadores (= Penghu Islands) $\left(23^{\circ} 11^{\prime}-\right.$ $\left.23^{\circ} 46^{\prime} \mathrm{N}, 119^{\circ} 18^{\prime}-119^{\circ} 42^{\prime} \mathrm{E}\right)$. The most frequented collecting sites on the Island of Taiwan have been Olanpi at the southern tip, "Tai Dung" on the east coast, and the general area of the northern tip. Bisected by the Tropic of Cancer, the Island of Taiwan is considered both tropical and subtropical. Geologically it is a continental island with a mostly sedimentary origin, and it has a coastline of roughly $1600 \mathrm{~km}$.

Oceanographic conditions around the island vary in topography, temperature, and currents. The Taiwan Strait is shallow (60 $\mathrm{m}$ average depth) and turbid, extending westward from Taiwan some 140 to 200 nautical miles to the southeast coast of mainland China. Within the Strait, the Penghu lslands are some $40 \mathrm{~km}$ from Taiwan. Tidal currents around these islands reach almost 6 knots in places (USDD Nautical Map \#94060). In the East China Sea northeast of Taiwan is the Ryukyu Island chain, beyond which are the main islands of Japan. To the south the Bashi Channel separates Taiwan and the Philippines. The east coast, often precipitous, marks the eastern edge of the continental shelf. The sharp drop-off continues some 4000 $\mathrm{m}$ below sea level, reaching the floor of the Philippine Basin. Along the southeast coast are areas of upwelling, with colder, nutrient-rich waters.

Many benthic marine algae are found growing in the 


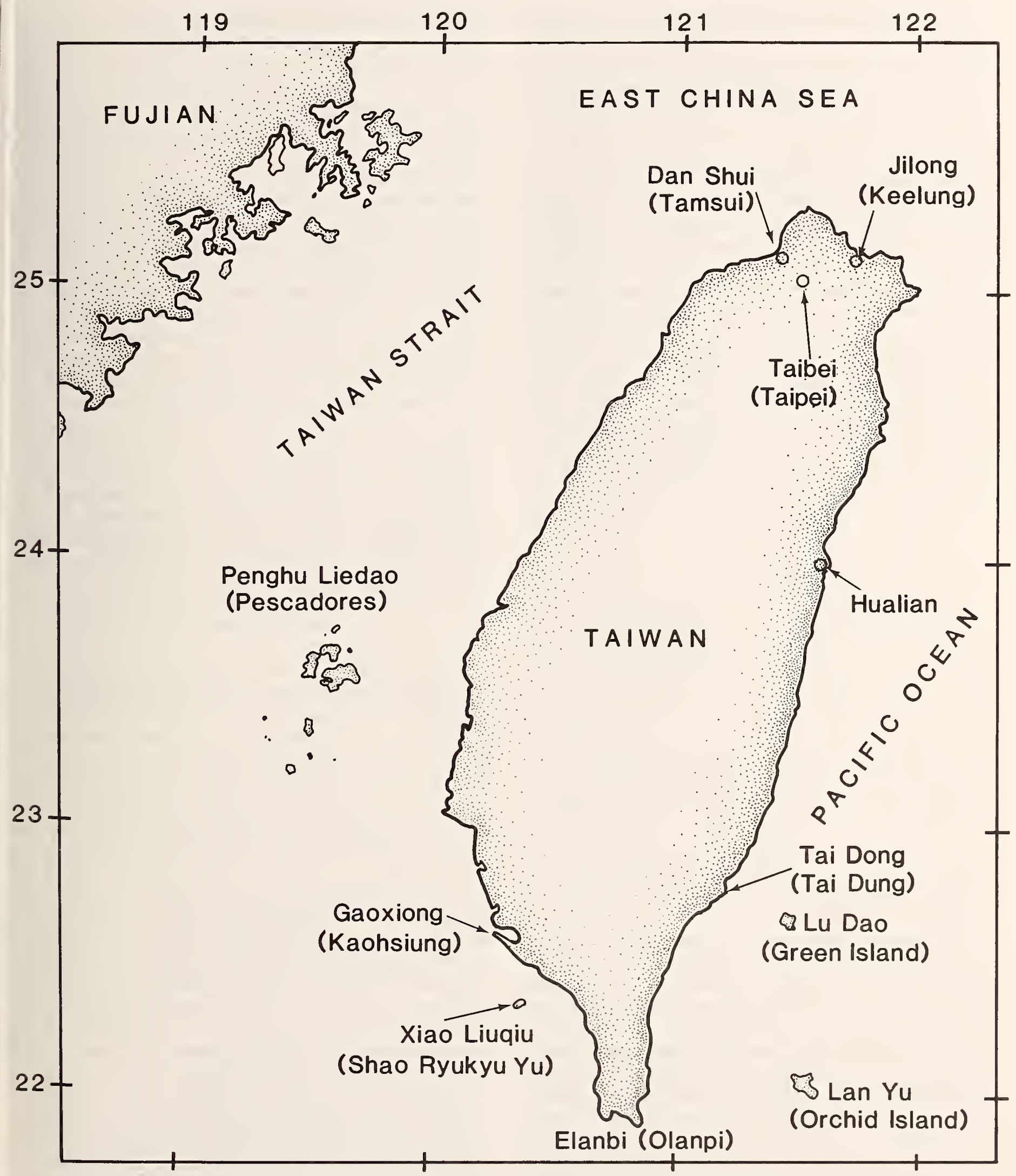

FigURE 1.- Map of Taiwan, showing islands and locales most commonly referred to in the phycological literature. 
nearshore coastal waters. Shoreline conditions in Taiwan vary from sandy to rocky shores with murky water in the north, to coral reefs with clear swift water in the south. To the east are precipitous cliffs and rough waters with deepocean upwellings along the coast and, in contrast, level, calm expanses of intertidal mud and sand flats along the western shoreline.

The two main currents affecting Taiwan are the Kuroshio (or Japanese) Current and the Taiwan coastal current. The Kuroshio Current brings water of high temperature and salinity up from the Philippines and the equatorial region toward southern Taiwan where it branches. The stable, main branch runs past the east coast of Taiwan at an average velocity of 30 to 40 nautical miles per day toward Japan (USDD Nautical Map \#94010). A smaller branch, subject to seasonal variability, veers west through the Taiwan Strait.

Flowing south along the China mainland coastal region, the Taiwan coastal current carries colder water from north to south, and is strong in summer and weak in winter. Two smaller seasonal currents, the northeast monsoon current and the southwest seasonal current, result from an interaction between the two major currents and the prevailing winds.

\section{Discussion}

While compiling the records of Taiwan algae from the numerous publications, efforts were made to find the correct name as well as the valid date and place of publication for each of the taxa in the annotated list. Unfortunately, phycologists do not have a modern source for specific names such as Index Kewensis (Jackson et al., 1893 to date; see Stafleu and Cowan, 1979:397-398, for complete listing) for phanerogams, or Index Filicum (Christensen, 1905, 1906; see Stafleu and Cowan, 1976:501-501, for complete listing) for ferns. Our effort has shown how great the need is for such an index of algal species names, such as has recently been done for the generic names of fossil and living plants (Farr et al., 1979). In the absence of such a reference, we found the earlier works of De Toni (1895-1924) and Dawson (1962) helpful.

The Taiwan algae project was undertaken recognizing that a single bibliographic source on the Taiwan marine flora would facilitate identification of the algae and their distribution and would encourage further research within the region. It was evident that the existing literature was widely dispersed and not adequately referenced in most phycological bibliographies. It became necessary to carefully research the east Asiatic algal literature, particularly from Japan and China, and the whole Pacific region, in order to locate information on Taiwan algae. Work on this compilation commenced while the first author was employed at the Fisheries Biology Laboratory, Institute of Zoology, Academia Sinica, Taiwan. Additional extensive searches were necessary to locate original publications to establish proper citation of the binomial and to subsequently determine the current taxonomic and systematic status of each taxon. For this work, the E. Yale Dawson Phycological Library and Department of Botany libraries of the National Museum of Natural History, Smithsonian 1nstitution, were invaluable.

The annotated list stands as a compilation of reports of attached, benthic marine taxa occurring naturally in the region of Taiwan. Efforts were taken to locate all relevant literature, and although it is possible that additional reports can be found, the present work represents the most comprehensive compilation available for the region. It must be emphasized that records are taken from published papers and that the accuracy of these identifications has not been confirmed by herbarium investigations; reported names of taxa are simply noted and arranged systematically following current convention. Verification of the determinations awaits future investigation. Some indication of accuracy may, however, be gained from the frequency and dates of the reports for any given taxon. For example, the three taxa reported by Martens (1866) that have not again been recorded may be considered in need of verification. Nevertheless, it is felt that the present list reasonably reflects the general composition of the Taiwan marine flora.

\section{The Annotated List}

There are 476 taxonomic entries in this list, systematically arranged following Abbott and Hollenberg (1976) for the red algae, and Lobban and Wynne (1981) for the brown and green algae. Below family level, genera and species within each genus are listed alphabetically. Each entry consists of (1) the currently accepted taxon name, with its author(s), and date and page of valid publication, and (2) an alplabetical list of the investigators who have reported the taxon present in the Taiwan region.

Orthographic errors in publication of taxa were found to have occurred nine times in this list (M.J. Lai and D.H. Nicolson, personal communication). These were simply corrected, with the original spelling in single quotations following "Recommendation 50F.1" of the International Code of Botanical Nomenclature (Voss et al., 1983).

In some cases there are additional names within the entry. In reporting a taxon from Taiwan, authors sometimes used names that have since been considered taxonomic or nomenclatural synonyms, or have been re-determined by later investigators. In all cases, the actual names used by the authors for their algal records from Taiwan are retained in this list. However, when those names differ from the currently accepted ones, they are included in the alphabetical list of references to the taxon. 1t is hoped that by preserving the original names, the concepts held by the authors will be 
indicated and later taxonomic and nomenclatural changes will be more easily integrated into this list.

\section{Conclusion}

The marine flora of this region appears to have tropical, subtropical, and temperate elements. Many authors have commented on the diversity of floral elements within this region (e.g., Ariga, Chiang, Horikawa, Okamura) and have speculated on the possible causes. As noted earlier, water quality and substrate vary greatly in the region, no doubt affecting floral elements. Southern Taiwan and especially the two southeast islands Lan Yu and Lu Dao are referred to as having a tropical/subtropical flora and the north and west as having a temperate flora, judged by the abundance of brown algae and rarity of red algal elements. These correlations are deserving of further study. Indications are that a number of physical and biotic factors interact to create this diversity of floristic types exhibited in the Taiwan region.

Based on written reports from the literature, this study makes available much previously inaccessible information basic to all phycological studies of the East Asiatic region. This comprehensive list of marine algae, treated in both historical and modern systematic contexts, constitutes a basis for further systematic and ecological investigations and biogeographical comparisons among the East Asian and
Pacific areas, and serves as a possible framework for a marine flora of Taiwan.

\section{Acknowledgments}

The first author is very grateful to Jang Kun-Hsiung, Director of the Academia Sinica Zoology Institute and its Fisheries Biology Laboratory, Taipei, Taiwan, for the time and support to initiate this project, Chiang Young-Meng, Oceanography Department, National Taiwan University, Taipei, for references and encouragement throughout the project, and the Department of Botany and the Office of Fellowships and Grants, Smithsonian Institution, for support and use of the libraries, laboratory, and computers. A special appreciation is due to Isabella A. Abbott for her time, guidance, and patience and for the use of her personal library. Japanese translations were kindly provided by Lai Chuen-fu, Rika Taga, and Mason E. Hale. We thank Lai Ming-Jou and Dan H. Nicholson for discussions on nomenclature, and for checking the unpublished algal-name file at the University of California, Berkeley, J. Olsen-Stojkovich and P.C. Silva. For comments on the text, thanks go to J. Berdach, M.I. Cannon, A. Medeiros, and K.E. Bucher. Our appreciation to 1.A. Abbott, K.E. Bucher, Y.M. Chiang, M.S. Doty, M.M. Littler, H.E. Robinson, and C.K. Tseng for reviewing the paper.

\section{Benthic Marine Algae from Taiwan CHLOROPHYTA}

\section{ULOTRICHALES}

\section{ULOTRICHACEAE}

Ulothrix flaccida (Dillwyn) Thuret in Le Jolis, 1863:56. Taniguti, 1976.

\section{CHAETOPHORALES}

\section{Chaetophoraceae}

Endophyton ramosum Gardner, 1909:372. Chiang, 1973a.

\section{ULVALES}

\section{ULVACEAE}

Enteromorpha clathrata (Roth) Greville, 1830:181. Chiang, 1960; Fan, 1953a; Okamura, 1931, 1936; Shen and Fan, 1950; Tokida, 1939.

Enteromorpha clathrata var. crinita (Roth) Hauck, 1884:429.
As "E. crinita": Ariga, 1920; Chiang, 1960; Chihara, 1970; De Toni, 1895; Fan, 1953a; Heydrich, 1894; Okamura, 1936; Segawa, 1974; Shen and Fan, 1950; Tokida, 1939.

Enteromorpha compressa (Linnaeus) Greville, 1830:180. Chiang, 1960; Fan, 1953a; Okamura, 1931, 1936; Shen and Fan, 1950; Taniguti, 1976; Tokida, 1939; Yamada, 1925a, 1926, 1950; Yoshikawa and Yoshikawa, 1977.

Enteromorpha intestinalis (Linnaeus) Link ex Nees, 1820:5. Ariga, 1919; Chiang, 1960, 1973b; Fan, 1953a; Horikawa, 1919; Shen and Fan, 1950.

Enteromorpha linza (Linnaeus) J. Agardh, 1883:134. Chiang, 1960, 1973b; Chiang et al., 1974. As "Phycoseris lanceolata var. angusta": Martens, 1866.

Enteromorpha prolifera (O.F. Müller) J. Agardh, 1883:129. Chiang, 1960; Fan, 1953a; Shen and Fan, 1950; Taniguti, 1976.

Enteromorpha sp.

Chiang, 1973b.

Monostroma latissimum (Kützing) Wittroch, 1866:33.

Chiang, 1973a, 1973b; Okamura, 1935b; Rho, 1958. 
Monostroma nitidum Wittrock, 1866:41.

Chiang, 1960; Fan, 1953a; Okamura, 1936; Shen and Fan, 1950; Taniguti, 1976; Yamada, 1925a, 1925b, 1934, 1950.

Ulva angusta Setchell et Gardner, 1920:283.

Chiang, 1973a; Okamura, 1935b.

Ulva conglobata Kjellman, $1897 \mathrm{~b}: 10$.

Ariga, 1920, 1921 ; Chiang, 1960, 1962a; Shen and Fan, 1950; Taniguti, 1976; Tokida, 1939; Yamada, 1925a; Yoshikawa and Yoshikawa, 1977.

Ulva fasciata Delile, 1813:155.

Chiang, 1960, 1973b; Fan, 1953a; Shen and Fan, 1950;

Tokida, 1939; Tseng, 1983; Yamada, 1935; Yoshikawa and Yoshikawa, 1977.

Ulva japonica (Holmes) Papenfuss, 1960:309.

As "Letterstedtia japonica": Chiang, 1973a; Okamura, 1935b; Taniguti, 1976.

Ulva lactuca Linnaeus, 1753:1 163.

Chiang, 1960, 1973b; Fan, 1953a; Heydrich, 1894; Okamura, 1930, 1931; Shen and Fan, 1950; Su et al., 1982; Yamada, 1950; Yoshikawa and Yoshikawa, 1977.

Ulva lactuca f. lapathifolia (Areschoug) Hauck, 1884:437. As "Phycoseris lapathifolia": Martens, 1866.

Ulva pertusa Kjellman, $1897 \mathrm{~b}: 4$.

Ariga, 1919, 1920; Chiang, 1960, 1973b; Fan, 1953a; Horikawa, 1919; Okamura, 1921; Rho, 1958; Shen and Fan, 1950; Taniguti, 1976; Tokida, 1939, 1954; Yamada, 1925a; Yoshikawa and Yoshikawa, 1977.

Ulva reticulata Forsskål, $1775: 187$.

Arasaki, 1964; Ariga, 1920; Chiang, 1960, 1973b; Fan, 1953a; Okamura, 1936; Rho, 1958; Segawa, 1974; Shen and Fan, 1950; Tokida, 1939; Tseng, 1936; Yamada, $1925 a$.

Ulva rigida C. Agardh, 1823:410.

Ariga, 1920; Yendo, $1916 \mathrm{~b}$.

Ulva sp.

Chiang et al., 1974.

\section{CLADOPHORALES}

\section{Cladophoraceae}

Chaetomorpha aerea (Dillwyn) Kützing, 1849:379.

Ariga, 1921.

Chaetomorpha aerea f. versata Heydrich, 1894:273.

De Toni, 1895; Heydrich, 1894; Okamura, 1936; Shen and Fan, 1950.

Chaetomorpha basiretrorsa Setchell, 1926:72.

Chiang, 1960.

Chaetomorpha brachygona Harvey, 1858:87.

Chiang, 1960; Okamura, 1936; Shen and Fan, 1950; Yanrada, 1925a.

Chaetomorpha crassa (C. Agardh) Kützing, 1845:204.

Ariga, 1920; Chiang, 1960, 1973b; Fan, 1953a; Okamura, 1931, 1936; Shen and Fan, 1950; Taniguti, 1976;
Yamada, 1925a, 1950; Yoshikawa and Yoshikawa, 1977. Chaetomorpha linum (O.F. Müller) Kützing, 1 845:204.

Ariga, 1919; Chiang, 1960; Fan, 1953a; Horikawa, 1919;

Okamura, 1936; Shen and Fan, 1950; Tokida, 1954;

Yamada, 1925a, 1926, 1950; Yendo, 1916a.

Chaetomorpha spiralis Okamura, 1912:162.

Chiang, 1960, 1973b; Fan, 1953a.

Cladophora aokii Yamada, 1925a:85.

Okamura, 1936; Shen and Fan, 1950; Yamada, 1925a.

Cladophora fuliginosa Kützing, 1849:415.

Okamura, 1936; Tseng, 1983; Yamada, 1932b, 1934, 1950 .

Cladophora montagnei var. radicans Yamada, 1925a:87.

Okamura, 1936; Shen and Fan, 1950; Yamada, 1925a.

Cladophora patentiramea (Montagne) Kützing, 1849:416.

Okamura, 1936; Shen and Fan, 1950; Tseng, 1983; Yamada, $1925 a, 1950$.

Cladophora pellucida (Hudson) Kützing, 1845:208.

Okamura, 1936; Shen and Fan, 1950. As "C. prolifera":

Okamura, 1931.

Cladophora rugulosa Martens, 1866:112.

Ariga, 1920.

Cladophora scitula (Suhr) Kützing, 1849:399.

De Toni, 1895; Heydrich, 1894; Okamura, 1936; Shen and Fan, 1950.

Cladophora sibogae Reinbold, 1905:146.

Okamura, 1931, 1936; Shen and Fan, 1950; Taniguti, 1976; Yamada, 1950.

Cladophora sp.

Ariga, 1919, 1920; Fan, 1953a; Horikawa, 1919; Okamura, 1931; Shen and Fan, 1950; Tokida, 1939.

\section{CAULERPALES}

\section{Codiaceae}

Codium adhaerens (Cabrera) C. Agardh, 1823:457. Ariga, 1919, 1920; Chiang, 1960, 1962b; Horikawa, 1919; Okamura, 1936.

Codium arabicum Kützing, 1856:35.

Okamura, 1936; Shen and Fan, 1950; Tseng, 1983; Yamada, 1925a.

Codium contractum Kjellman, $1897 \mathrm{~b}: 35$. Ariga, 1920; Taniguti, 1976.

Codium cylindricum Holmes, 1896:250.

Taniguti, 1976.

Codium dichotomum (Hudson) S.F. Gray, $1821: 293$.

Fan, 1953a; Tokida, 1954. As "C. tomentosum": De Toni, 1895; Heydrich, 1894; Shen and Fan, 1950.

Codium formosanum Yamada, 1950:180.

Fan, 1953a; Yanıda, 1950; Yoshikawa and Yoshikawa, 1977.

Codium fragile (Suringar) Hariot, 1889:32.

As "C. mucronatum": Ariga, 1919; Horikawa, 1919. 
Codium intricatum Okamura, 1913:74.

Ariga, 1919, 1920; Chiang, 1962b, 1973b; Fan, 1953a; Higashi, 1934; Horikawa, 1919; Shen and Fan, 1950; Yamada, 1950.

Codium reediae Silva in Egerod, 1952:389.

Chiang, 1973a.

Codium repens (P. et H. Crouan) Vickers, 1905:56.

Fan, 1953a; Okamura, 1931, 1936; Shen and Fan, 1950;

Tseng, 1983; Yamada, 1925a.

Codium tenue Kützing, $1856: 33$.

Fan, 1953a; Okamura, 1936; Rho, 1958; Shen and Fan, 1950; Taniguti, 1976; Yamada, 1925a.

\section{UDOTEACEAE}

Chlorodesmis caespitosa J. Agardh, 1887:49.

Chiang, 1973b; Chihara, 1975; Yoshikawa and Yoshikawa, 1977. As "C. formosana”: Arasaki, 1964; Chihara, 1970; Okamura, 1930, 1936; Rho, 1958; Segawa, 1974; Shen and Fan, 1950; Yamada, 1925a.

Remarks: Ducker et al. (1965) and Ducker (1967) provide evidence for reducing $C$. formosana into synonomy with $C$. caespitosa (see also Fan, 1974).

Chlorodesmis comosa Bailey et Harvey in Harvey, 1858:29. Ariga, 1920; Higashi, 1934; Okamura, 1931, 1936; Rho, 1958; Shen and Fan, 1950; Yamada, 1925a.

Halimeda cuneata Hering in Krauss, 1846:214.

Higashi, 1934; Okamura, 1930, 1936; Shen and Fan, 1950; Taniguti, 1976; Yamada, 1925a, 1950.

Halimeda discoidea Decaisne, 1842:102. Ariga, 1920.

Halimeda incrassata var. ovata (J. Agardh) Barton, 1901:27. Okamura, 1936; Yendo, 1909.

Halimeda macroloba Decaisne, 1841:118.

Chiang, 1973a, 1973b; Okamura, 1915a, 1935b, 1936.

Halimeda opuntia (Linnaeus) Lamouroux, 1812:186.

Ariga, 1920; Chiang, 1973b; Su et al., 1982; Taniguti, 1976; Yamada, 1926.

Halimeda renschii Hauck, 1886:167.

As "H. opuntia f. renschii": Ariga, 1919, 1920; Chiang, 1962b; Horikawa, 1919; Okamura, 1915a, 1931, 1936;

Shen and Fan, 1950; Su et al., 1982; Yamada, 1925a, 1950.

Halimeda velasquezii W.R. Taylor, 1962:176.

As "H. opuntia f. intermedia" Chiang, 1962b.

\section{Caulerpaceae}

Caulerpa cupressoides var. lycopodium f. amicorum (Harvey) Weber-van Bosse, 1898:337.

Ariga, 1920.

Caulerpa freycinetti var. freycinetti $\mathrm{f}$. lata Weber-van Bosse, 1898:313.
Ariga, 1920; Okamura, 1936; Shen and Fan, 1950; Yoshikawa and Yoshikawa, 1977. As "C. freycinetti var. typica”: Okamura, 1931.

Caulerpa racemosa (Forsskål) J. Agardh, 1873:35.

Ariga, 1919; Fan, 1953a; Horikawa, 1919; Taniguti, 1976; Tokida, 1939.

Caulerpa racemosa var. clavifera f. macrophysa (Kützing) Weber-van Bosse, 1898:361.

Ariga, 1920; Chiang, 1962b; Okamura, 1936; Rho, 1958; Segawa, 1974; Yamada, 1925a, 1926, 1950. As "C. racemosa var. clavifera": Okamura, 1913, 1931; Shen and Fan, 1950; Yamada, 1925a, 1926; Tseng, 1983.

Caulerpa racemosa var. clavifera f. microphysa (Kützing) Weber-van Bosse, 1898:361.

Okamura, 1931, 1936; Yamada, 1950.

Caulerpa racemosa var. laetevirens (Montagne) Weber-van Bosse, 1898:366.

Chiang, 1960, 1973b; Okamura, 1930, 1931, 1936; Segawa, 1974; Shen and Fan, 1950; Taniguti, 1976; Yamada, 1925a, 1926, 1950; Yoshikawa and Yoshikawa, 1977.

Caulerpa racemosa var. occidentalis (J. Agardh) $\mathrm{B} \phi$ rgesen, $1907: 379$.

Yoshikawa and Yoshikawa, 1977.

Caulerpa racemosa var. peltata (Lamouroux) Eubank, 1946:421.

As "C. peltata": Chiang, 1960, 1962b; Okamura, 1931, 1932, 1936; Segawa, 1974; Shen and Fan, 1950; Tseng, 1936; Yamada, 1950.

Caulerpa racemosa var. turbinata (J. Agardh) Eubank, $1946: 420$.

As "C. racemosa var. chemnitzia": Chiang, 1960; Shen and Fan, 1950; Yamada, 1925a, 1926.

Caulerpa serrulata (Forsskål) C. Agardh, 1823:446.

Eubank, 1946; Segawa, 1974; Tseng, 1936; Chihara, 1975.

Caulerpa serrulata f. lata (Weber-van Bosse) Tseng 1936:178.

Tseng, 1983.

Caulerpa sertularioides (Gmelin) Howe, 1905:576.

Ariga, 1919; Chihara, 1975; Horikawa, 1919; Yamada, 1950.

Caulerpa sertularioides f. longipes (J. Agardh) Collins, 1909:415.

Ariga, 1920.

Caulerpa taxifolia (Vahl) C. Agardh, 1823:435.

Yamada, 1926.

Caulerpa webbiana Montagne, 1838:18.

Ariga, 1919 ; Horikawa, 1919.

Caulerpa webbiana f. disticha Weber-van Bosse, 1898:270.

Okamura, 1931, 1936; Shen and Fan, 1950.

Caulerpa webbiana f. tomentella (Harvey) Weber-van Bosse, 1898:270.

Ariga, 1920; Chihara, 1975; Okamura, 1931, 1936; Segawa, 1974; Shen and Fan, 1950; Tseng, 1936. 


\section{BRYOPSIDACEAE}

Bryopsis harveyana J. Agardh, 1887:22.

Okamura, 1931, 1936; Shen and Fan, 1950; Tseng, 1983; Yamada, 1925a, 1950.

Bryopsis indica A. et E.S. Gepp, 1908:169.

Chiang, 1960, 1962 b.

Bryopsis mucosa Lamouroux, $1809 \mathrm{~b}: 333$.

Chiang, 1960.

Bryopsis plumosa (Hudson) C. Agardh, $1823: 448$. Ariga, 1920; Chiang, 1960; De Toni, 1895; Heydrich, 1894; Okamura, 1936; Rho, 1958; Shen and Fan, 1950.

\section{DERBESIACEAE}

Derbesia lamourouxii (J. Agardh) Solier, 1847:162. Taniguti, 1976.

\section{SIPHONOCLADALES}

\section{SIPHONOCLADACEAE}

Boergesenia forbesii (Harvey) Feldmann, 1938:584. Chiang, 1962b, 1973b; Tseng, 1983; Yamada, 1950. As "Valonia forbesii": Ariga, 1919; Horikawa, 1919; Okamura, 1936; Shen and Fan, 1950; Yamada, 1925a.

\section{VALONIACEAE}

Boodlea coacta (Dickie) Murray et De Toni in Murray, 1889:245.

Yamada, 1926, 1950; Yoshikawa and Yoshikawa, 1977.

Boodlea composita (Harvey et J. Hooker) Brand, 1904:187. Chiang, 1960, 1973b; Okamura, 1936; Shen and Fan, 1950; Tseng, 1983; Yamada 1925a, 1950.

Boodlea montagnei (Harvey ex J.E. Gray) Egerod, 1952:332. As "B. paradoxa": Chiang, 1960; Okamura, 1936; Shen and Fan, 1950.

Boodlea siamensis Reinbold, 1901:191.

Chiang, 1962b; Okamura, 1931, 1936; Shen and Fan, 1950; Yamada, 1925a.

Boodlea van-bosseae Reinbold, 1905:148, 'van Bossei'.

Okamura, 1919, 1931, 1936; Shen and Fan, 1950. As "B. bosseae": Ariga, 1919; Horikawa, 1919.

Remarks: Following the examples given under Article 73.9 of the International Code of Botanical Nomenclature (Voss et al., 1983), "a hyphen is correctly used in an epithet after a word which could stand independently ...," we spell the species name "van-bosseae."

Chamaedoris orientalis Okamura et Higashi in Okamura, 1931:98.
Okamura, 1931, 1932, 1936; Shen and Fan, 1950.

Cladophoropsis herpestica (Montagne) Howe, 1914:31.

Shen and Fan, 1950.

Cladophoropsis sundanensis Reinbold, 1905:147.

Chiang, 1960; Su et al., 1982.

Cladophoropsis zollingeri (Kützing) Reinbold, 1905:147.

Chiang, 1960; Shen and Fan, 1950; Su et al., 1982;

Taniguti, 1976; Tseng, 1983. As "C. fasciculatus": Okamura, 1930, 1931; Tseng, 1983; Yamada, $1925 a$.

Remarks: There has been some question as to when and where the combination Cladophoropsis zollingeri was validly published. Most authors (e.g., Papenfuss, 1950; Chiang, 1962a; Taniguti, 1976) have accepted C. zollingeri (Kützing) B $\phi$ rgesen (1905:288) as correct. According to Article 33.1 of the International Code of Botanical Nomenclature (Voss et al., 1983), "the combination is not validly published unless the author definitely indicates that the epithet or epithets concerned are to be used in that particular combination." Parallel to the examples, such as Eulophus, cited in the Code, Borgesen (1905) has merely stated that "S. zollingeri" belongs here but did not actually make the combination in Cladophoropsis. The earliest valid combination we are aware of is: $C$. zollingeri (Kützing) Reinbold 1905:147.

Dictyosphaeria bokotensis Yamada, 1925a:81.

Okamura, 1936; Shen and Fan, 1950; Yamada, 1925a, 1950.

Dictyosphaeria cavernosa (Forsskål) B $\phi$ rgesen, 1932:2. Chiang, 1960, 1973b; Shen and Fan, 1950; Taniguti, 1976; Tseng, 1983. As "D. favulosa": Ariga, 1920; Okamura, 1930, 1931, 1936; Yamada, 1925a, 1926.

Microdictyon japonicum Setchell, 1925:107.

Chiang, 1962b; Tseng, 1983; Shen and Fan, 1950. As "Rhipidiphyllon reticulatum": Ariga, 1920; De Toni, 1895; Heydrich, 1894.

Remarks: Fan (1974:249-250,253-254) reviews taxonomic problems with the genus Rhipidiphyllon Heydrich, 1894:281. The type-locality for this genus is in east Taiwan.

Microdictyon nigrescens (Yamada) Setchell, 1925:107.

Okamura, 1936; Shen and Fan, 1950; Fan, 1974; Yamada, 1950. As "Rhipidiphyllon nigrescens": Yamada, $1925 a$.

Microdictyon okamurae Setchell, 1925:107, 'okamurai'. Okamura, 1931; Shen and Fan, 1950; Tseng; 1983.

Remarks: The correct spelling for the Latinization of Professor Okamura's name is "okamurae" and has been corrected throughout this study.

Struvea anastomosans (Harvey) Piccone et Grunow ex Piccone, 1884:20.

Ariga, 1920; Tseng, 1983.

Struvea delicatula Kützing, 1866:1.

Chiang, 1960; De Toni, 1895; Heydrich, 1894; Oka- 
mura, 1930, 1931, 1936; Shen and Fan, 1950; Yamada, $1925 \mathrm{~b}, 1926$.

Valonia aegagropila C. Agardh, 1823:429.

Chiang, 1973b; Chihara, 1975; Shen and Fan, 1950; Tseng, 1983; Yamada, 1925a, 1950; Yoshikawa and Yoshikawa, 1977.

Valonia fastigiata Harvey in J. Agardh, 1887:101.

Okamura, 1931; Shen and Fan, 1950.

Valonia utricularis (Roth) C. Agardh, 1823:431.

Okamura, 1931, 1936; Shen and Fan, 1950; Tseng, 1983; Yamada, 1926; Yendo, 1914.

Valonia verticillata Kützing, $1849: 508$.

Okamura, 1930.

Valoniopsis pachynema (Martens) B $\phi$ rgesen, 1934:10.

Chiang, 1960, 1973b; Shen and Fan, 1950; Tseng, 1983; Yamada, 1950; Yoshikawa and Yoshikawa, 1977. As "Valonia confervoides”: Ariga, 1920; Okamura, 1909, 1931, 1936; Yamada, 1925a.

\section{AnADYOMENACEA}

Anadyomene wrightii Harvey in Gray, $1866: 48$.

Ariga, 1920; Chiang, 1973b; Okamura, 1936; Shen and Fan, 1950; Tseng, 1983; Yamada, 1925a, 1926, 1950.

\section{DASYCLADALES}

\section{Acetabulariaceae}

Acetabularia gigas Solms-Laubach, 1895:23.

Okamura, 1936; Shen and Fan, 1950; Yamada, 1925a. Acetabularia major Martens, 1866:25.

Okamura, 1936; Shen and Fan, 1950; Yamada, 1925a.

Bornetella sphaerica (Zanardini) Solms-Laubach, 1893:92.

Chiang, 1973b.

Neomeris annulata Dickie, 1874:198.

Chiang, 1973b.

\section{PHAEOPHYTA}

\section{ECTOCARPALES}

\section{ECTOCARPACEAE}

Ectocarpus breviarticulatus J. Agardh, 1847:7.

Okamura, 1936; Segawa, 1974; Shen and Fan, 1950;

Tseng, 1983; Yamada, 1925b, 1950.

Ectocarpus laurenciae Yamada, 1931 a:66.

Okamura, 1936; Shen and Fan, 1950; Yamada, 193 la.

Ectocarpus spinosus Kützing, 1843:288.

Heydrich, 1894; Shen and Fan, 1950.

Ectocarpus van-bossea Setchell et Gardner in Setchell, 1924:170.

Yamada, 1950.

Ectocarpus sp.

Chiang, 1976.

Feldmannia formosana (Yamada) Itono, 1973:162.

As "Ectocarpus formosanus": Yamada, 1950.

Giffordia mitchelliae (Harvey) Hamel, 1939:xiv.

As "Ectocarpus mitchelliae": Chiang, 1960; Okamura, 1936; Shen and Fan, 1950; Tseng, 1983; Yamada, $1925 \mathrm{~b}$.

\section{CHORDARIALES}

\section{Corynothlaeaceae}

Leathesia difformis (Linnaeus) Areschoug, 1847:376.

Taniguti, 1976.

\section{SPHACELARIALES}

\section{Sphacelariaceae}

Sphacelaria furcigera var. tenuis Yamada, 1941:196.

Shen and Fan, 1950; Yamada, 1941.

Sphacelaria tribuloides Meneghini, 1840:2.

Chihara, 1975; De Toni, 1895; Heydrich, 1894; Okamura, 1897, 1936; Segawa, 1974; Shen and Fan, 1950; Tseng, 1983; Yamada, 1925b.

\section{DICTYOTALES}

\section{Dictyotaceae}

Dictyopteris delicatula Lamouroux, $1809 \mathrm{~b}: 332$. Yamada, 1950.

Dictyopteris repens (Okamura) B $\phi$ rgesen, 1920a:265. "Tseng, 1983; Yoshikawa and Yoshikawa, 1977. As "Neurocarpus repens": Okamura, 1931, 1936; Shen and Fan, 1950.

Dictyopteris undulata Holmes, 1896:251.

Tseng, 1983. As "Haliseris undulata": Okamura, 1907. As "Neurocarpus undulata": Higashi, 1934; Okamura, 1930, 1936; Shen and Fan, 1950. As "N. undulata f. plana”: Chiang, 1960; Okamura, 1930, 1936.

Dictyota bartayresii Lamouroux, $1809 \mathrm{~b}: 331$.

Okamura, 1931, 1936; Shen and Fan, 1950. 
Dictyota ceylanica var. anastomosans Yamada, 1950:186.

Yamada, 1950; Yoshikawa and Yoshikawa, 1977.

Dictyota dichotoma (Hudson) Lamouroux, 1809a:42.

Ariga, 1919; Higashi, 1934; Horikawa, 1919; Okamura, 1930; Rho, 1958; Shen and Fan, 1950; Tokida, 1954;

Yamada, 1925b.

Dictyota dilata Yamada, 1925b:252

Okamura, 1936; Segawa, 1974; Shen and Fan, 1950; Yamada, 1925b.

Dictyota divaricata Lamouroux, 1809a:43.

Chiang, 1960; Okamura, 1930; Shen and Fan, 1950;

Yamada, 1925b.

Dictyota hamifera Setchell, 1926:92.

Yamada, 1950.

Dictyota linearis (C. Agardh) Greville, 1830:xliii.

Ariga, 1919, 1920; Horikawa, 1919.

Dictyota patens J. Agardh, 1882:93.

Taniguti, 1976.

Remarks: Howe (1920) and Allender and Kraft (1983)

consider this taxon to be a synonym of $D$. bartayresii Lamouroux.

Dictyota spinulosa Hooker et Arnott, 1838:275.

Ariga, 1920.

Dilophus okamurae Dawson, 1950:83, 'okamurai'.

Chilhara, 1970, 1975; Fan, 1953b; Segawa, 1974; Tseng, 1983. As "D. marginatus": Chiang, 1960; Okamura, 1936; Shen and Fan, 1950.

Dilophus radicans Okamura, 1916:7.

Chiang, 1960; Okamura, 1936; Shen and Fan, 1950.

Remarks: Fan (1953b) considers this name to be in need of synonomy since he believes $D$. radicans may be the prostrate form of Padina commersonii (see also Lewis et al., 1987).

Lobophora variegata (Lamouroux) Womersley, 1967:221.

Tseng, 1983. As "Gymnosorus collaris": Ariga, 1920. As

"Zonaria variegata”: Heydrich, 1894.

Pachydictyon coriaceum (Holmes) Okamura, 1899:39.

Ariga, 1920; Chiang, 1976; Chihara, 1970; Okamura,

1936; Rho, 1958; Shen and Fan, 1950.

Padina arborescens Holmes, 1896:251.

Higashi, 1934.

Padina australis Hauck, 1887:44.

Chiang, 1960, 1962b; Okamura, 1931, 1932, 1936; Rho, 1958; Shen and Fan, 1950; Su et al., 1982; Tseng, 1936, 1983; Yamada, 1925b, 193 la, 1950.

Padina boryana Thivy in Taylor, 1966:355.

Tseng, 1983.

Remarks: Papenfuss (1977) considered both P. tenuis (C. Agardh) Bory and P. commersonii Bory to be synonyms of Lobophora variegata (Lamouroux) Womersley, and recognized $P$. boryana Thivy as the name for this taxon. Tseng (1983) apparently follows this, listing $P$. commersonii as a synonym of $P$. boryana, whereas Womersley and Bailey (1970) and Allender and Kraft (1983) considered $P$. commersonii and $P$. boryana to be synonyms of $P$. tenuis.
Padina crassa Yamada, 193 la:67.

Ariga, 1920; Chiang, 1960; Yoshikawa and Yoshikawa, 1977.

Padina durvillaei Bory, 1827:591.

Ariga, 1920; Chiang, 1973a; De Toni, 1895; Heydrich, 1894; Okamura, 1935b; Shen and Fan, 1950.

Padina japonica Yamada, 193 la:69.

As "P. pavonia": Ariga, 1919; De Toni, 1895; Heydrich, 1894 ; Horikawa, 1919.

Padina minor Yamada, 1925b:251.

Chiang, 1960, 1962a, 1962b, 1973b, 1976; Chihara, 1975; Okamura, 1931, 1932, 1936; Rho, 1958; Shen and Fan, 1950; Taniguti, 1976; Tseng, 1983; Yamada, 1925b, 193 la, 1950; Yoshikawa and Yoshikawa, 1977.

Padina tenuis Bory, 1827:590.

As "Padina commersonii": Okamura, 1931, 1936; Shen and Fan, 1950; Yamada, 1925b, 1931 a.

Remarks: See "Remarks" herein under Padina boryana Thivy in Taylor.

Spatoglossum pacificum Yendo, 1920:2.

Chiang, 1973a.

Zonaria coriacea Yamada, 1925b:249.

Chiang, 1960; Okamura, 1936; Shen and Fan, 1950; Yamada, 1925b.

Zonaria diesingiana J. Agardh, 1841:443.

Ariga, 1920; Chiang, 1960; Okamura, 1930; Shen and Fan, 1950; Tseng, 1983; Yamada, 1925b.

Zonaria flabellata (Okamura) Papenfuss, 1944:341.

As "Homoeostrichus flabellatus": Taniguti, 1976.

Zonaria harveyana (Kützing) Areschoug, 1851:26.

As "Homoeostrichus multifidus": Chiang, 1960; Okamura, 1936; Shen and Fan, 1950; Yamada, 1925b.

Zonaria nigrescens Sonder, 1845:50.

Heydrich, 1894; Shen and Fan, 1950.

Zonaria stipitata Tanaka et Nozawa, 1962:183.

Chiang and Chou, 1980.

Zonaria sp.

Su et al., 1982.

\section{SCYTOSIPHONALES}

\section{ISHIGEACEAE}

Ishige okamurae Yendo, 1907:154, 'okamurai'. Chiang, 1960, 1973b; Higashi, 1934; Okamura, 1936; Rho, 1958; Shen and Fan, 1950; Yamada, 1925b.

Ishige sinicola (Setchell et Gardner) Chihara, 1969:3. As "I. foliacea": Rho, 1958; Tseng, 1936.

\section{Punctariaceae}

Petalonia fascia (O.F. Müller) Kuntze, 1898:419. As "Ilea fascia": Chiang, 1960, 1976; De Toni, 1895; Rho, 1958; Shen and Fan, 1950; Tokida, 1954; Yamada, 1925b. As "Phyllitis fascia": Heydrich, 1894. 


\section{SCYTOSIPHONACEAE}

Chnoospora implexa (Hering) J. Agardh, 1848:172. Chiang, 1960; Okamura, 1931, 1936; Shen and Fan, 1950; Taniguti, 1976; Tokida, 1939; Tseng, 1983; Yamada, 1925b, 1950.

Chnoospora minima (Hering) Papenfuss, 1956:69.

Chiang, 1976; Segawa, 1974. As "Chnoospora pacifica": Okamura, 1931, 1936; Shen and Fan, 1950; Tokida, 1939; Yamada, 1950.

Colpomenia sinuosa (Mertens et Roth) Derbès et Solier in Castagne, 1851:95.

Ariga, 1919, 1920; Chiang, 1960, 1976; De Toni, 1895; Heydrich, 1894; Horikawa, 1919; Okamura, 1930; Shen and Fan, 1950; Taniguti, 1976; Tokida, 1954; Yamada, 1925b, 1950; Yoshikawa and Yoshikawa, 1977.

Endarachne binghamiae J. Agardh, 1896:27.

Ariga, 1919, 1920; Chiang, 1960, 1973b, 1976; Cotton, 1915; Fan, 1953a; Higashi, 1934; Horikawa, 1919; Okamura, 1930, 1936; Rho, 1958; Shen and Fan, 1950; Taniguti, 1976; Tilden, 1929; Tseng, 1983; Yamada, 1925b.

Hydroclathrus clathratus (Bory) Howe, 1920:590.

Chiang, 1960, 1962a, 1973b; Okamura, 1936; Rho, 1958; Shen and Fan, 1950; Taniguti, 1976; Tseng, 1983; Yamada, 1950; Yoshikawa and Yoshikawa, 1977. As " $H$. cancellatus": De Toni, 1895; Heydrich, 1894; Higashi, 1934; Okamura, 1897, 1931; Yamada, 1925b.

Rosenvingea orientalis (J. Agardh) B $\phi$ rgesen, 1914:26.

Okamura, 1936; Shen and Fan, 1950; Tseng, 1983. As "Hydroclathrus orientalis": De Toni, 1895; Heydrich, 1894.

Scytosiphon lomentaria (Lyngbye) J. Agardh, 1848:126, 'lomentarium'.

Chiang, 1973a, 1976; Okamura, 1935b, 1936; Segawa, 1974; Tokida, 1954; Yamada, 1925b, 1928. As "S. lomentarius": Chihara, 1970, 1975.

\section{FUCALES}

\section{Cystoseiraceae}

Cystoseira prolifera J. Agardh, 1848:215. Chiang, 1976.

Cystoseira sp. Nizamuddin, 1970.

Hormophysa triquetra (Linnaeus) Kützing, 1843:359. Chiang, 1973a; Okamura, 1935b; Su et al., 1982.

\section{SARGaSSACEAE}

Sargassum amabile Yamada, 1944a:1. Shen and Fan, 1950.

Sargassum aquifolium (Turner) C. Agardh, 1820:12. Okamura, 1931, 1936; Shen and Fan, 1950.

Sargassum baccularia (Mertens) C. Agardh, 1824:304.
Chou and Chiang, 1981.

Sargassum berberifolium J. Agardh, 1848:337.

Okamura, 1936; Shen and Fan, 1950; Yamada, 1925b.

Sargassum binderi Sonder in J. Agardh, 1848:328.

Chiang, 1976; Chou and Chiang, 1981; Okamura, 1936;

Shen and Fan, 1950; Yamada, 1925b.

Sargassum carpophyllum J. Agardh, 1848:304.

Chou and Chiang, 1981; Yamada, 1942.

Sargassum coriifolium J. Agardh, 1889:86.

Okamura, 1936; Shen and Fan, 1950; Yamada, 1925b.

Sargassum coriifolium f. duplicatum Yamada, 1950:192.

Yamada, 1950; Yoshikawa and Yoshikawa, 1977.

Sargassum coriifolium f. prolongatum (Okamura) Yamada, 1950: 192.

Yamada, 1950. As "S. prolongatum": Okamura, 1931, 1932, 1936; Shen and Fan, 1950.

Sargassum crassifolium J. Agardh, 1848:326.

Chihara, 1975; Chou and Chiang, 1981; Tseng, 1983;

Yamada, 1942, 1950; Yoshikawa and Yoshikawa, 1977.

Sargassum crispifolium Yamada, 1931a:72.

Chiang, 1960, 1962b.

Sargassum cristaefolium C. Agardh, 1820:13.

Ariga, 1920; Chiang, 1976; Chou and Chiang, 1981;

Cotton, 1915; Okamura, 1931, 1935b, 1936; Shen and

Fan, 1950; Tilden, 1929. As "S. biserrula": De Toni, 1895; Heydrich, 1894.

Sargassum duplicatum J. Agardh, 1889:90.

Ariga, 1921; Chiang, 1976; Chou and Chiang, 1981;

Liu, 1982; Okamura, 1936; Shen and Fan, 1950; Taniguti, 1976; Yamada, 1925b.

Sargassum echinocarpum J. Agardh, 1848:327.

Yamada, 1950.

Sargassum fulvellum (Turner) C. Agardh, 1820:34.

Ariga, 1920.

Sargassum glaucescens J. Agardh, 1848:306.

Chiang, 1973a; Chou and Chiang, 1981; Okamura, 1935b.

Sargassum hemiphyllum (Turner) C. Agardh, 1820:39.

Ariga, 1920; Chiang, 1976; Chou and Chiang, 1981;

Tilden, 1929; Tseng et al., 1985.

Sargassum henslowianum C. Agardh in J. Agardh, 1848:315. Chou and Chiang, 1981.

Sargassum heterocystum Montagne, 1842:250.

Chou and Chiang, 1981.

Sargassum horneri (Turner) C. Agardh, 1820:38.

Ariga, 1919; Chiang, 1960; Chou and Chiang, 1981;

Horikawa, 1919; Okamura, 1936; Rho, 1958; Shen and

Fan, 1950; Tokida, 1954; Tseng et al., 1962, 1985;

Yamada, 1925b.

Sargassum ilicifolium (Turner) C. Agardh, 1820:11.

Ariga, 1920; Chou and Chiang, 1981; Okamura, 1936;

Shen and Fan, 1950; Tseng, 1983; Yamada, 1925b.

Sargassum ilicifolium var. conduplicatum Grunow in Webervan Bosse, 1913a:160.

Yamada, 1942. 
Sargassum kasyotense Yamada, 1942:553.

Shen and Fan, 1950; Yamada, 1942.

Sargassum kuetzingii Setchell, 1931:249.

Chou and Chiang, 1981.

Sargassum kushimotense Yendo, 1905:157.

Chou and Chiang, 1981.

Sargassum muriocystum J. Agardh, 1848:314.

Ariga, 1920; De Toni, 1895; Okamura, 1895, 1936;

Shen and Fan, 1950.

Sargassum plagiophyllum C. Agardh, 1824:309.

Yamada, 1942.

Sargassum polycystum C. Agardh, 1824:304.

Ariga, 1920; Chou and Chiang, 1981; Okamura, 1931, 1936; Shen and Fan, 1950, Su et al., 1982; Tseng, 1983; Yamada, 1942, 1950; Yoshikawa and Yoshikawa, 1977. As "S. microphyllum": Chiang, 1976; Yamada, 1925 b.

Sargassum rostratum J. Agardh, 1896:55.

Chou and Chiang, 1981; Okamura, 1936; Shen and Fan, 1950; Tseng et al., 1985.

Sargassum sandei Reinbold in Weber-van Bosse, 1913a:158. Chiang, 1960, 1976; Chou and Chiang, 1981; Okamura, 1936; Segawa, 1974; Yamada, 1925b; Yoshikawa and Yoshikawa, 1977.

Sargassum sandei f. heterophyllum Yamada, 1950:192.

Yamada, 1950.

Sargassum serratifolium C. Agardh, 1820:16.

Chiang, 1973a.

Sargassum siliquosum J. Agardh, 1848:316.

Chiang, 1976; Chou and Chiang, 1981; Okamura, 1931, 1936; Rho, 1958; Shen and Fan, 1950; Yamada, 1925b, 1942.
Sargassum swartzii (Turner) C. Agardh, 1820:11.

Yamada, 1942.

Sargassum telephifolium (Turner) C. Agardh in J. Agardh, 1889:107.

Okamura, 1931, 1936; Shen and Fan, 1950.

Sargassum tenuifolium Yamada, 1942:505.

Yamada, 1942.

Sargassum vulgare C. Agardh, 1820:3.

Ariga, 1919; Horikawa, 1919.

Sargassum sp.

Chiang, 1962b, 1976; Chiang et al., 1974; Chou and Chiang, 1981; Su et al., 1982.

Turbinaria conoides (J. Agardh) Kützing, 1860:24.

Chiang, 1973b; Okamura, 1931, 1936; Shen and Fan, 1950; Tseng, 1983.

Turbinaria filamentosa Yamada, 1925b:243.

Chiang, 1960, 1976; Fan, 1974; Shen and Fan, 1950; Yamada, 1925b. As “T. filiformis": Okamura, 1936; Yoshikawa and Yoshikawa, 1977.

Remarks: Fan (1974:252, 254) suggests that the characteristic of this species (i.e., elongate filiform receptacle) may not reflect a species difference, but rather reflect an ecological or physiological state.

Turbinaria ornata (Turner) J. Agardh, 1848:266.

Chiang, 1960, 1962b, 1976; De Toni, 1895; Okamura, 1895, 1931, 1936; Shen and Fan, 1950; Su et al., 1982; Yamada, 1925b, 1950.

Turbinaria trialata (J. Agardh) Kützing, 1860:24.

Chiang, 1962b; Yamada, 1950.

Turbinaria sp.

Ariga, 1919; Horikawa, 1919.

\section{RHODOPHYTA}

\section{GONIOTRICHALES}

\section{GoniotrichaceaE}

Chroodactylon ornatum (C. Agardh) Basson, 1979:67. As "Asterocytis ornata": Shen and Fan, 1950; Tanaka, 1944, 1952. As "Asterocytis ornata f. simplex": Shen and Fan, 1950; Tanaka, 1944, 1952. As "Asterocytis ramosa $\mathrm{f}$. simplex": Tanaka, 1944.

Stylonema alsidii (Zanardini) Drew, 1956:72.

As "Goniotrichum alsidii": Tanaka, 1952.

Remarks: See Wynne (1985) for discussion of use of the generic name Stylonema Reinsche, and Drew and Ross (1965) on use of some generic names in the Bangiophycidae.

\section{BANGIALES}

\section{ERYTHROPELTIDACEAE}

Erythrotrichia biseriata Tanaka, 1944:86. Shen and Fan, 1950; Tanaka, 1944, 1951, 1952.
Erythrotrichia carnea f. tenuis Tanaka, 1944:92. Shen and Fan, 1950; Tanaka, 1944.

\section{BANGIACEAE}

Bangia atropurpurea (Roth) C. Agardh, 1824:76. Ariga, 1920. As "B. fuscopurpurea": Chiang, 1962a, 1973b; Fan, 1953a; Okamura, 1936; Rho, 1958; Sheath and Cole, 1984; Shen and Fan, 1950; Tanaka, 1950, 1952; Taniguti, 1976; Tokida, 1954.

Bangia yamadae Tanaka, 1944:84, 'yamadai'. Shen and Fan, 1950; Tanaka, 1944, 1950, 1952.

Bangia sp. Chiang, 1973b.

Porphyra angusta Okamura et Ueda in Ueda, 1932:28. Liaw and Chiang, 1979; Wang and Chiang, 1977.

Porphyra crispata Kjellman, 1897a:15. Ariga, 1920; Chiang, 1962a; Chihara, 1975; Dawson, 1954c; Fan, 1953a; Okamura, 1931, 1936; Shen and Fan, 1950; Tanaka, 1952; Taniguti, 1976; Wang and Chiang, 1977; Ueda, 1932. 
Porphyra dentata Kjellman, 1897a:13.

Wang and Chiang, 1977.

Porphyra suborbiculata Kjellman, 1897a:10.

Okamura, 1930, 1936; Wang and Chiang, 1977.

Porphyra sp.

Wang and Chiang, 1977.

\section{NEMALIALES}

Remarks: For discussion on the correct spelling of the name of this order see Nicolson and Norris, 1983.

\section{ACROCHAETIACEAE}

Liagorophila endophytica Yamada, 1944b:16.

Fan, 1974; Shen and Fan, 1950; Yamada, 1944b. As "Liagora orientalis": Yamada, 1938a.

\section{Dermonemat ACEAE}

Dermonema frappieri (Montagne et Millardet) B $\phi$ rgesen, 1942:42.

Chiang, 1969, 1971, 1973b; Chihara, 1975; Segawa, 1974; Tseng, 1983.

Dermonema gracile Martens ex Weber-van Bosse, 1921:204. Fan, 1953a; Okamura, 1931, 1936; Shen and Fan, 1950; Tokida, 1939; Tseng, 1945. As “D. dichotomum”: Ariga, 1920; Chen and Chiang, 1982; De Toni, 1895; Fan, 1974; Heydrich, 1894.

Dermonema pulvinata (Grunow in Holmes) Fan, 1962:337. Chen and Chiang, 1982; Chihara, 1975; Fan, 1953a; Tseng, 1983; Umezaki, 1972. As "Nemalion pulvinatum": Chiang, 1962a; Chihara, 1970; Okamura, 1931, 1936; Rho, 1958; Segawa, 1974; Shen and Fan, 1950; Tokida, 1939.

Remarks: Papenfuss (1967:96-97) points out that Dermonema Heydrich had been a nomen nudum until Heydrich (1894:289) published $D$. dichotomum. The typelocality for the genus is therefore Taiwan (see also Fan 1974:249, 253).

Dotyophycus yamadae (Ohmi et Itono) Abbott et Yoshizaki, $1981: 225$.

Chiang and Chen, 1983.

Liagoropsis schrammi (P. et H. Crouan in Mazè et Schramm) Doty and Abbott, 1964:443.

Fan, 1974. As "L. maxima": Shen and Fan, 1950; Yamada, $1944 c$.

Yamadaella cenomyce (Decaisne) Abbott, 1970:117.

Chiang, 1973b. As "L. annulata”: Okamura, 1931, 1936. As "L. caenomyce": Chihara, 1975; Segawa, 1974; Shen and Fan, 1950; Tseng, 1941a; Yamada, 1938a.

\section{LIAGORACEAE}

Helminthocladia australis Harvey, 1863, pl. 272.

Chen et al., 1981.
Liagora boergesenii Yamada, 1938a:11

Chiang, 1971, 1972; Chiang and Chen, 1982; Chihara, 1975; Shen and Fan, 1950; Yamada, 1938a.

Liagora ceranoides Lamouroux, 1816:239.

Chiang and Chen, 1982.

Liagora ceranoides var. leprosa (J. Agardh) Yamada, 1938a:21.

Segawa, 1974; Shen and Fan, 1950; Yamada, 1938a.

Liagora ceranoides var. pulverulenta (C. Agardh) Yamada, 1938a:21.

Shen and Fan, 1950; Yamada, 1938a.

Liagora decussata Montagne, 1849:64.

Chiang and Chen, 1982; Shen and Fan, 1950; Yamada, 1938 a.

Liagora farinosa Lamouroux, $1816: 240$.

Chihara, 1975; Shen and Fan, 1950; Tseng, 1941a; Yamada, 1938a.

Remarks: Recently Fan and Wang (1974:492) proposed a new generic name, Ganonema, for this taxon. However, we follow the opinion of Abbott (1976:130), and for the time being continue to recognize this taxon as a Liagora (see also Norris and Bucher, 1982:190).

Liagora orientalis J. Agardh, 1896:99.

Ariga, 1920; Chiang and Chen, 1982; Fan, 1974; Okamura, 1936; Shen and Fan, 1950; Yamada, 1932b. As "L. formosana": Yamada, 1938a.

Remarks: Yamada (1944b:18-19) reduced his species L. formosana (1938a:32) into synonomy with L. orientalis.

Liagora rugosa Zanardini, 1851:36.

Ariga, 1920.

Liagora segawai Yamada, 1938a:1.

Chiang and Chen, 1982.

Liagora setchellii Yamada, 1938a:13.

Chiang and Chen, 1982; Shen and Fan, 1950; Tseng, 1941 a; Yamada, 1938a; Yoshikawa and Yoshikawa, 1977.

Liagora valida Harvey, 1853:138.

Chiang and Chen, 1982; Okamura, 1931, 1936; Shen and Fan, 1950.

\section{Chaetangiaceae}

Actinotrichia fragilis (Forsskål) $\mathrm{B} \phi$ rgesen, 1932:6. Chihara, 1970, 1975; Shen and Fan, 1950; Tseng, 1941 b. As “Actinotrichia rigida": Ariga, 1920.

Galaxaura arborea Kjellman, 1900:72.

Ariga, 1920; Chiang, 1962a; Yendo, 1918; Yoshikawa and Yoshikawa, 1977.

Remarks: The taxonomy and nomenclature of this genus have been confused by morphological plasticity and uncertain life histories. Since the hypothesis of an alternation of morphologically different sexual and tetrasporic forms of Galaxaura (Howe 1917, 1918), there has been much speculation as to which tetrasporic and sexual "species" represent stages within the same life history (e.g., B $\phi$ rgesen, 1920b; Chou, 1945, 1947; Dawson, 1953; 
Taylor, 1960). We hold the opinion that until culture studies conclusively link the different reproductive stages into the life history, each reproductive form should be recognized by its previous species name.

Galaxaura canaliculata Kützing, 1849:530.

Heydrich, 1894; Shen and Fan, 1950.

Galaxaura clavigera Kjellman, 1900:76.

Chiang, 1962b; ltono, 1977a; Okamura, 1931, 1936;

Shen and Fan, 1950; Tanaka, 1936.

Remarks: Papenfuss, Mshigeni, and Chiang (1982:411) consider this species to be a synonym of Galaxaura marginata (Ellis et Solander) Lamouroux.

Galaxaura distenta Harvey, 1859:331.

Ariga, 1920.

Galaxaura elegans Tanaka, 1935:52.

Okamura, 1936; Shen and Fan, 1950; Tanaka, 1935, 1936.

Galaxaura elongata J. Agardh, 1876:529.

Itono, 1977a; Okamura, 1936; Shen and Fan, 1950;

Tanaka, 1936; Tseng, 1941 b; Yendo, $1916 \mathrm{~b}$; Yoshikawa and Yoshikawa, 1977.

Remarks: Considered a synonym of $G$. rugosa (Ellis et Solander) Lamouroux (1816:263) by Papenfuss, Mshigeni, and Chiang (1982).

Galaxaura falcata Kjellman, 1900:73.

Ariga, 1919; Horikawa, 1919, Taniguti, 1976.

Galaxaura fasciculata Kjellman, 1900:53.

ltono, 1977a; Okamura, 1936; Shen and Fan, 1950;

Tanaka, 1936; Tseng, $194 \mathrm{lb}$.

Galaxaura fastigiata Decaisne 1842:116.

Ariga, 1920; Chiang, 1962a, 1962b; Chihara, 1970,

1975; 1tono, 1977a; Okamura, 1931, 1936; Segawa,

1974; Shen and Fan, 1950; Tanaka, 1936; Tanaguti,

1976; Yoshikawa and Yoshikawa, 1977.

Remarks: Considered a synonym of $G$. oblongata (Ellis et Solander) Lamouroux (1816: 262) by Papenfuss, Mshigeni, and Chiang (1982).

Galaxaura filamentosa Chou in Taylor, 1945:139.

ltono, 1977a.

Galaxaura lapidescens (Ellis et Solander) Lamouroux, 1816:264.

De Toni, 1895.

Galaxaura lapidescens f. villosa J. Agardh, 1876:530.

Heydrich, 1894; Okamura, 1897; Shen and Fan, 1950.

Galaxaura latifolia Tanaka, 1935:54.

Okamura, 1935a, 1936; Shen and Fan, 1950; Tanaka, 1935, 1936.

Galaxaura oblongata (Ellis et Solander) Lamouroux, 1816:262.

Chiang, 1973a, 1973b; Okamura, 1935b; Su et al., 1982.

Galaxaura obtusata (Ellis et Solander) Lamouroux, 1816:262.

Itono, 1977a; Okamura, 1936; Papenfuss, Mshigeni, and
Chiang, 1982; Shen and Fan, 1950; Su et al., 1982;

Tanaka, 1936; Tseng, $194 \mathrm{lb}$.

Galaxaura pacifica Tanaka, 1935:55.

Itono, 1977a; Okamura, 1935a, 1936; Shen and Fan, 1950; Tanaka, 1935, 1936.

Remarks: Considered a synonym of $G$. rugosa (Ellis et Solander) Lamouroux (1816:262) by Papenfuss, Mshigeni, and Chiang (1982).

Galaxaura robusta Kjellman, 1900:85.

Chihara, 1970, 1975; Okamura, 1936; Segawa, 1974;

Shen and Fan, 1950; Tanaka, 1936.

Remarks: Considered a synonym of $G$. obtusata (Ellis et

Solander) Lamouroux by Papenfuss, Mshigeni, and Chiang (1982).

Galaxaura rudis Kjellman, 1900:43.

Okamura, 1931, 1936; Shen and Fan, 1950; Tanaka, 1936; Tseng, $1941 \mathrm{~b}$.

Remarks: Considered a synonym of G. lapidescens (Ellis et Solander) Lamouroux by Papenfuss, Mshigeni, and Chiang (1982).

Galaxaura marginata (Ellis et Solander) Lamouroux, 1816:264.

Papenfuss, Mshigeni, and Chiang, 1982. As "Galaxaura tenera": Chiang, 1973a, 1973b; Okamura, 1935a; Su et al., 1982.

Remarks: Material from Taiwan determined as G. tenera was re-identified by Papenfuss, Mshigeni, and Chiang (1982) to be this species.

Galaxaura veprecula Kjellman, 1900:80.

Ariga, 1920; Chihara, 1975; Itono, 1977a; Okamura,

1936; Rho, 1958; Shen and Fan, 1950; Tanaka, 1936;

Tseng, 1983; Yendo, 1918.

Remarks: Papenfuss, Mshigeni, and Chiang (1982) consider this species to be a synonym of $G$. marginata (Ellis et Solander) Lamouroux.

Scinaia boergesenii Tseng, 1941:100.

Shen and Fan, 1950.

Scinaia cottonii Setchell, 1914:103.

Shen and Fan, 1950.

Scinaia moniliformis J. Agardh, 1885:72.

Chiang, 1962a; Yoshikawa and Yoshikawa, 1977.

Scinaia pseudojaponica Yamada et Tanaka in Yamada, 1938b:127.

Chiang, 1962a, 1970, 1973b; Shen and Fan, 1950.

\section{BONNEMAISONIACEAE}

Asparagopsis taxiformis (Delile) Trevisan, 1845:45. As "A. sanfordiana": Ariga, 1919; Fan, 1953a; Horikawa, 1919; Okamura, 1931, 1936; Shen and Fan, 1950.

Delisea fimbriata (Lamouroux) Montagne, 1844:155.

Chihara, 1970, 1975. As "D. japonica": Cotton, 1915; Tilden, 1929. 


\section{GELIDIALES}

\section{Gelidiacea e}

Gelidium amansii Lamouroux in Kützing 1868:16. Cotton, 1915; Fan, 1953a; Okamura, 1913, 1915b, 1930; Oshima, 1915; Rho, 1958; Santelices and Stewart, 1985; Tilden, 1929; Tokida, 1954.

Gelidium amansii f. elegans Okamura, 1934a:56. Chiang, 1962a; Fan, 1951.

Gelidium amansii f. latioris Okamura, 1935b:443. Chiang, 1962a; Fan, 1951; Okamura, 1935b, 1936; Shen and Fan, 1950.

Gelidium "cartilagineum". Ariga, 1919; Horikawa, 1919.

Remarks: As with all taxa in this paper, we are reporting the name used by the author in recording the taxon from Taiwan. We recognize that Dixon (1967) has shown the basionym Fucus cartilagineus Linnaeus (1753) to be correctly Plocamium cartilagineum (Linnaeus) Dixon. However, in this case, we suggest the material of Ariga (1919) and Horikawa (1919) probably belongs to Gelidium, rather than Plocamium, but cannot be sure what species it is.

Gelidium crinale (Turner) Lamouroux in Bory, 1825:191. Ariga, 1920.

Gelidium divaricatum Martens, 1866:30.

Okamura, 1934a, 1936; Taniguti, 1976.

Gelidium japonicum (Harvey) Okamura, 1901:57.

Arasaki, 1964; Ariga, 1919, 1920; Chiang, 1962a; Fan, 1951, 1953a; Higashi, 1934; Horikawa, 1919; Okamura, 1901, 1915b, 1930, 1934a, 1936; Oshima, 1915; Santelices and Stewart, 1985; Shen and Fan, 1950.

Gelidium kintaroi (Okamura) Yamada, 1941:201. Fan, 1951, 1953a; Shen and Fan, 1950. As "G. clavatum": Okamura, 1934a, 1935a, 1935b, 1936.

Gelidium latiusculum Okamura, 1935b:443. Chiang, 1962a; Fan, 1951, 1953a; Okamura, 1935b, 1936; Shen and Fan, 1950.

Gelidium pacificum Okamura, 1914:99.

Okamura, 1915b; Oshima, 1915.

Gelidium planiusculum Okamura, 1935b:442.

Chiang, 1962a; Fan, 1951, 1953a; Okamura, 1935b, 1936; Santelices and Stewart, 1985; Shen and Fan, 1950.

Gelidium pusillum (Stackhouse) Le Jolis, 1863:139.

Chiang, 1962a; Fan, 1951; Santelices and Stewart, 1985; Taniguti, 1976. As "Acrocarpus pusillus": Martens, 1866.

Gelidium pusillum f. foliaceum Okamura, 1934a:51.

Ariga, 1920; Taniguti, 1976.

Gelidium subcostatum Okamura in Schmitz, 1894:1.

Ariga, 1919; Horikawa, 1919.

Gelidium yamadae (Okamura) Fan, 1951:10. Fan, 1951, 1953a. As "G. densum": Okamura, 1935b; Shen and Fan, 1950.
Remarks: Fan (1951) considers $G$. densum invalid on the basis of an earlier homonym.

Gelidium sp.

Ariga, 1920.

Pterocladia nana Okamura, 1934a:64.

Chiang, 1962a, 1973b; Fan, 1951.

Pterocladia tenuis Okamura, 1934a:62.

Arasaki, 1964; Chiang, 1962a, 1962b, 1973b; Fan, 195 l, 1953a; Okamura, 1935b, 1936; Segawa, 1974; Rho, 1958; Shen and Fan, 1950; Taniguti, 1976. As "Gelidium corneum var. pinnatum": Heydrich, 1894. As "P. capillaceum": Ariga, 1920; Chihara, 1970, 1975; Okamura, $1915 \mathrm{~b}$; Oshima, 1915.

\section{Gelidiellaceae}

Gelidiella acerosa (Forsskål) Feldmann et Hamel, 1934:533. A riga, 1920; Chiang, 1973b; Chihara, 1975; Okamura, 1936; Segawa, 1974; Shen and Fan, 1950; Yoshikawa and Yoshikawa, 1977. As "Gelidiopsis rigida": Okamura, 1931 .

\section{CRYPTONEMIALES}

\section{DUMONTIACEAE}

Dudresnaya japonica Okamura, 1908:209.

Shen and Fan, 1950.

\section{RHIZOPHYLLIDACEAE}

Remarks: Placed in the Cryptonemiales By Kylin (1956), this family was later transferred by Wiseman (1975) to the Gigartinales (see also Kraft (1981) and Wynne and Kraft (1981)). However, West and Hommersand (1981) noted "there is no major evidence to suggest that the [Rhizophyllidaceae] is closely related to either order," and retained it in the Cryptonemiales.

Chondrococcus hornemannii (Mertens) Schmitz, 1895:170, 'hornemanni'.

Arasaki, 1964; Okamura, 1931, 1936; Segawa, 1974; Shen and Fan, 1950. As "Desmia hornemannii": Chiang, 1962a, 1973b; Chihara, 1970; Su et al., 1982; Yoshikawa and Yoshikawa, 1977.

Rhodopeltis borealis Yamada, 1931a:75.

Arasaki, 1964; Chihara, 1975; Nozawa, 1963, 1970; Okamura, 1936; Segawa, 1974; Shen and Fan, 1950; Yamada, 1931 a.

Rhodopeltis gracilis Yamada et Tanaka in Yamada, 1935:30. Arasaki, 1964; Nozawa, 1963, 1970; Okamura, 1936; Shen and Fan, 1950; Yamada, 1935.

Rhodopeltis setchelliae Yamada, 1935:33, 'setchellii'.

Nozawa, 1963, 1970; Okamura, 1936; Shen and Fan, 1950; Yamada, 1935. 


\section{Peyssonneliaceae}

Peyssonnelia caulifera Okamura, 1899:8.

Ariga, 1920.

Peyssonnelia distenta (Harvey) Yamada, 1930:29.

Ariga, 1920; Chiang, 1962a, 1962b; Okamura, 1899, 1936; Shen and Fan, 1950; Yamada, 1930; Yoshikawa and Yoshikawa, 1977. As "P. involvens": Okamura, 1909.

Peyssonnelia rubra (Greville) J. Agardh, 1852:502.

De Toni, 1895; Heydrich, 1894; Okamura, 1897, 1931, 1936; Shen and Fan, 1950.

\section{Corallinaceae}

Amphiroa beauvoisii Lamouroux, 1816:299.

As "A. pusilla": Okamura, 1936; Segawa, 1974; Shen and Fan, 1950. As "A. zonata": Chiang, 1962a.

Remarks: Recently Norris and Johansen (1981:6) considered $A$. zonata to be a taxonomic synonym of $A$. beauvoisii Lamouroux.

Amphiroa bowerbankii Harvey, 1849b:97.

De Toni, 1895; Heydrich, 1894; Shen and Fan, 1950.

Amphiroa ephedracea (Lamarck) Decaisne, 1842:124.

Chihara, 1970; De Toni, 1895; Okamura, 1936; Rho, 1958; Segawa, 1974; Shen and Fan, 1950. As "A. exilis": Heydrich, 1894; Yendo, 1902.

Amphiroa fragilissima (Linnaeus) Lamouroux, 1816:298.

Yoshikawa and Yoshikawa, 1977.

Amphiroa multifida Kützing, 1858:27.

De Toni, 1895; Heydrich, 1894; Shen and Fan, 1950.

Amphiroa pusilla Yendo, 1902:13.

Rho, 1958; Su et al., 1982.

Amphiroa spp.

Ariga, 1920.

Cheilosporum anceps Yendo, 1902:18.

Shen and Fan, 1950.

Cheilosporum jungermannioides Ruprecht in J. Agardh, 1852:546.

Chihara, 1975; Okamura, 1931, 1936; Segawa, 1941, 1974; Shen and Fan, 1950; Tseng, 1983. As "Amphiroa cultrata var. globulifera": De Toni, 1895; Heydrich, 1894. As "C. cultratum": Yendo, 1902.

Jania adhaerens Lamouroux, 1816:270.

Chiang, 1962a, 1962b; Okamura, 1931, 1936; Shen and Fan, 1950; Su et al., 1982; Taniguti, 1976. As "Corallina adhaerens": De Toni, 1905; Heydrich, 1894; Yendo, 1902. As "Corallina decussato-dichotoma": Okamura, 1931, 1936; Segawa, 1974. As "J. decussato-dichotoma": Arasaki, 1964; Chiang, 1962a; Chihara, 1970, 1975; Shen and Fan, 1950; Taniguti, 1976.

Jania radiata Yendo, 1902:26.

Chiang, 1962a.

Jania tenella Kützing, 1858:41.

Chiang, 1962a.
Jania undulata Yendo, 1902:26.

Shen and Fan, 1950.

Lithophyllum perulatum Foslie, 1900:18.

De Toni, 1895; Okamura, 1936; Shen and Fan, 1950;

Yendo, 1902. As "Melobesia pustulata": Heydrich, 1894.

Lithophyllum spp.

Ariga, 1920.

Lithothamnium membranaceum (Esper) Foslie, 1905:72.

De Toni, 1895; Okamura, 1936; Shen and Fan, 1950.

As "Melobesia membranaceae": Heydrich, 1894; Yendo, 1902.

Lithothamnium spp.

Ariga, 1920.

Mastophora pygmaea Heydrich, 1894:300.

De Toni, 1895; Fan, 1974; Heydrich, 1894; Okamura, 1936; Shen and Fan, 1950; Yendo, 1902.

Remarks: Fan $(1974: 251,254)$ considers the taxonomy of this species suspect and encourages study of the type specimen and type-locality specimens to confirm the identity of Heydrich's plant.

Mastophora rosea (C. Agardh) Setchell, 1943:129.

Chiang, 1973b; Chihara, 1975; Segawa, 1974; Su et al., 1982; Taniguti, 1976; Yoshikawa and Yoshikawa, 1977.

As "M. macrocarpa": Chiang, 1962a; De Toni, 1905;

Heydrich, 1894; Okamura, 1931, 1936; Shen and Fan, 1950; Yendo, 1902.

Melobesia farinosa Lamouroux, 1816:315.

De Toni, 1895; Heydrich, 1894; Okamura, 1936; Shen and Fan, 1950; Yendo, 1902.

Melobesia spp.

Ariga, 1920.

Tenarea tumidulum (Foslie) Adey, 1970:7.

As "Dermatolithon tumidulum": Chiang, 1973a; Okamura, $1935 \mathrm{~b}$.

\section{ENDOCLADiaCEAE}

Gloiopeltis complanata (Harvey) Yamada, 1932a: 117. As "Endocladia complanata": Ariga, 1920. As "Gloiopeltis cervicornis:" Ariga, 1921.

Gloiopeltis furcata (Postels et Ruprecht) J. Agardh, 1851:235.

Chiang, 1973b; Fan, 1953a; Shen and Fan, 1950.

Gloiopeltis tenax (Turner) J. Agardh, 1842:68.

Chiang, 1969, 1973a; Okamura, 1935b.

\section{Cryptonemiaceae}

Carpopeltis angusta (Harvey) Okamura, 1910:66. Ariga, 1920; Shen and Fan, 1950.

Carpopeltis cornea Okamura, 1936:553. Arasaki, 1964; Chihara, 1975; Okamura, 1936; Rho, 1958; Segawa, 1974; Shen and Fan, 1950; Su et al., 1982. 
Carpopeltis flabellata (Holmes) Okamura, 1935a:39.

Ariga, 1920; Chiang 1962a.

Carpopeltis formosana Okamura, 1931:110.

Fan, 1953a; Okamura 1931, 1936; Shen and Fan, 1950;

Tokida, 1939.

Carpopeltis rigida (Harvey) Schmitz, 1895:168.

Ariga, 1920; Chihara, 1975; Okamura, 1909, 1930, 1936; Rho, 1958; Segawa, 1974; Shen and Fan 1950; Yoshikawa and Yoshikawa, 1977.

Grateloupia carnosa Yamada et Segawa in Yamada, 1938b: 126.

Chiang, 1962a.

Grateloupia filicina (Wulfen) J. Agardh, 1851:180.

Ariga, 1919, 1920; Chiang, 1962a, 1973b; De Toni, 1895 ; Horikawa, 1919.

Grateloupia filicina f. filiformis (Kützing) Pilger, 1911:310.

As "G. filiformis": Martens, 1866.

Grateloupia okamurae Yamada, 1941:204, 'okamurai'.

Chiang, 1973a.

Grateloupia ramosissima Okamura, 1913:60.

Chiang, 1962a, 1973a; Chihara, 1970, 1975; Dawson, 1954c; Rho, 1958; Segawa, 1974; Shen and Fan, 1950.

Halymenia ceylanica (Harvey) Kützing, 1866:33.

As "H. formosa": Okamura, 1909. As "H. durvillaei var. formosa": Tokida, 1939. As "H. durvillaei var. ceylanica": Ariga, 1920; Chiang, 1962a; Okamura, 1936; Shen and Fan, 1950.

Halymenia durvillaei Bory, 1826, pl. 15. Fan, 1953a.

Polyopes polyideoides Okamura, 1895:447.

Chiang, 1973a, 1973b; Okamura, 1935 b.

Polyopes sp.

Heydrich, 1894.

\section{GIGARTINALES}

\section{SOLIERIACEAE}

Eucheuma arnoldii Weber-van Bosse, 1928:421. Kraft, 1972. As “E. cupressoideum": Shen and Fan, 1950.

Eucheuma "audiolis". Tseng and Chen, 1977; Tseng, 1984.

Remarks: This species name is apparently a nomen nudum (fide M.S. Doty).

Eucheuma cottonii Weber-van Bosse, 1913b:115. Tseng and Chen, 1977.

Eucheuma crassum Zanardini, 1878:36. Shen and Fan, 1950; Yamada, 1936a.

Eucheuma crustaeforme Weber-van Bosse, 1928:415. Okamura, 1931, 1936. As “E. cottonii”: Okamura, 1931.

Eucheuma gelatinae (Esper) J. Agardh, 1852:628. Chihara, 1975; Doty and Norris, 1985; Shen and Fan, 1950; Tseng, 1983; Tseng and Chen, 1977; Yamada, $1936 a$.
Eucheuma muricatum (Gmelin) Weber-van Bosse, 1928:413. Higashi, 1934; Shen and Fan, 1950.

Eucheuma muricatum f. depauperata Weber-van Bosse, 1928:415.

Ariga, 1920; Okamura, 1936. As "E. muricatum": Okamura, 1931. As "E. spinosum": Okamura, 1909, 1915b.

Eucheuma okamurae Yamada, 1936a:125, 'okamurai'.

Shen and Fan, 1950.

Eucheuma papulosa Cotton et Yendo in Cotton, 1914:220. Cotton, 1915 ; Tilden, 1929.

Eucheuma serra J. Agardh, 1852:626.

Chiang, 1962a, 1973b; Chihara, 1975; Segawa, 1974; Shen and Fan, 1950; Su et al., 1982; Tseng and Chen, 1977; Yamada, 1936a; Yoshikawa and Yoshikawa, 1977.

Eucheuma spp.

Ariga, 1920.

Meristotheca coacta Okamura, 1930:97.

Arasaki, 1964; Okamura, 1930, 1936; Segawa, 1974; Yoshikawa and Yoshikawa, 1977.

Meristotheca papulosa (Montagne) J. Agardh, 1876:584.

Arasaki, 1964; Chiang, 1962a; Fan, 1953a; Okamura, 1936; Segawa, 1974; Tseng, 1983.

\section{HyPNEACEAE}

Hypnea boergesenii Tanaka, 1941:233.

Chiang, 1962a; Fan, 1953a; Shen and Fan, 1950; Tanaka, 1941; Tseng, 1983.

Hypnea cenomyce J. Agardh, 1852:452.

Shen and Fan, 1950; Tanaka, 1941; Yoshikawa and Yoshikawa, 1977.

Hypnea cervicornis J. Agardh, 1852:451.

Yoshikawa and Yoshikawa, 1977.

Hypnea charoides Lamouroux, 1813:131.

Chiang, 1973b; Fan, 1953a; Shen and Fan, 1950; Tseng, 1983.

Hypnea chordacea Kützing, 1847:776.

Arasaki, 1964; Shen and Fan, 1950; Tanaka, 1941.

Hypnea chordacea f. simpliciuscula (Okamura) Tanaka, 1941:232.

Chiang, 1962a; Chihara, 1975; Shen and Fan 1950; Tanaka, 1941.

Hypnea cornuta (Lamouroux) J. Agardh, 1852:449.

Shen and Fan, 1950; Tanaka, 1941.

Hypnea esperi Bory, 1828:157.

Shen and Fan, 1950; Tanaka, 1941.

Hypnea hamulosa (Turner) Lamouroux, 1813:44.

Okamura, 1931, 1936; Shen and Fan, 1950; Tanaka, 1941 .

Remarks: Several authors (e.g., J. Agardh, 1852:447; De Toni 1924:477; Okamura, 1936:611; Tanaka, 1941:245; Shen and Fan, 1950:339; Weber-van Bosse, 1928:453; and Zanardini, 1858:270) have incorrectly 
cited "Montagne 1850:n.16 (page 9)" as the original place of publication.

Hypnea japonica Tanaka, 1941:236.

Chiang, 1962a; Chihara, 1970; Segawa, 1974; Shen and Fan 1950; Tanaka, 1941; Tseng, 1983.

Hypnea nidulans Setchell, 1924:161.

Chihara, 1975; Okamura, 1931, 1936; Shen and Fan, 1950; Su et al., 1982; Tanaka, 1941; Yoshikawa and Yoshikawa, 1977.

Hypnea pannosa J. Agardh, 1847:14.

Okamura, 1931, 1936; Shen and Fan, 1950; Tanaka, 1941; Yoshikawa and Yoshikawa, 1977.

Hypnea saidana Holmes, 1896:256.

Chiang, 1973b.

Hypnea seticulosa J. Agardh, 1852:446.

Ariga, 1920; Okamura, 1931, 1936; Tokida, 1939.

Hypnea spinella (Greville) Kützing, 1849:759.

Chiang, 1962a.

Hypnea sp.

Su et al., 1982.

\section{Plocamiaceae}

Plocamium oviforme Okamura, 1896:23.

Ariga, 1920.

Plocamium serratulum Okamura, 1932:100.

Okamura, 1936; Shen and Fan, 1950. As "P. costatum":

Okamura, 1923, 1931; Yendo, 1918.

Plocamium telfairiae (J. Agardh) Harvey in Kützing, 1849:885.

Shen and Fan, 1950; Yendo, 1915. As "P. abnorme": Ariga, 1919, 1920; Horikawa, 1919; Okamura, 1931.

Plocamium telfairiae f. uncinatum Okamura, 1936:615.

As "P. abnorme f. uncinatum": Okamura, 1913.

\section{GraCilariaCEAE}

Ceratodictyon spongiosum Zanardini, 1878:37.

Arasaki, 1964; Ariga, 1920; Okamura, 1936. As " $C$. spongioides": Okamura, 1931; Shen and Fan, 1950.

Gelidiopsis hachijoensis Yamada et Segawa, 1953:112. Yoshikawa and Yoshikawa, 1977.

Gelidiopsis repens (Kützing) Schmitz, 1895:148.

Chiang, 1962a, 1973b; Chihara, 1975; Okamura, 1931, 1936; Shen and Fan, 1950; Taniguti, 1976; Yamada 1932b; Yoshikwa and Yoshikawa, 1977.

Gelidiopsis variabilis (Greville) Schmitz, 1895:148.

Yamada, 1932b.

Gracilaria arcuata Zanardini, 1858:265.

Chang and Xia, 1976; Chiang, 1973a, 1973b, 1985;

Okamura, 1935b; Su et al., 1982.

Gracilaria blodgettii Harvey, 1853:111.

Chiang, 1985; Ohmi, 1958; Yang and Chiang, 1982.

Remarks: Although "G. blodgettii" has been reported from Taiwan (Chiang, 1985), the South China Sea (Xia, 1985), and southern Japan (Yamamoto, 1985), the relationship between them and Caribbean type specimens of G. blodgettii Harvey is still to be resolved.

Gracilaria bursapastoris (Gmelin) Silva, 1952:265.

As "G. compressa": Chen, 1976; Shang, 1976.

Gracilaria canaliculata (Kützing) Sonder, 1871:56.

As "Corallopsis opuntia": Arasaki, 1964; Segawa, 1974.

Gracilaria chorda Holmes, 1896:253.

Ariga, 1920; Chen, 1976.

Gracilaria coronopifolia J. Agardh, 1852:592.

Chang and Xia, 1976; Chiang, 1985; Ohmi, 1958;

Tseng, 1983; Yamada, 1941; Yang and Chiang, 1982.

As "G. lichenoides f. coronopifolia": Fan, 1953a.

Gracilaria crassa Harvey ex J. Agardh, 1876:417.

Chang and Xia, 1976; Chihara, 1975; Chiang, 1985;

Ohmi, 1958; Okamura, 1936; Shen and Fan, 1950;

Tseng, 1983; Yamada, 1933; Yang and Chiang, 1982.

Gracilaria denticulata (Kützing) Schmitz in Mazza, 1907:138.

Chiang, 1962a, 1985; Chihara, 1975; Okamura, 1931, 1936 ; Yang and Chiang, 1982.

Gracilaria edulis (Gmelin) Silva, 1952:293.

Nelson et al., 1983. As "G. lichenoides": Chen, 1976; Chiang, 1981; Shang, 1976.

Gracilaria eucheumioides Harvey, 1859:331.

Chang and Xia, 1976; Chiang, 1973a, 1985; Okamura, 1935b; Yang and Chiang, 1982.

Gracilaria gigas Harvey, 1859:330.

Chiang, 1981; Chen, 1976; Shang, 1976.

Gracilaria incurvata Okamura, 1931:41.

Chiang, 1973a; Okamura, 1935b.

Gracilaria punctata (Okamura) Yamada, 1941:203.

Ohmi, 1958; Shen and Fan, 1950; Yamada, 1941.

Gracilaria purpurascens Harvey in J. Agardh, 1885:63.

Ohmi, 1958; Shen and Fan, 1950; Yamada, 1938b; Yoshikawa and Yoshikawa, 1977.

Gracilaria spinulosa (Okamura) Chang et Xia, 1976:148.

Tseng, 1983. As "G. purpurascens f. spinulosa": Ohmi, 1958; Shen and Fan, 1950; Yamada, 1941; Yoshikwa and Yoshikwa, 1977.

Gracilaria salicornia (C. Agardh) Dawson, 1954b:4. Chiang, 1985; Yang and Chiang, 1982.

Gracilaria "verrucosa".

G. verrucosa sensu Chiang, 1981, 1985; Nelson et al., 1983; Rho, 1958; Segawa, 1974; Yang et al., 1981; Yang and Chiang, 1982 [non G. verrucosa (Hudson) Papenfuss, 1950:195]. As "G. confervoides": De Toni, 1895; Fan, 1953a; Martens, 1866; Shang, 1976; Shen and Fan, 1950; Tokida, 1954.

Remarks: A comparison with type-locality (England) specimens of $G$. verrucosa showed the Taiwan specimens are a different species (Chiang, 1985; Abbott et al., 1985), and that the Chinese and Japanese specimens identified 
as " $G$. verrucosa" are the same (Xia and Yamamoto, 1985; Yamamoto, 1985; Xia, 1985) and that they too are different from the British $G$. verrucosa. Recently, Zhang and Xia (1985:177) described $G$. asiatica for the specimens from Japan and China.

Gracilaria sp.

Ariga, 1920.

\section{SPHAERococcaceae}

Caulacanthus okamurae Yamada, 1933:278, 'okamurai'.

Rho, 1958; Taniguti, 1976; Yamada, 1933.

Caulacanthus spinellus (Hooker et Harvey) Kützing, 1849:753.

Chiang, 1973b; Shen and Fan, 1950; Yamada, 1933.

Caulacanthus ustulatus var. fastigiatus (Kützing) Pilger, 1920:5.

As “C. fastigiatus": Martens, 1866.

Phacelocarpus japonicus Okamura, 1902:79.

Shen and Fan, 1950.

\section{Sarcodiaceae}

Sarcodia ceylanica Harvey ex Kützing, 1869, pl. 33.

Chiang, 1962a; Shen and Fan, 1950; Yoshikawa and Yoshikawa, 1977.

\section{Phyllophoraceae}

Ahnfeltia paradoxa (Suringar) Okamura, 1934b:13. As "Gymnogongrus paradoxus": Ariga, 1920.

Gymnogongrus flabelliformis Harvey, $1856: 332$.

Shen and Fan, 1950; Taniguti, 1976.

\section{Gigartinaceae}

Chondrus crispus Stackhouse, 1797:xxiv.

Ariga, 1920; Mikami, 1965.

Chondrus ocellatus Holmes, 1896:252.

Chiang, 1973b; Fan, 1953a; Tseng, 1983. As "C. ocellatus

f. typicus": Chiang, 1962a.

Chondrus ocellatus f. canaliculatus Okamura, 1932:84.

Shen and Fan, 1950.

Gigartina intermedia Suringar, 1870:30.

Ariga, 1921; Chiang, 1962a; Fan, 1953a; Shen and Fan, 1950; Taniguti, 1976.

Gigartina ochotensis Ruprecht in Kjellman, 1889:31.

Ariga, 1920.

Gigartina tenella Harvey, 1859:331.

Arasaki, 1964; Okamura, 1930, 1936; Rho, 1958; Shen and Fan, 1950.

Rhodoglossum affine (Harvey) Kylin, 1928:49.

As “Chondrus affinis": Heydrich, 1894.

\section{RHODYMENIALES}

\section{RHODYMENIACEAE}

Chrysymenia procumbens Weber-van Bosse, 1928:470.

Okamura, 1931, 1936; Shen and Fan, 1950.

Erythrocolon podagrica (Harvey ex J. Agardh in Grunow) J. Agardh ex Kylin, 1931:14.

Shen and Fan, 1950.

Remarks: For discussion on the complicated nomenclature of this taxon see Abbott and Littler (1969:168).

Rhodymenia spinulosa Okamura, $1934 \mathrm{~b}: 33$.

Okamura, 1934b, 1936; Yoshikawa and Yoshikawa, 1977.

Weberella micans Hauptfleisch in Schmitz and Hauptfleisch, 1897:402.

Arasaki, 1964; Okamura, 1936; Segawa, 1974; Shen and Fan, 1950; Yamada, 1932 b.

\section{Champiaceae}

Champia parvula (C. Agardh) Harvey, 1853:76.

Chiang, 1962a; Higashi, 1934; Okamura, 1931, 1936; Shen and Fan, 1950.

\section{CERAMIALES}

\section{Ceramiaceae}

Carpoblepharis schmitziana var. erecta Yamada, 1932b:273.

Okamura, 1936; Shen and Fan, 1950.

Carpoblepharis warburgii Heydrich, 1894:297.

De Toni, 1895; Heydrich, 1894; Okamura, 1936; Shen and Fan, 1950.

Remarks: Hommersand (1963:196) and Fan (1974) noted " $C$. warburgii has never been re-examined, but . . . it appears unlikely that this taxon belongs in Carpoblepharis."

Centroceras clavulatum (C. Agardh) Montagne, 1846:140.

Ariga, 1920; Chiang, 1962a, 1962b, 1973b; Chihara, 1970, 1975; De Toni, 1895; Okamura, 1931, 1936; Rho, 1958; Segawa, 1974; Shen and Fan, 1950; Taniguti, 1976; Yamada, 1928; Yoshikawa and Yoshikawa, 1977.

As "Ceramium clavulatum": Heydrich, 1894.

Centroceras minutum Yamada, 1944c:42.

Chiang, 1962a.

Ceramium aduncum Nakamura, 1950:159.

Nakamura, 1950, 1965; Itono, 1972, 1977b. As "Ceramium clarionense": Dawson, 1954c.

Ceramium ciliatum (Ellis) Ducluzeau, 1805:64, var. robustum (J. Agardh) Mazoyer, 1938:322.

Nakamura, 1965; Itono, 1972, 1977b.

Ceramium flaccidium (Kützing) Ardissone, 1971:40.

As "C. gracillimum var. byssoideum": 1tono, 1972, $1977 \mathrm{~b}$. 
Remarks: See Womersley (1978:234-238) for discussion on the nomenclature and toxonomy of this taxon.

Ceremium gracillimum (Kützing) Griffiths et Harvey in Harvey, $1848 \mathrm{~b}$, pl. 206.

Nakamura, 1965; Yoshikawa and Yoshikawa, 1977. Remarks: Womersley (1979:234) considers C. gracillimum to be a synonym of C. flaccidium (Kützing) Ardissone.

Ceramium nakamurai Dawson, 1954a:6.

Nakamura, 1965; 1tono, 1972, 1977b. As “C. equisetoides": Nakamura, 1950.

Ceramium paniculatum Okamura, 1896:36.

Nakamura, 1965.

Ceramium tenerrimum (Martius) Okamura, 1921:112.

Chiang, 1962a; Okamura, 1936; Rho, 1958; Shen and Fan, 1950; 1tono, 1972, 1977b.

Ceramium tenuissimum (Lyngbye) J. Agardh, 1851:120.

Ariga, 1921 ; De 'Toni, 1895; Heydrich, 1894; Okamura, 1936; Rho, 1958; Shen and Fan, 1950; Tseng, 1983; Yamada, 1928.

Dasyphila plumarioides Yendo, 1920:7.

Arasaki, 1964; 1tono, 1977b; Okamura, 1923, 1931, 1936; Segawa, 1974; Yendo, 1920.

Gymnothamnion elegans (C. Agardh) J. Agardh, 1892:27.

ltono, 1977b. As "Plumaria ramosa": Okamura, 1936;

Shen and Fan, 1950; Yamada and Tanaka, 1934.

Microcladia elegans Okamura 1907:1.

Chiang, 1962a.

Ptilothamnion cladophorae (Yàmada et Tanaka) FeldmannMazoyer, 1941:375.

Fan, 1974; 1tono, 1977b. As "Spermothamnion cladophorae": Okamura 1936, 1937; Shen and Fan, 1950;

Yamada and Tanaka, 1934.

Reinboldiella schmitziana (Reinbold) De Toni, 1895:35.

Ariga, 1920.

Spyridia filamentosa (Wulfen) Harvey ex Hooker, 1833:337.

Ariga, 1920; Chihara, 1975; De Toni, 1895; Heydrich,

1894; Okamura, 1913, 1936; Rho, 1958; Segawa, 1974;

Shen and Fan, 1950.

Tiffaniella codicola (Yamada et Tanaka) Doty et Meñez, $1960: 137$.

Fan, 1974; ltono, 1977b. As "Spermothamnion codicola": Okamura, 1936, 1937; Shen and Fan, 1950; Yamada and Tanaka, 1934.

Wrangelia velutina Harvey, $1854: 546$.

Okamura, 1931; Shen and Fan, 1950.

\section{Delesseriaceae}

Caloglossa bombayensis B $\phi$ rgesen, 1933:127.

Fan, 1952.

Claudea batanensis Tanaka, 1967:18.

Tanaka, 1967; Yoshikawa and Yoshikawa, 1977.

Haloplegma duperreyi Montagne, 1842:258.

Yamada, 1936b; Tseng, 1983.
Holmesia neurymenioides Okamura, 1932:98.

Okamura, 1936; Shen and Fan, 1950.

Martensia denticulata Harvey, 1854:537.

Ariga, 1919, 1920; Horikawa, 1919.

Martensia flabelliformis Harvey ex J. Agardh, 1863:826.

Chiang, 1962b; Chihara, 1975; Segawa, 1974; Shen and Fan, 1950; Yamada, 1936b.

Nitophyllum sp.

Ariga, 1920.

\section{DasyaCeae}

Dasya sp.

Ariga, 1919, 1920; Horikawa, 1919.

\section{RHODOMELACEAE}

Acanthophora aokii Okamura, 1934b:35.

Okamura, 1934b, 1936; Shen and Fan, 1950.

Acanthophora muscoides (Linnaeus) Bory, 1828:156.

Shen and Fan, 1950.

Acanthophora orientalis (Sonder) J. Agardh, 1863:820.

Ariga, 1920; Chiang, 1962a, 1973b; Chihara, 1975; Fan, 1953a; Okamura, 1931, 1936; Segawa, 1974; Shen and Fan, 1950; Tokida, 1939; Yoshikawa and Yoshikawa, 1977.

Acrocystis nana Zanardini, 1872:145.

Chiang, 1973b; Chihara, 1975; Okamura, 1931, 1936; Segawa, 1974; Shen and Fan, 1950; Tseng, 1983.

Amansia glomerata C. Agardh, 1822:194.

Ariga, 1920; Chiang, 1973b; Chihara, 1975; Okamura, 1930, 1931, 1936; Segawa, 1974; Shen and Fan, 1950; Yoshikawa and Yoshikawa, 1977.

Bostrychia tenella (Vahl) J. Agardh, 1863:869.

Ariga, 1920; Chiang, 1962a; Taniguti, 1976; Yoshikawa and Yoshikawa, 1977.

Chondria armata (Kützing) Okamura, 1907:69.

Ariga, 1919, 1920; Chiang, 1962a, 1973b; Chihara, 1970, 1975; Fan, 1953a; Horikawa, 1919; Okamura, 1930, 1931, 1936; Segawa, 1974; Shen and Fan, 1950; Tokida, 1939; Tseng, 1983.

Chondria dasyphylla (Woodward) C. Agardh, 1822:350.

Ariga, 1920; De Toni, 1895; Yoshikawa and Yoshikawa, 1977. As "Laurencia dasyphylla": Martens, 1866.

Digenia simplex (Wulfen) C. Agardh, 1822:389.

Ariga, 1920; Chihara, 1970, 1975; Fan, 1953a; Higashi, 1934; Okamura, 1931, 1936; Rho, 1958; Segawa, 1974; Shen and Fan, 1950; Tseng, 1983.

Herposiphonia subdisticha Okanura, 1899:37.

Chiang, 1962a; Okamura, 1931, 1936; Shen and Fan, 1950.

Herposiphonia sp.

Chiang, 1962b.

Laurencia brongniartii J. Agardh, 1841:20.

Remarks: Specimens from Nanwan, Taiwan (collected 
by Ger Dzeng-Joung; 21 August 1979; \#US-071851), were identified by J. Norris as L. brongniartii and establish the presence of this taxon in southern Taiwan.

Laurencia flexilis var. tropica (Yamada) Xia et Zhang, 1982:538.

Tseng, 1983.

Laurencia forsteri (Mertens ex Turner) Greville, 1830:lii. Ariga, 1920.

Laurencia glandulifera Kützing, 1849:855.

Ariga, 1920.

Laurencia grevilleana Harvey, 1854:545.

Ariga, 1920, Su et al., 1982.

Remarks: Saito and Womersley (1974:839) considered $L$. grevilleana to be a taxonomic synonym of $L$. brongniartii J. Agardh.

Laurencia obtusa var. densa Yamada, 1931 b:226.

Dawson, 1954c; Shen and Fan, 1950; Yamada, 1931b, 1936c.

Laurencia palisada Yamada, 193 lb:196.

Chiang, 1962a; Shen and Fan, 1950; Su et al., 1982; Yamada, 1931b, 1936c.

Laurencia papillosa (Forsskål) Greville, 1830:lii. Ariga, 1920; Chihara, 1975; Cotton, 1915; Okamura, 1931; Shen and Fan, 1950; Tilden, 1929; Yamada, 1936c; Yoshikawa and Yoshikawa, 1977.

Laurencia perforata (Montagne) J. Agardh, 1876:648. Ariga, 1920; De Toni, 1895; Heydrich, 1894.

Laurencia pinnatifida (Gmelin) Lamouroux, $1813: 42$. As "L. pinnatifida var. simplex": De Toni, 1895; Heydrich, 1894.

Laurencia tropica Yamada, 193 lb:223.

Okamura, 1931; Shen and Fan, 1950; Taniguti, 1976; Yamada, 193 lb, 1936c.

Laurencia venusta Yamada, 1931 b:203.

Okamura, 1931; Shen and Fan, 1950; Yamada, 1936c.

Laurencia sp.

Ariga, 1919; Fan, 1953a; Horikawa, 1919; Taniguti, 1976; Tokida, 1939.

Leveillea jungermannioides (Martius et Hering) Harvey, 1854:539.
Ariga, 1920; De Toni, 1895; Chiang, 1962b; Heydrich, 1894; Okamura, 1931, 1936; Rho, 1958; Shen and Fan, 1950; Tseng, 1983; Yoshikawa and Yoshikawa, 1977. As

"Polyzonia jungermannioides": Okamura, 1897.

Murrayella periclados (C. Agardh) Schmitz, 1893:227.

Tokida, 1941.

Murrayella squarrosa (Harvey) Schmitz, 1893:228.

Shen and Fan, 1950; Yamada, 1936b.

Neurymenia fraxinifolia (Mertens ex Turner) J. Agardh, 1863:1135.

Chiang, 1973b; Okamura, 1931; Shen and Fan, 1950;

Tanaka and ltono, 1969.

Polysiphonia harlandii Harvey, 1859:330.

Segi, 1951; Tseng, 1944, 1983; Yamada, 1933; Yoshikawa and Yoshikawa, 1977.

Polysiphonia kampsaxii B $\phi$ rgesen, 1939:122.

Segi, 1951.

Polysiphonia pulvinata J. Agardh, 1842:124.

Segi, 1951.

Symphyocladia marchantioides (Harvey) Falkenberg, 1901: 277.

Ariga, 1920; Chiang 1962a; Chihara, 1970, 1975; Okamura 1930, 1931, 1936; Rho, 1958; Segawa, 1974; Shen and Fan, 1950; Tseng, 1983. As "Placophora marchantioides": Heydrich, 1894.

Vidalia obtusiloba (Mertens) J. Agardh, 1863:1123.

Ariga, 1920; Chihara, 1975; Okamura, 1931, 1936; Shen and Fan, 1950.

\section{INCERTAE SEDIS}

\section{WURDEMANNIACEAE}

Remarks: Taylor (1960:348, 361, 633) assigned the Wurdemanniaceae to the Gelidiales; however, Farr et al. (1979) have referred Wurdemannia to incertae sedis.

Wurdemannia setacea Harvey, 1853:246.

Okamura, 1931, 1936; Shen and Fan, 1950.

Wurdemannia sp.

Okamura, 1931. 


\section{Literature Cited}

Abbott, I.A

1970. Yamadaella, a New Genus in the Nemaliales (Rhodophyta). Phycologia, 9:115-123.

1976. Dotyophycus pacificum gen. et sp. nov., with a Discussion of Some Families of Nemaliales (Rhodophyta). Phycologia, I 5:1 25-1 32.

1984. Two New Species of Liagora (Nemaliales, Rhodophyta) and Notes on Liagora farinosa Lamouroux. American Journal of Botany, 7 I: 1015-1022.

Abbott, I.A., and G.J. Hollenberg

1976. Marine Algae of California. xii +827 pages. Stanford, California: Stanford University Press.

Abbott, 1.A., and M.M. Littler

1969. Some Rhodymeniales from Hawaii. Phycologia, 8:165-169.

Abbott, 1.A., and M. Yoshizaki

1981. A Second Species of Dotyophycus (Nemaliales, Rhodophyta) That Emphasizes the Distincmess of a Diffuse Gonimoblast. Phycologia, 20:222-227.

Abbott, 1.A., Y.M. Chiang, S. Fredericq, J.N. Norris, R.T. Tsuda, B.M. $X i a$, and $H$. Yamamoto

I985. The Red Alga Gracilaria Greville (Gracilariaceae, Gigartinales): Introduction. In 1.A. Abbott and J.N. Norris, editors, Taxonomy of Economic Seaweeds ..., pages 67-68. La Jolla, California: California Sea Grant College Program, University of California, La Jolla.

Adey, W.H.

1970. A Revision of the Foslie Crustose Coralline Herbarium. Det Kongelige Norske Videnskabers Selskab Skrifter, I:1-46.

Agardh, C.A.

1820. Species algarum Volume 1, part 1, . . 168 pages. Lund: Berling.

1822. Species algarum .... Volume 1, part 2, [v-vi] + I69-398 pages. Lund: Berling.

1823. Species algarum ... Volume 1, part 2, [vii-viii] + 399-531 pages. Greifswald: Ernst Mauritius.

1824. Systema algarum. xxxviii $+3 I 2$ pages. Lund: Berling.

Agardl,, J.G

1841. In historiam algarum symbolae. Linnaea, 15:1-50, 443-457.

1842. Algae maris Mediterranei et Adriatici ... x +164 pages. Paris: Fortin, Masson.

1847. Nya alger från Mexico. Öfuersight af Kongliga Vetenskaps-Akademiens Förhandlingar, 4(1):5-17.

1848. Species genera et ordines algarum, seu descriptiones succinctae specierum, generum et ordinum, quibus algarum regnum constituitur. Volume 1, viii +363 pages. Lund: C.W.K. Gleerup.

1851. Species genera et ordines algarum .... Volume 2, part I, xii +336 $+[14]$ pages. Lund: C.W.K. Gleerup.

1852. Species genera et ordines algarum ... Volume 2, part 2, [2] + $337-700+[20]+701-786$ pages. Lund: C.W.K. Gleerup.

1863. Species genera et ordines algarum .... Volume 2, part 3, fascicle 2. [2] + 787-1291 pages. Lund: C.W.K. Gleerup.

1873. Till algernes systematik .... Part 1, numbers i-iii. Lunds Universitets Arsskrift, 9:1-76.

1876. Species genera et ordines algarum .... Volume 3, part 1, Epicrisis systematis floridearum. viii +724 pages. Leipzig: Malnström.

1882. Till algernes systematik ... . Part 2, numbers iv-v. Lunds Universitets Ársskrift, 17:1-134+[2], 3 plates.

1883. Till algernes systematik ... . Part 3, number vi. Lunds Universitets Arsskrift, 19(2): 1-177+[4], 4 plates.

1885. Till algernes systematik ... . Part 4, number vii. Lunds Univer- sitets Arsskrift, $21(8): 1-117+[3], 1$ plate.

1887. Till algernes systematik.... Part 5, number viii. Lunds Universitets Arsskrift, 23:1-174, 5 plates.

1889. Species Sargassorun Australiae descriptae et dispositae. 133 pages, 31 plates. Stockholn: P.A. Norstedt \& Söner.

1892. Analecta algologica. Lunds Universitets Airsshrift, 28(6):1-182, 3 plates.

1896. Analecta algologica, continuatio III. Lunds Universitets Arsskrift, 33(9):1-106, 1 plate.

Allender, B.M., and G.T. Kraft

I983. The Marine Algae of Lord Howe Island (New South Wales): The Dictyotales and Cutleriales (Phaeophyta). Brunonia, 6:73-130.

Arasiki, $S$.

1964. How to Know the Seaweeds of Japan and Its Vicinity Fully Illustrated in Colours. $\mathrm{x}+217$ pages, $491+7+24$ figures. Tokyo: Hokuryukan. [In Japanese.]

Ardissone, $\mathrm{F}$

1871. Rivista dei Ceramii della flore Italiana. Nuovo Giornale Botanico Italiano, 3:32-50.

Areschoug, J.E.

1847. Phycearum, quae in maribus Scandinaviae crescunt, enumeratio, sectio prior. Societas Scientiarum Upsaliensis, Nova Acta, I3:223382.

1851. Phyceae capenses ... 32 pages. Uppsala: Reg. Acad. Typographus.

Ariga, $\mathrm{K}$.

1919. Taiwan kaiso saishu nisshi. [An Account of Taiwan Marine Algae Collections.] Transactions of the Natural History Society of Taiwan, 9:10-13.

1920. Taiwan oyobi Amonsan kaiso hikaku. [A Comparison of the Marine Algae of Taiwan and Amoy.] Transactions of the Natural History Society of Taiwan, 10:54-63.

1921. Kamayamashima no kaiso. [The Marine Algae of Turtle lsland.] Transactions of the Natural History Society of Taiwan, $11(53): 21$.

Barton, E.S.

1901. The Genus Halimeda. In M. Weber, editor, Siboga-Expeditie, Vitkomsten op Zoologisch, Botanisch, Oceanographisch en Geologisch Gebied ..., monograph LX:1-32, plates 1-4. Leiden: E.J. Brill.

Basson, P.W.

1979. Marine Algae of the Arabian Gulf Coast of Saudi Arabia (Second Half). Botanica Marina, 22(2):56-82.

B $\phi$ rgesen, F. [C.E.]

1905. Contributions à la connaissance du genre Siphonocladus Schmitz. Oversigt over det Kongelige Danske Videnskabernes Selskabs Forhandlinger, 3:259-29I.

1907. An Ecological and Systematic Account of the Caulerpas of the Danish West Indies. Det Kongelige Danske Videnskabernes Selskabs Skrifter Naturvidenskabelige og Mathematiske Afhandlinger, 7th series, $4(5): 337-392,31$ figures.

19I4. The Marine Algae of the Danish West Indies, Part 2: Phaeophyceae. Dansk Botanisk Arkiv, 2(2):1-66 + [2].

1920a. Marine Algae from Easter Island. In C. Skottsberg, editor, The Natural History of Juan Fernandez and Easter Island, 2:247-309. Uppsala.

1920b. The Marine Algae of the Danish West Indies, 111: Rhodophyceae, Part 6, with Addenda to the Chlorophyceae, Phaeophyceae and Rhodophyceae. Dansk Botanisk Arkiv, 3:369-498 + [6].

1932. A Revision of Forsskål's Algae Mentioned in Flora Aegyptiaco- 
Arabica and Found in His Herbariun in the Botanical Museun of the University of Copenhagen. Dansk Botanisk Arkiv, 8(2): 15 pages, 4 figures, 1 plate.

1933. Some Indian Rhodophyceae Especially from the Shores of the Presidency of Bombay. Bulletin of Miscellaneous Information (Royal Botanic Gardens, Kew), 3:1 13-142, 20 figures, 9 plates.

1934. Some Marine Algae from the Northern Part of the Arabian Sea with Remarks on Their Geographical Distribution. Det Kongelige Danske Videnskabernes Selskabs, Biologiske Meddelelser, I 1(6): 1-72, 8 figures, 2 plates.

1939. Marine Algae from the 1ranian Gulf Especially from the Innermost Part Near Bushire and the Island Kharg. In K. Jessen and R. Spärck, editors, Danish Scientific Investigations in Iran, part 1, pages 47-14I. Copenhagen: E. Munksgaard.

1942. Some Marine Algae from Mauritius, II1: Rhodophyceae, Part I: Porplyyridiales, Bangiales, Nemalionales. Det Kongelige Danske Videnskabernes Selskabs, Biologiske Meddelelser, 17(5):[1-3] + 4$63+[64]$, 27 figures, 2 plates.

Bory de Saint-Vincent, J. [B.G.M.]

1825. Dictionnaire classique d'Histoire naturelle. Volume 7 , iii +626 pages. Paris: Rey et Gravier.

1826. Dictionnaire classique d'Histoire naturelle. Volume 10, iii +642 pages. Paris: Rey et Gravier.

1827. Dictionnaire classique d'Histoire Naturelle. Volume 12, iii +634 pages. Paris: Rey et Gravier.

I 828. Cryptogamie. In M.M. D’Urville, Botanique. In L.1. Duperrey, Voyage autour $d u$ monde, exécuté par ordre du roi, sur la corvette de Sa Majesté, la Coquille, pendant les années I822, I823, I824 et 1825 , sous le Ministere et conformément avec instructions de S.G.M. le marquis Clermont Tonnerre, ministre de la marine ..., 2(fascicle 6):249-301. Paris: Arthus Bertrand.

Brand, F.

1904. Über die Anheftung der Cladophoraceen und über verschiedene polynesische Formen dieser Familie. Beihefte zum Botanischen Zentralblatt, 18:165-193.

Castagne, J.L.M.

1851. Catalogue des plantes qui croissent naturellement aux environs de Marseille, supplément. $\mathrm{i}+125$ pages, plates 8-11. Aix: Nicot et Pardigon.

Chang, C.F., and Xia Bangmei

1976. Studies on Chinese Species of Gracilaria. Studia Marina Sinica, 11 (I 2):9I-163, plates I, 2.

Chen, C., and Y.M. Chiang

1982. Morphological Studies on the Family Helminthocladiaceae (Nemaliales, Rhodophycophyta) of Taiwan, II: Dermonema. Biological Bulletin, National Taiwan University, I7:95-10I.

Chen, C., Y.M. Chiang, and S.H. Tsai Chiang

1981. Morphological Studies on the Family Helminthocladiaceae (Nemaliales, Rhodophycophyta) of Taiwan, 1: Helminthocladia. Biological Bulletin, National Taiwan University, I6:27-32.

Chen, T.P.

1976. Culture of Gracilaria. In Aquaculture Practices in Taiwan, pages 145-149. Tapei, Taiwan: Fishing News Books, Ltd.

Chiang, Y.M.

1960. Marine Algae of Northern Taiwan (Cyanophyta, Chlorophyta, Phaeophyta). Taiwania, 7:5I-75.

1962a. Marine Algae of Northern Taiwan (Rhodophyta). Taiwania, 8: I 43-I65.

1962b. Marine Algae Collected from Penghu (Pescadores). Taiwania, 8:I67-180.

1970. Observations on the Development of the Carposporophyte of Scinaia pseudojaponica Yamada et Tanaka (Nemaliales, Chaetangiaceae). Journal of Phycology, 6(3):289-292, 10 figures.

1971. Observations on the Development of Spermatangia in Some Genera of the Helminthocladiaceae. Phycologia, 10:163-167.

1972. Observations on the Development of the Carposporophyte of
Liagora boergesenii Yamada (Nemaliales, Helminthocladiaceae). In K. Nisizawa, editor, Proceedings of the 7th International Seaweed Symposium, Sapporo, Japan, pages III-II3. New York: John Wiley \& Sons.

1973a. Notes on Marine Algae of Taiwan. Taiwania, 18:13-I 7.

1973b. Studies on the Marine Flora of Southern Taiwan. Bulletin of the Japanese Society of Phycology, 2 I:97-I 02.

1981. Cultivation of Gracilaria (Rhodophycophyta, Gigartinales) in Taiwan. In T. Levring, editor, Proceedings of the Xth International Seaweed Symposium, pages 569-574. New York: Walter de Gruyter \& Co.

1982. Cultivation of Porphyta in Taiwan. In R.T. Tsuda and Y.M. Chiang, editors, Proceedings of Republic of China-United States Cooperative Science Seminar on Cultivation and Utilization of Economic Algae, pages 81-86. Mangilao: University of Guam Marine Laboratory.

1985. Gracilaria from Taiwan: Key, List and Distribution of the Species. In 1.A. Abbott and J. N. Norris, editors, Taxonomy of Economic Seaweeds..., pages 8I-83. La Jolla, California: California Sea Grant College Program, University of California.

Chiang, Y.M., and C. Chen

1982. The Genus Liagora of Taiwan. Acta Oceanographie Taiwanica, 13:181-196.

1983. Studies on Dotyophycus yamadae (Ohmi et Itono) Abbott et Yoshizaki (Nemaliales, Rhodophycophyta) from Southern Taiwan. Japanese Journal of Phycology, 31:10-15.

Chiang, Y.M., and H.N. Chou

1980. The Occurrence of Zonaria stipitata on the Southern Coasts of Taiwan. Japanese Journal of Phycology, 28:165-167.

Chiang, Y.M., R. Huang, and H.M. Su

1974. The Uptake of ${ }^{65} \mathrm{Zn}$ by Marine Benthic Algae. Acta Oceanographie Taiwanica, 4:241-248.

Chihara, M.

1969 Pseudogloiophloea okamurai (Setchell) comb. nov. and Ishige sini cola (Setchell and Gardner) comb. nov. Bulletin of the Japanese Society of Phycology, I 7:I-4.

1970. Common Seaweeds of Japan in Color. 173 pages, 64 plates. Osaka: Hoikusha Publishing Co. Ltd.

1975. Marine Algae. 290 pages. Tokyo: Gakken Co., Ltd. [In Japanese.] Chou, H.N., and Y.M. Chiang

1976. Studies on Algin from Brown Algae of Taiwan, I: Estimation of Yield and Quality of Algin. Acta Oceanographie Taiwanica, 6:135I 39

1981. The Sargassum of Taiwan. Acta Oceanographie Taiwanica, 12:132-149.

Chou, R.C.Y.

1945. Pacific Species of Galaxaura, I: Asexual Types. Papers of the Michigan Academy of Science, Arts, and Letters, 30:33-55, plates I-1 I.

1947. Pacific Species of Galaxaura, 11; Sexual Types. Papers of the Michigan Academy of Science, Arts, and Letters, 31:3-24, plates 113.

Christensen, C.F.A.

1905. Index Filicum . . . 384 pages. Copenhagen: H. Hagerup.

1906. Index Filicum . . . pages i-ix $+385-744+$ xiii-lix. Copenhagen: H. Hagerup.

Chueh, C.T., and C.C. Chen

1982. Seaweed Economics in Taiwan. In R.T. Tsuda and Y.M. Chiang, editors, Cultivation and Utilization of Economic Algae, pages 9-I6. Mangilao, Guam: University of Guam Marine Laboratory.

Collins, F.S.

1909. The Green Algae of North America. Tufts College Studies, Scientific Series, 2:79-480, I 8 plates.

Cotton, $\Lambda . D$

1914. The Japanese Seaweed, Tosika nori. Bulletin of Miscellaneous Information (Royal Gardens, Kew), 19I4:2I9-222. 
1915. Some Chinese Marine Algae. Bulletin of Miscellaneous Information (Royal Gardens, Kew), 1915:107-113.

Dawson, E.Y.

1950. Notes on Some Pacific Mexican Dictyotaceae. Bulletin of the Torrey Botanical Club, 77:83-93.

1953. Marine Red Algae of Pacific Mexico, Part I: Bangiales to Corallinaceae subf. Corallinoideae. Allan Hancock Pacific Expeditions, 17(1): 1-239.

1954a. The Marine Flora of Isla San Benedicto Following the Volcanic Eruption of 1952-53. Allan Hancock Foundation Publications, Occasional Papers, 16:1-13.

1954b. Notes on Tropical Pacific Marine Algae. Bulletin of the Southern California Academy of Sciences, 53:1-7.

1954c. Marine Plants in the Vicinity of Nha Trang, Viêt Nam. Pacific Science, 8:373-481.

1962. New Taxa of Benthic Green, Brown and Red Algae. 105 pages. Santa Ynez, California: Beaudette Foundation for Biological Research.

Decaisne, J.

1841. Plantes de l'Arabie heureuse, recueuillies par M.P.E. Botta. Archives du Museum National d'IIistoire Naturelle (Paris), 2:89-199.

1842. Mémoire sur les Corallines ou Polypiers Calcifères. Annales des Sciences Naturelles, Botanical series, 1I, 18:96-128.

Delile, A.R.

1813. Flore de l'Egypte: Explication des planches. In France (Commission d'Egypte), Description de l'Egypte ou recueil des observations et des recherches qui été faites en Egypte pendant l'expédition de l'armée Francaise (1798-1801). Histoire Naturelle de Paris, 2(4):i + 145-320, plates 1-62. [Plates published in 1826.]

De Toni, G.B.

1895. Phyceae japonicae novae addita enumeratione algarum in ditione maritima japoniae hucusque collectarum: Alghe marine del Giappone ed isole ad esso appartenenti con illustrazione di alcune specie nuove (con 2 tavole). Memorie del Real Istituto Veneto di Sciienze, Letere ed Arti, 25(5):1-78, plates 1, 2.

1905. Sylloge algarum omnium hucusque cognitarum. Volume 4, section 4: Sylloge Floridearum ... Pages $[\mathrm{v}]+$ 1523-1973. Padovd: Typis Seminarii.

1924. Sylloge algarum omnium hucusque cognitarum. Volume 6: Sylloge Floridearum ... sectio 5: additamenta. xi +767 pages. Padova: Typis Seminarii.

Dickie, G.

1874. On the Algae of Mauritius. The Journal of the Linnean Society (Botany), 14:190-202.

Dixon, P.S.

1967. The Typification of Fucus cartilagineus L. and F. corneus Huds. Blumea, 15:55-62.

Doty, M.S.

1983. Coral Reef Diversified Farming. In C.K. Tseng, editor, Proceedings of the Joint China-U.S. Phycology Symposium, pages 437-478. Beijing, Chind: Science Press.

Doty, M.S., and I.A. Abbott

1964. Studies in the Helminthocladiaceae, 111: Liagoropsis. Pacific Science, 18:441-452.

Doty, M.S., and E.G. Meñez

1960. Tiffaniella, a New Genus in the Ceramiales. Transactions of the American Microscopical Society, 79:135-144.

Doty, M.S., and J.N. Norris

1985. Eucheuma Species (Solieriaceae, Rhodophyta) That Are Major Sources of Carrageenan. In I.A. Abbott and J.N. Norris, editors, Taxonomy of Economic Seaweeds ... , pages 47-61. La Jolla, California: California Sea Grant College Program, University of California, La Jolla.

Drew, K.M.

1956. Conferva ceramicola Lyngbye. Botanisk Tidsskrift, 53:67-74.
Drew, K.M., and R. Ross

1965. Some Generic Names in the Bangiophyceae. Taxon, 14:93-99. Ducker, S.C.

1967. The Genus Chlorodesmis (Chlorophyta) in the Indo-Pacific Region. Nova Hedwigia, 13:145-182, plates 26-43.

Ducker, S.C., W.T. Williams, and G.N. Lance

1965. Numerical Classification of the Pacific Forms of Chlorodesmis (Chlorophyta). Australian Journal of Botany, 13:489-499.

Ducluzeau, J.A.P.

1805. Fssai sur l'histoire naturelle des conferves des environs de Montpellier. $\mathrm{i}+89$ [90-92 + presentation] pages. Montpellier: Auguste Ricard.

Egerod, L.E.

1952. An Analysis of the Siphonous Chlorophycophyta with Special Reference to the Siphonocladales, Siphonales, and Dasycladales of Hawaii. University of California Publications in Botany, 25(5):325-454, 13 plates.

Eubank, L.L.

1946. Hawaiian Representatives of the Genus Caulerpa. University of California Publications in Botany, 18(18):409-432, I plate.

Falhenberg, P.

1901. Die Rhodomelaceen des Golfes Neapel und der angrenzenden Meeres-Abschnitte. Fauna und Flora des Golfes von Neapel und der angrenzenden Meeresteile. Volume $26, \mathrm{xvi}+754$ pages, 10 figures, 24 plates. Berlin: R. Friedlander \& Sohns.

Fan, K.C.

1951. The Genera Gelidium and Pterocladia of Taiwan. Taiwan Fisheries Research Institute, Laboratory of Biology Report, 2:1-22, 5 plates.

1952. The Structure, Methods of Branching and Tetrasporangid Formation of Caloglossa. Taiwan Fisheries Research Institute, Hydrobiology Report, 4:1-16.

1953a. A List of Edible Sea-weeds in Taiwan. Taiwan Fisheries Research Institute, Hydrobiology Report, 5/6:1-11.

1953b. The 1dentity of Dilophus radicans Okamura. Taiwan Fisheries Research Institute, Hydrobiology Report, 5/6:12-13.

1962. Studies on the Reproductive Organs of Red Algae, 11: The Genus Dermonema. Acta Botanica Sinica, 10:336-340.

1974. Notes on Algal Taxonomy, 1: A Review of Certain New Taxa on the Marine Algae of Taiwan Province, China. Acta Phytotaxonomica Sinica, 12:249-255.

Fan, K.C., and Y.C. Wang

1974. Studies on the Marine Algae of the Hsisha lslands, China, 1: New Species and New Records of the Family Nemalionaceae (Rhodophyta). Acta Phytotaxonomica Sinica, 12:489-495.

Farr, E.R., J.A. Leussink, and F.A. Stafleu

1979. Index nominum genericorum (plantarum). V'olume 1: Aa-Epochnium, xxvi +630 pages; volume 1I: Eprolithus-Peersia, pages 631 1276; volume 111: Pegaeophyton-Zyzygium, pages 1277-1896. Utrecht: Bohn, Scheltema \& Holkema.

Feldmann, J.

1938. Sur la classification de l'ordre des Siphonocladals. La Revue Générale de Botanique, 50:571-597.

Feldmann, J., and G. Himel

1934. Observations sur quelques Gélidiacées. Revue Générale de Botanique, 46:528-549.

Feldmann-Mazover, G.

1941. Recherches sur les Céramiacées de la Méditerranée occidentale. ii + 510 pages, 4 plates. Alger: Imprimerie Minerva

Forsshål, P.

1775. Flora Aegyptiaco-Arabica ... [1]-32 + i-cxxvi + [1] + [1]-219+ $[220]$ pages + frontispiece + map. Copenhagen: Moller.

Foslie, M.H.

1900. Revised Systematical Survey of the Melobesieae. Det Kongelige Norske Videnskabers Selskabs Skrifter, 1900(5):1-22. 
1905. Remarks on Northern Lithothamnia. Det Kongelige Norske Videnskabers Selskabs Skrifter, 1905(3): I-I38.

Gaillon, F.B.

1828. Résumé méthodique des classifications des thalassiophytes ... In F. Cuvier, Dictionnaire des sciences naturelles ... second edition, 53:1-59, plates 1, 2. Strasbourg: F.G. Levrault.

Gardner, N.L.

1909. New Chlorophyceae from California. University of California Publications in Botany, 3:371-375, 1 plate.

Gepp. A., and E.S. Gepp

1908. Marine Algae (Chlorophyceae and Phaeophyceae) and Marine Phanerogams of the "Sealark" Expedition, Collected by J. Stanley Gardiner .... Transactions of the Linnean Society, 7:163-188.

Gray, J.E.

1866. On Anadyomene and Microdictyon, with the Description of Three New Allied Genera, Discovered by Menzies in the Gulf of Mexico. Journal of Botany, British and Foreign, 4:41-51, 65-72.

Gray. S.F.

1821. A Natural Arrangement of British Plants.... Volume I, xxviii + 824 pages, 21 plates. London.

Greville, R.K.

1830. Algae Brittannicae, or Descriptions of the Marine and Other Articulated Plants of the British Islands, Belonging to the Order Algae. Ixxxviii + 2I8 pages, 19 plates. Edinburgh: Maclachlan and Stewart.

Hamel, 6 .

1939. Pheophycées de France. Part 5, pages i-xlvii, 337-432, 10 plates. Paris.

Hansen, J.E., J.E. Packard, and W.T.Dorle

1981. Mariculture of Red Seaureds. $\mathrm{x}+42$ pages. La Jolla, California: California Sea Grant College Program, University of California, La Jolla.

Hariot, P.A.

1889. Algues. Mission Scientifique du Cap Horn, I882-1883. Volume 5(Botanique), pages 3-1 19, plates I-9. Paris: Gauthier-Villars et fils.

Harvey, W.H.

1847-1849a [1849]. Phycologia brittannica .... Volume 2, vi + plates 12 I -240 with text [ 1847 , fascicles $21-24$ comprising plates $121-$ 144 with text; I848a, fascicles $25-36$ comprising plates $145-216$ with text; 1849a, fascicles $37-40$ comprising plates $217-240$ with text]. London: Reeve, Benham \& Reeve.

1848b [1847]. Nereis australis .... Part I, [iii] + viii + 64 pages, plates 1-25. London: Reeve Brothers.

1849b. Nereis australis... Part 11, pages 65-124, plates 26-50. London: Reeve Brothers.

1853. Nereis boreali-americana ..., Part II: Rhodospermeae. First issue, ii + 258 pages, plates 13-26. Washington, D.C.: Smithsonian lnstitution and London: John Van Voorst. [Third issue, 1858 (dated 1853), published as Smithsonian Contributions to Knowledge, $5(5): i i+258$ pages, plates $13-36$.]

1854. Some Account of the Marine Botany of the Colony of Western Australia. Transactions of the Royal Irish Academy, 22:525-566.

1856. Algae of Japan. In A.S. Gray, Account of the Botanical Specimens. In Narrative of the Expedition of an American Squadron to the China Seas and Japan..., 2:331-332. Washington: Beverley Tucker. [Listed under "Commodore M.C. Perry" in most libraries.]

1858. Nereis boreali-americana ..., Part III: Chlorospermeae. First issue, [ii] + 140 pages, plates 37-50. Washington, D.C.: Smithsonian Institution and London: John van Voorst. [Also issued as Smithsonian Contributions to Knowledge, 10:1-140, plates 1-14.]

1859. Characters of New Algae, Chiefly from Japan and Adjacent Regions, Collected by Charles Wright in the North Pacific Ex- ploring Expedition under Captain John Rodgers. Proceedings of the American Academy, 4:327-335.

1863. Phycologia Australica .... Volume 5, vi + plates 241-300 with text, index [rii]-x, and synoptic catalogue [v]-lxxiii. London: Lovell Reeve.

Hauch, F.

1884. Die Meeresalgen Deutschlands und Österreichs. In L. Rabenhorst, Kryptogamen-Flora von Deutschland, Oesterreich und der Schweiz, edition 2, 2:321-512. Leipzig: Edward Kummer.

1886. Über einige von J.M. Hildenbrandt in Rothen Meere und in Indischen Ocean gesammelte Algen, I. Hedwigia, 25(5):165-168.

I 887. Über einige von J.M. Hildebrandt in Rothem Meere und in Indischen Ocean gesammelte Algen, IV. Hedwigia, 26:41-45.

Heydrich, F.

1894. Bietrage zur kenntniss der Algenflora von Ost-Asien besonders der Insel Formosa, Mollukken und Liukiu-Inseln. Hedwigia, 33:267-306. 2 plates.

Higashi, M.

1934. Natural Coloured Figures and Descriptions of the Japanese Seaweeds. Pages i, 1-4, 1-5, 1-8, 1-80, 80 plates. Tokyo.

Holmes, E.M.

I 896 [1895]. New Marine Algae from Japan. Journal of the Linnaean Society of Botany, 31:248-269, 12 plates.

Hommersand, M.H.

1963. The Morphology and Classification of Some Ceramiaceae and Rhodomelaceae. University of California Publications in Botany, 35(2): 165-366.

Hooker, W.J.

1833. Class XXIV: Cryptogamia. The English Flora of Sir James Edward Smith. Volume 5, pages 322-385. London.

Hooker, W.J., and G.A. Arnott

1838. The Botany of Captain Beechey's Voyage. Part 6, pages 24I-288, plates 50-59. London: Henry G. Bohn.

Horikawa, Y.

1919. Taiwan kaiso. [Marine Algae of Taiwan.] Transactions of the Natural History Society Formosa, 9:13-16.

Howe, M.A.

1905. Phycological Studies, II: New Chlorophyceae, New Rhodophyceae, and Miscellaneous Notes. Bulletin of the Torrey Botanical Club, 32:563-586, 6 plates.

19I4. The Marine Algae of Peru. Memoirs of the Torrey Botanical Club, 15:[i] + 185 pages, 66 plates.

1917. A Note on the Structural Dimorphism of Sexual and Tetrasporic Plants of Galaxaura obtusata. Bulletin of the Torrey Botanical Club, 43:62I-624.

1918. Further Notes on the Structural Dimorphism of Sexual and Tetrasporic Plants in the Genus Galaxaura. Brooklyn Botanical Garden Memoir, 1:191-197.

1920. Algae. In N.L. Britton and C.F. Millspaugh, The Bahama Flora, pages 553-618. New York: Published by the authors.

Itono, $\mathrm{H}$.

1972. The Genus Ceramium (Ceramiaceae, Rhodophyta) in Southern Japan. Botanica Marina, 15:74-86.

1973. Notes on Marine Algae from Hateruma Island, Ryukyu. Bota nical Magazine (Tokyo), 86:155-168.

1977 a. Studies on the Southern Japanese Species of Galaxaura (Rhodophyta). Micronesica, 13:1-26.

1977b. Studies on the Ceramiaceous Algae (Rhodophyta) from Southern Parts of Japan. Bibliotheca Phycologica, 35: 499 pages. Vaduz: J. Cramer.

Jackson, B.D.

1893. Index Kewensis . . . xvi +728 + vii $+729-1268$ pages. Oxford: Clarendon Press. 
Kjellman, F.R.

1889. Om Beringhafvets algflora. Det Kongelige Vetenskaps Academiens Handlingar, 23(8): 58 pages.

1897a. Japanska arter af slägtet Porphyra. Bihang till Kongliga Svenska Vetenskaps-Akademiens Handlingar, 23(4):1-34.

1897b. Marine Chlorophyceer från Japan. Bihang till Kongliga Svenska Vetenskaps-Akademiens Handlingar, 23(11):1-44, 4 plates.

1900. On Floridé-Slägtet Galaxaura dess organografi och systematik. Bihang till Kongliga Svenska Vetenskaps-Akademiens Handlingar, $33(1): 1-107,20$ plates.

Kraft, G.T

1972. Preliminary Studies of Philippine Eucheuma Species (Rhodophyta), Part 1: Taxonomy and Ecology of Eucheuma arnoldii Weber-van Bosse. Pacific Science, 26:318-334.

1981. Rhodophyta: Morphology and Classification. In C.S. Loban and M.J. Wymne, editors, The Biology of Seaweeds, pages 6-51. Berkeley: University of California Press.

Krauss, C.F.F. von

1846. Algae. In Beiträge zur Flora des Cap-und Natallandes . ., 29:192215.

Ḱunt/e, C.E.O.

1898. Revisio generum plantarum... Volume 3, part 3, pages 1-576. Leiprig: Arthur Felix

Kützing, F.T.

1843. Phycologia generalis odor Anatomie, Physiologie und Systemkunde der Tange. . . xxxii +458 pages, 80 plates. Leipzig: F.A. Brockhaus.

1845. Phycologia Germanica: Deutschlands Algen in bündigen Beschreibungen. vii +240 pages. Nordhausen: Wilh. Köhne.

1847. Diagnosen einiger neuen auständischen Algenspecies .... Flora oder Allgemeine Botanische Zeitung, 30(48):773-776.

1849. Species algarum .... vi +922 pages. Leiprig: F.A. Brockhaus.

1856. Tabulae phycologicae oder Abbildungen der Tange. Volume 6 , vi + 35 pages, 100 plates. Nordhausen: Kosten des Verfassers.

1858. Tabulae phycologicae oder Abbildungen der Tange. Volume 8, parts $1-2$, ii +48 pages, 100 plates. Nordhausen: Kosten des Verfassers.

1860. Tabulae phycologicae oder Abbildungen der Tange. Volume 10, parts $1-2$, iv +39 pages, 100 plates. Nordhausen: Kosten des Verfassers.

1866. Tabulae phycologicae oder Abbildungen der Tange. Volume 16, parts $1-2, i+35$ pages, 100 plates. Nordhausen: Kosten des Verfassers.

1868. Tabulae phycologicae oder Abbildungen der Tange. Volume 18, parts $1-2, j+35$ pages, 100 plates. Nordhausen: Kosten des Verfassers.

1869. Tabulae phycologicae oder Abbildungen der Tange. Volume 19, parts 1-2, 36 pages, 100 plates. Nordhausen: Kosten des Verfassers.

Kylin, $\mathrm{H}$.

1928. Entwicklungsgeschichtliche Florideenstudien. Lunds Universitets Arsskrift, 24(4): 1-127.

1931. Die Florideenordnung Rhodymeniales. Lunds Universitets Arsskrift, 27(11): 1-48, 20 plates.

1956. Die Gattungen der Rhodophyceen. xv +673 pages. Lund: C.W.K. Gleerups

L.amouroux, J.V.F.

1809a. Exposition des caracteres du genre Dictyota (1), et tableau des especes qu'il renferme. Journal de Botanique (Paris), 2:38-44.

$1809 \mathrm{~b}$. Observations sur la physiologie des algues marines, et description de cinq nouveaux genres de cette famille. Nouveau Bulletin des Sciences de la Société Philomatique de Paris, 1:330-333.

1812. Fxtrait d'un memoire sur la classification des polypiers coralligènes non entièrement pierreux. Nouveau Bulletin des Sciences de la Société Philomatique de Paris, 3:181-188.

1813. Fssai sur les genres de la famille des thalassiophytes non articulés.
Annales du Muséum National d'Histoire Naturelle de Paris, 20:2147, 115-139, 267-296, 5 plates.

1816. Histoire des polypiers coralligènes flexibles, le vulgairement nommés Zoophytes. Ixxxiv +560 pages, 19 plates. Caen: F. Poisson.

Le Jolis, A.

1863. Liste des algues marines de Cherbourg. 168 pages, 6 plates. Paris: J.B. Baillière et Fils. [Preprinted from Mémoires de la Societé Imperiales Nationale des Sciences Naturelles et Mathematiques de Cherbourg, 1864, 10:5-168.]

lewis, S.M., J.N. Norris, and R.B. Searles

1987. The Regulation of Morphological Plasticity in Tropical Reef Algae by Herbivory. Ecology, 68.

Liaw, J.P., and Y.M. Chiang

1979. Culture Studies on the Concocelis of Porphyra angusta Udea. Journal of the Fisheries Society of Taiwan, 6:59-65.

Linnaeus, C.

1753. Species plantarum, exhibentes plantas rite cognitas, ad genera relatas, cum differentiis specificis, nominibus trivialibus, synonymis selectis, locis natalibus, secundum systema sexuale digestas. Volume 2, pages 561-1200. Stockholm: 1mpensis Laurentii Salvii.

Liu, T.Y

1982. Quality Improvement of Algin from Sargassum. In R.T. Tsuda and Y.M. Chiang, editors, Proceedings of Republic of China-United States Cooperative Science Seminar on Cultivation and Utilization of Economic Algae, pages 65-80. Mangilao: University of Guam Marine Laboratory.

Lobban, C.S., and M.J. Wynne

1981. The Biology of Seaweeds. xi +786 pages. Berkeley: University of California Press.

Martens, G. von

1866. Die preussische Expedition nach Ost-Asiens, Botanischer Theil. Die Tange. 152 pages, plates 1-8. Berlin: Ober-hofbuchdruckerei, R.V. Decker.

Marover, C.

1938. Les Céramiaceés de l'Afrique dur Nord. Bulletin de la Société d'Histoire Naturelle de l'Afrique due Nord, 29: 317-331.

$\mathrm{Mat/a}, \mathrm{A}$.

1907. Saggio di algologia oceanica ... Nuova Notarisia, Rassegna Consacrata allo Studio della Alghe, 18:126-152.

Meneghini, G.

1840. Lettera del Prof. Giuseppe Meneghini al Dottora Iacob Corinaldi a Pisa. ${ }^{[4]}$ pages. Padova: Tipografia Prosperi.

Michaneh, $G$

1978. Trends in Applied Phycology, with a Literature Review: Seaweed Farming on an lndustrial Scale. Botanica Marina, 21:469-475.

Mikami, H.

1965. A Systematic Study of the Phyllophoraceae and Gigartinaceae from Japan and lts Vicinity. Scientific Papers of the Institute of Algological Research, Faculty of Science, Hokkaido University, 5:181285, plates $1-\mathrm{X} 1$.

Montagne, J.P.F.C.

1838. De l'organisation et du mode de reproduction des Caulerpées et en particulie:- du Caulerpa webbiana, espèce nouvelle des îles Canaries. Annales des Sciences Naturelles, 2nd series, 9:129-150. Paris.

1842. Troisième centurie de cellulaires exotiques nouvelles ....Annales des Sciences Naturelles, Botanique, 18:241-282.

1844. Quelques observations touchant la structure et la fructification des genres Ctenodus, Kütz., Delisea, Lamx., et Lenormandia, Montag., de la famille des Floridées. Annales des Sciences Naturelles, Botanique, series 3,1:126-129, 151 -161, plates 10, 11 .

1846. Plhyceae. In M.C. Durieu de Maisonneuve, Flore d'Algérie, Botanique, 1: Cryptogamie. In Exploration scientifique de l'Algérie ..., pages 1-197, plates 1-16. Paris: Imprimerie Royal. 
1849. Sicieme Centurie de Plantes cellulaires nouvelles, tant indigenes qu'exotiques, Decades 111. a VI. Annales des Sciences Naturelles, 11:33-66.

1850. Pugillus Algarum Yemensium .... Annales des Sciences Naturelles, 13:236-248.

Murray, G.

1889. On Boodlea, a New Genus of Siphonocladaceae. Journal of the Linnean Society, Botany, 25:243-245.

Nakamura, $Y$.

1950. New Ceramiums and Campylaephoras from Japan. Science Papers, Institute of Algal Research, Hokkaido University Faculty of Science, 3:155-172.

1965. Species of the Genera Ceramium and Campylaephora, Especially Those of Northern Japan. Science Papers, Institute of Algal Resea rch, Hokkaido University Faculty of Science, 5:1 19-180.

Needham, J.

1970. Clerks and Craftsmen in China and the W'est. 470 pages. Cambridge: Cambridge University Press.

Nees von Esenback, C.

1820. Horae physicae berolinenses.... Part 1, pages 1-7, plate 1. Bonn: Adolphi Marcus.

Nelson, S.G., S.S. Yang, C.Y. Wang, and Y.M. Chiang

1983. Yield and Quality of Agar from Species of Gracilaria (Rhodophyta) Collected from Taiwan and Micronesia. Botanica Marina, 26:36l-366

Nicholson, D.H., and J.N. Norris

1983. Ordinal Orthography: Nemalionales vs. Nemaliales (Rhodophyta). Taxon, 32(2):288-292.

Nizamuddin, $M$.

1970. Phytogeography of the Fucales and Their Seasonal Growth. Botanica Marina, 12:131-139.

Norris, J.N., and K.E. Bucher

1982. Marine Algae and Seagrasses from Carrie Bow Cay, Belize. In K. Rützler and 1.G. Macintyre, editors, The Atlantic Barrier Reef Ecosystem at Carrie Bow Cay, Belize, 1: Structure and Communities. Smithsonian Contributions to the Marine Sciences, 12:167223.

Norris, J.N., and W.H. Fenical

1985. Natural Products Chemistry: Uses in Ecology and Systematics. In M.M. Littler and D.S. Littler, editors, Handbook of Phycological Methods, V: Ecological Field Methods for Macroalgae, pages 121 145. New York/Cambridge: Cambridge University Press.

Norris, J.N., and H.W. Johansen

1981. The Articulated Coralline Algae of the Gulf of California, Mexico, 1: Amphiroa Lamouroux. Smithsonian Contributions to the Marine Sciences, 9:1-29.

Nozawa, Y.

1963. Systematic Anatomy of the Genus Rhodopeltis. Memoirs of the Janshin Junior College, 5:1-48, 2 plates.

1970. Systematic Anatomy of the Red Algal Genus Rhodopeltis. Pacific Science, 24:99-133.

Ohmi, H.

1958. The Species of Gracilaria and Gracilariopsis from Japan and Adjacent Waters. Memoirs of the Faculty of Fisheries, Hokkaido University, 6(1): 1-66.

Okamura, K.

1895. New or Little Known Algae from Japan. The Botanical Magazine (Tokyo), 9:445-455, 472-482, 1 plate.

1896. Contributions to the Knowledge of the Marine Algae of Japan, 11. The Botanical Magazine (Tokyo), 10:23-36.

1897. On the Algae from Ogasawarajima (Bonin 1slands). The Botanical Magazine (Tokyo), 11:1-17, 1 plate, 4 figures.

1899. Contributions to the Knowledge of the Marine Algae of Japan, 111. The Botanical Magazine (Tokyo), 13:2-10, 35-43, 11 plates.
1901. Illustrations of the Marine Algae of Japan. Volume 1, part 5, pages 57-74 [Japanese text, pages 79-102], 5 plates. Tokyo: Keigyosha \&. Co.

1902. Illustrations of the Marine Algae of Japan. Volume 1, part 6, pages 75-93 [Japanese text, pages 103-128], 5 plates. Tokyo: Keigyosha \&: Co.

1907. Icones of Japanese Algae. Volume 1, pages 1-119, plates 1-25. Tokvo: Kazamashobo.

1908. Icones of Japanese Algae. Volume 1, pages 121-233, plates 2645. Tokvo: Kazamashobo.

1909. Icones of Japanese Algae. Volume 2, pages 1-62, plates 51-65. Tokyo: Kazamashobo.

1910. Icones of Japanese Algae. Volume 2, pages 63-126, plates 66-85. Tokyo: Kazamashobo.

1912. Illustrations of the Marine Algae of Japan. Volume 2, pages $127-$ 191, plates 86-100. Tokyo: Kazamashobo.

1913. Icones of Japanese Algae. Volume 3, pages 1-77, plates 101-120. Tokyo: Kazamashobo.

1914. Icones of Japanese Algae. Volume 3, pages 79-121, plates 121130. Tokyo: Kazamashobo.

1915a. Icones of Japanese Algae. Volume 3, pages 123-218, plates 131150. Tokyo: Kazamashobo.

19l5b. Taiwan no ten gusa. [Gelidium of Taiwan.] Shokubutsugaku Zasshi, 29:57-58. [ln Japanese.]

1916. List of Marine Algae Collected in the Caroline and Mariana lslands. The Botanical Magazine (Tokyo), 30:1-14.

1919. Notes on Some Marine Algae from Amoy, 1. The Botanical Magazine (Tokyo), 33:12-14.

1921. Icones of Japanese Algae. Volume 4, pages 63-149, plates $166-$ 185. Tokyo: Kazamashobo.

1923. Icones of Japanese Algae. Volume 4, pages 185-205, plates 196200. Tokyo: Kazamashobo.

1930. On the Algae from the lsland Hatidyo. Records of Oceanographic Works in Japan, 2(2):92-110, 2 plates.

1931. On the Marine Algae from Kôtôsho (Botel Tobago). Bulletin of the Biogeographical Society of Japan, 2(2):95-122, 3 plates.

1932. Icones of Japanese Algae. Volume 6, pages 49-101 [Japanese text, 47-96], plates 276-300. Tokyo: Kazamashobo.

1934a. On Gelidium and Pterocladia of Japan. Journal of the Imperial Fisheries Institute, 29(2):47-67, 18 plates.

1934b. Icones of Japanese Algae. Volume 7, pages 9-37 [Japanese text, 9-34], plates 306-320. Tokvo: Kazamashobo.

1935a. Icones of Japanese Algae. Volume 7, pages 39-72 [Japanese text 35-63], plates 321-340. Tokyo: Kazamashobo.

1935b. Taiwan-san Tengusa ni Tsuite. Japanese Association for the Advancement of Science, 10:441-443.

1936. Nippon Kaiso-shi. [Marine Algae of Japan.] Frontispiece $+9+6$ $+964+11$ pages. Tokyo: Published by the author.

1937. Icones of Japanese Algae. Volume 7, pages 73-79, plates 341345. Tokyo: Kazanashobo.

Oshima, $M$.

1915. Taiwan san Tengusa. [Gelidium in Taiwan.] Transactions of the Natural History Society of Taiwan, 5:55.

Papenfuss, G.F

1944. Notes on Algal Nomenclature, 111: Miscellaneous Species of Chlorophyceae, Phaeophyceae and Rhodophyceae. Farlowia, 1(3):337-346

1950. Review of the Genera of Algae Described by Stackhouse. Hydrobiologia, 2:181-208.

1956. Notes on South African Marine Algae, 1V. The Journal of South African Botany, 22(2):65-77.

1960. On the Genera of the Ulvales and the Status of the Order. Journal of the Linnean Society, Botany, 56:303-318.

1967. Notes on Algal Nomenclature, V: Various Chlorophyceae and 
Rhodophyceae. Phykos, 5:95-105.

1977. Review of the Genera of Dictyotales (Phaeophycophyta). Bulletin of the Japanese Society of Phycology, 25(supplement):271-287.

Papenfuss, G.F., K.E. Mshigeni, and Y.M. Chiang

1982. Revision of the Red Algal Genus Galaxaura with Special Reference to the Species Occurring in the Western Indian Ocean. Botanica Marina, 25:401-444.

Piccone, $\mathrm{A}$.

1884. Alghe. In Crociera del Corsaro alle Isole Madera e Canarie del Capitano Enrico d'Albertis, 60 pages. Genova: R. Instituto SordoMuti.

Pilger, R.

1911. Die Meeresalgen von Kamerun, nach der Sammlung von C. Ledermann. Sonderabdruck aus Engler's Botanischen Jahrbüchern, 46(3):294-323, 26 figures.

1920. Algae Mildbraedianae Annobonenses. Botanische Jahrbücher für Systematik, Pflanzengeschichte und Pflanzengeographie Herausgege-

Reinbold, T ben von A. Engler, 57(1):1-14, 34 figures.

1901. The Marine Algae. In J. Schmidt, Flora of Koh Chang: Contributions to the Knowledge of the Vegetation in the Gulf of Siam, Part IV. Botanisk Tidsskrift, 24(2):187-201.

1905. Einige neue Chlorophyceen aus dem lnd. Ocean (Niederl. Indien), gesammelt von A. Weber van Bosse. La Nuova Notarisia, 16:145-149.

Rho, J.H.

1958. A Preliminary Survey of the Marine Algae of Korea. Collectio Theseon, Scienta Naturalis, Universitas Sung Kyun Kwan, 3:41143.

Ryther, J.H.

1979. Aquaculture in China. Oceanus, 22:21-28.

Saito, Y., and H.B.S. Womersley

1974. The Southern Australian Species of Laurencia (Ceramiales: Rhodophyta). Australian Journal of Botany, 22:815-874.

Santelices, B., and J.G. Stewart

1985. Pacific Species of Gelidium Lamouroux and Other Gelidiales (Rhodophyta), with Keys and Descriptions to the Common or Fcononnically lmportant Species. In 1.A. Abbott and J.N. Norris, editors, Taxonomy of Economir Seaweeds ..., pages 17-31. La Jolla, California: California Sea Grant College Program, University of California, La Jolla.

Schmit, F.

1893. Die Gattung Lophothalia J. Ag. Sonderabdruck aus den Berichten er Deutschen Botanischen Gesellschaft, 11(3):212-232.

1894. Neue japonische Florideen von K. Okamura. Hedwigia, 33:190201, 1 plate

1895. Marine Florideen von Deutsch-Ostafrika. In A. Engler, Beiträge zur Flora von Afrika. Botanische Jahrbücher führ Systematik, Pflanzengeschichte und Pflanzengeographie, 221:137-177.

Sclimit?, F, and P. Hauptfleisch

1897. Rhodymeniaceae. In H.G.A. Engler and K.A.E. Prantl, Die natürlichen Pflanzenfamilien . ., 1, 2(149-I50):396-405, 4 figures. Leipzig: Wilhelm Engelmann.

Segawa, $S$

1941. Systematic Anatomy of the Articulated Corallines (V1). The Journal of Japanese Botany, 17(8):450-456, 4 figures.

1974. Coloured Illustrations of the Seaveeds of Japan [Gen shoku Nihon Kaiso Zukan]. 175 pages, 72 figures +12 plates. Tokyo: Hoikusha Publ. Co.

Segi, T.

1951. Systematic Study of the Genus Polysiphonia from Japan and lts Vicinity. Journal of the Faculty of Fisheries, Prefectural University, Mie, 1(2): 169-272.

Setchell, W.A.

1914. The Scinaia Assemblage. University of California Publications in Botany, 6:79-152, 6 plates.
1924. American Samoa, Part I: Vegetation of Tutuila Island. Carnegie Institution of Washington Publication, 20:i-vi + 1-275 [Algae, 28$46 ; 55-57$ ], 37 plates.

1925. Notes on Microdictyon. University of California Publications in Botany, 13:101-107.

1926. Tahitian Algae Collected by W.A. Setchell, C.B. Setchell, and H.E. Parks. University of California Publications in Botany, 12(5):61-142, 16 plates.

1931. Hong Kong Seaweeds, 1I. Hong Kong Naturalist, 2(4):237-253.

1943. Mastophora and the Mastophoreae: Genus and Subfamily of the Corallinaceae. Proceedings of the National Academy of Sciences, 29(5): $127-135$.

Setchell, W.A., and N.L. Gardner

1920. Phycological Contributions, I. University of California Publications in Botany, 7:279-324, 11 plates.

Shang, Y.C.

1976. Economic Aspects of Gracilaria Culture in Taiwan. Aquaculture, $8: 1-7$

Sheath, R.G., and K.M. Cole

1984. Systenatics of Bangia (Rhodophyta) in North America, I: Biogeographic Trends in Morphology. Phycologia, 23:383-396.

Shen, Y.F., and K.C. Fan

1950. Marine Algae of Formosa. Taiwania, 1(2-4):3 I7-345.

Silva, P.C.

1952. A Review of Nomenclatural Conservation in the Algae from the Point of View of the Type Method. University of California Publications in Botany, 25(4):241-324.

Solier, A.J.J

1847. Sur deux algues zoosporées formant le nouveau genre Derbesia. Annales des Sciences Naturelles (Botany), 7:157-166.

Solms-Laubach, $\mathrm{H}$.

1893. Über die Algengenera Cymopolia, Neomeris und Bornetella. Annales du Jardin Botanique de Buitenzorg, 11:61-97, 2 plates.

1895. Monograph of the Acetabularieae. Transactions of the Linnean Society of London, 2nd series, Botany, 2:1-39.

Sonder, O.G.

1845. Nova algarum genera et species, quas in itinere ad oras occidentales Novae Hollandiae, collegit L. Preiss. Botanische Zeitung, $3: 49-57$

1871. Die Algen des tropischen Australiens. Abhandlungen aus dem Gebiete der Naturwissenschaften (Hamburg), 5(2):35-74, 6 plates.

Stacklouse, J.

1797. Nereis Brittanica... Fascicle 2, ix-xxiv + pages 31-70, plates 9-13.

Stafleu, F.A., and R.S. Cowan

1976. Taxonomic Literature ... Volume I: A-G, xI + 1136 pages Utrecht: Boln, Scheltema \& Holkema.

1979. Taxonomic Literature ... . Volume 1I: H-Le, xviii +991 pages. Utrecht: Bohn, Scheltema \& Holkema.

Su, Y.C., J.H. Huang, Y.C. Lin, C.Y. Chen, and F.C. Chen

1982. Pharmacological Properties of Some Taiwan Seaweeds. In R.T. Tsuda and Y.M. Chiang, editors, Proceedings of Republic of ChinaUnited States Seminar on Cultivation and Utilization of Economic Algae, pages 51-53. Mangilao: University of Guam Marine Laboratory

Suringar, W.F.R.

1870. Algae Japonicae musei botanici Lugduno-Batavia. Pages i-viii + 139, 25 plates. Haarlem: Typis Heredum Loosjes.

Tanaka, $\mathrm{T}$

1935. Four New Species of Galaxaura from Japan. Scientific Papers of the Institute of Algological Research, Faculty of Science, Hokkaido Imperial University, 1(1):52-57.

1936. The Genus Galaxaura from Japan. Scientific Papers of the Institute of Algological Research, Faculty of Science, Hokkaido Imperial University, 1(2): 141-174, 41 figures, 12 plates.

1941. The Genus Hypnea from Japan. Scientific Papers of the Institute of 
Algological Research, Faculty of Science, Hokkaido Imperial University, 2(2):227-250.

1944. The Japanese Species of Protoflorideae. Scientific Papers of the Institute of Algological Research, Faculty of Science, Hokkaido Imperial University, 3:79-97, 16 figures.

1950. On the Species of Bangia from Japan. The Botanical Magazine (Tokyo), 63:163-169, 3 figures.

1951. The Species of Erythrotrichia from Japan (1). Acta Phytotaxonomica et Geobotanica, 14:96-100, 1 figure.

1952. The Systematic Study of the Japanese Protoflorideae. Memoirs of the Faculty of Fisheries, Kagoshima University, 2:1-92, plates 1-23.

1967. Some Marine Algae from Bataan and Lamiguru lslands, N. Pliilippines, 1. Memoires of the Faculty of Fisheries, Kagoshima University, 16:13-27, 10 figures, 2 plates.

Tanakd, T., and H. ltono

1969. Studies on the Genus Neurymenia (Rhodomelaceae) from Southern Japan and Vicinities. Memoirs of the Faculty of Fisheries, Kagoshima University, 18:7-27.

Tanaka, T., and K. Nozawa

1962. Some Notes on the Genera Padina and Zonaria in the Southwestern Islands of Japan. Memoirs of the Faculty of Fisheries, Kagoshima University, 11:179-187.

Taniguti, $\mathrm{M}$.

1976. Phytosociological Study of Marine Algae in Taiwan. Bulletin of the Mie University, 27:51-57.

Taylor, W.R.

1945. Pacific Marine Algae of the Allan Hancock Expeditions to the Galapagos Islands. Allan Hancock Pacific Expeditions, 12:iv +528 pages.

1960. Marine Algae of Tropical and Subtropical Coasts of the Americas. ix +870 pages. Amn Arbor: University of Michigan Press.

1962. Two Undescribed Species of Halimeda. Bulletin of the Torrey Botanical Club, 89(3):172-177.

1966. Records of Asian and Western Pacific Marine Algae, Particularly Algae from Indonesia and the Philippines. Pacific Science, 20:342-359

Tilden, J.E.

1929. The Marine and Freshwater Algae of China. Lignan Science Journal, 7:349-399.

Tokida, J.

1939. On Some Edible Seaweeds Utilized among a Native Race "Ami" of Formosa, Collected by Mr. N. Nakanome. Botany and Zoology (Tokyo), 7:1547-1552.

1941. On Some Little Known Marine Algae of Japan, 2. Botany and Zoology (Tokyo), 9:49-56.

1954. The Marine Algae of Southern Saghalien. Memoirs of the Faculty of Fisheries, Hokkaido University, 2(1):1-264, 15 plates.

Trevisan, V.B.A

1845. Nomenclator algarum, ou collection des noms imposés aux plantes de la famille des algues. Volume 1, 80 pages. Padua.

Tseng, C.K.

1936. Studies of the Marine Chlorophyceae from Hainan. Amoy Marine Biology Bulletin, 1(5): 129-200.

1941a. Studies on the Chinese Species of Liagora. Bulletin of the Fan Institute of Biology, Botanical series, 10:265-281.

$194 \mathrm{lb}$. Studies on the Chaetangiaceae of China. Bulletin of the Fan Institute of Biology, Botanical series, 11:83-118.

1944. Marine Algae of Hong Kong, V1: Genus Polysiphonia. Papers of the Michigan Academy of Science, Arts and Letters, 29:67-82.

1945. New and Unrecorded Marine Algae of Hong Kong. Papers of the Michigan Academy of Science, Arts and Letters, 30:157-172.

198 Ia. Marine Phycorulture in China. In T. Levring, editor, Proceedings of the Xth International Seaweed Symposium, pages 123-152. New York: Walter de Gruyter \& Co.

1981b. Commercial Cultivation. In C.S. Lobban and M.J. Wynne, edi- tors, The Biology of Seaweeds, pages 680-725. Berkeley: University of California Press.

Tseng, C.K., editor

1983. Common Seaweeds of China. $\mathrm{x}+316$ pages. Beijing: Science Press.

Tseng, C.K., and C.F. Chang

1961. On the Botanical Names of Economic Marine Algae in Old Clinese Literature. Acta Botanica Sinica, 9:316-336.

Tseng, C.K., and T.J. Chang

1962. Economic Seaweed Flora of China. vii +193 pages, 10 plates. Beijing: Science Press.

Tseng, C.K., Y. Yoshida, and Y.M. Chiang

1985. East Asiatic Species of Sargassum subgenus Bactrophycus J. Agardh (Sargassaceae, Fucales), with Keys to the Sections and Species. In 1.A. Abbott and J.N. Norris, editors, Taxonomy of Economic Seaweeds ...., pages 1-14. La Jolla, California: California Sea Grant College Program, University of California, La Jolla.

Tseng, W.Y.

1984. Eucheuma of Taiwan-Emphasizing Its Mariculture Potential. IIydrobiologia, 116/1 17:237-242.

Tseng, W.Y., and C.S. Chen

1977. Eucheuma of Taiwan. Bulletin of the Taiwan Fisheries Research Institute, 28:103-112.

Ueda, $\mathrm{S}$.

1932. A Systematic Study of Genus Porphyra lound on the Japanese Coasts. Journal of the Imperial Fisheries Institute (Tokyo), 28:145

Umeraki, 1

1972. The Life Histories of Some Nemaliales Whose Tetrasporophytes Were Unknown. In 1.A. Abbott and M. Kurogi, editors, Contributions to the Systematics of Benthic Algae of the North Pacific, pages 231-242. Kobe, Japan: Japanese Society of Phycology.

Vickers, A

1905. Liste des algues marinas du Barbade. Annales des Sciences Naturelles, series $9,1: 45-66$

Voss, E.G., et dl.

1983. International Code of Botanical Nomenclature. [Adopted by the Thirteenth International Botanical Congress, Sydney, August 1981.] xv + 472 pages. Utrecht: Bohn, Scheltema \& Holkema.

Wang, J.C., and Y.M. Chiang

1977. Notes on Marine Algae of Taiwan, 11: The Genus Porphyra. Taiwania, 22:105-112.

Weber-van Bosse, $A$.

1898. Monographie des Caulerpes. Annales du Jardin Botanique de Buitenzorg, 15(2):243-401, 14 plates.

1913a. Liste des algues du Siboga, 1: Myxophyceae, Chlorophyceae, Phaeophyceae. In M. Weber, editor, Siboga-Expeditie: Uitkomsten op Zoologisch, Botanisch, Oceanographisch en Geologisch Gebied .... 59a:1-186, plates 1-52. Leiden: E.J. Brill.

1913b. Marine Algae, Rhodophyceae of the "Sealark" Expedition. Transactions of the Linnean Society, London, series 2 (Botany), 8:105142, plates 12-14.

1921. Liste des algues du Siboga, 11: Rhodophyceze, Part 1: Protoflorideae, Nemalionales, Cryptonemiales. In M. Weber, editor, Siboga-Expeditie: Uitkomsten op Zoologisch, Botanisch, Oceanographisch en Geologisch Gebied . ., 59b:187-310, plates 6-8. Leiden: E.J. Brill.

1928. Liste des algues du Siboga, Part 1V: Rhodophyceae, Part 3 Gigartinales et Rhodymeniales. In M. Weber, editor, SibogaExpeditie: Vitkomsten op Zoologisch, Botanisch, Oceanographisch en Geologisch Gebied ..., 59d:393-533, plates 11-16. Leiden: E.J. Brill.

Wiest, J.A., and M.H. Hommersand

1981. Rhodophyta: Life Histories. In C.S. Lobband and M.J. Wynne, editors, The Biology of Seaweeds, pages 133-193. Berkeley: University of California Press. 
Wiseman, D.R

1975. On the Status of the Red Algal Family, the Rhizophyllidaceae Gigartinales). Taxon, 24:489-490.

Wittrock, V.B.

1866. Försök till en monographie öfver algslägtet Monostroma. 66 pages, 4 plates. Stockholm.

Womersley, H.B.S.

1967. A Critical Survey of the Marine Algae of Southern Australia, 11: Plaeophyta. Australian Journal of Botany, 15:189-270.

1979. Southern Australian Species of Ceramium Roth (Rhodophyta). Australian Journal of Marine and Freshwater Research, 29:205257.

Womersley, H.B.S., and A. Bailey

1970. Marine Algae of the Solomon 1slands. Philosophical Transactions of the Royal Society of London, series B (Biological Sciences), 259(830):257-352.

Wu, C.S

1982. Chemical Analysis and Utilization of Monostroma latissimum. In R.T. Tsudit and Y.M. Chiang, editors, Proceedings of the Republic of China-United States Seminar on Cultivation and Utilization of Economic Algae, pages 55-60. Mangilao: University of Guam Marine Laboratory.

Wynne, M.J.

1985. Nomenclature Assessment of Goniotrichum Kützing, Erythrotricha Areschoug, Diconia Harvey, and Stylonema Reinsch (Rhodophyta). Taxon, 34:502-505.

Wymne, M.J., and G.T. Kraft

1981. Appendix: Classification Summary. In C.S.Lobban and M.J. Wynne, editors, The Biology of Seaweeds, pages 743-750. Berkeley: University of California Press.

Xia, B.M.

1985. Gracilaria from China: Key, List and Distribution of the Species. In I.A. Abbott and J.N. Norris, editors, Taxonomy of Economic Seaweeds, pages 71-76. La Jolla: California Sea Grant College Program, University of California, La Jolla.

Xia, B.M., and H. Yamamoto

1985. Gracilaria Species from Both China and Japan: Key, List, and Distribution of Common and Economically Important Species. In 1.A. Abbott and J.N. Norris, editors, Taxonomy of Economic Seaweeds, pages 69-70. La Jolla: California Sea Grant College Program, University of California, La Jolla.

Xia, B.M., and J.F. Zhang

1982. A New Raw Material for the Manufacture of Agar-agar from Hainan Island, China. Oceanologica et Limnologia Sinica, 13(6):538-543, plate 1 .

Yamada, Y.

1925a. Studien über die Meeresalgen von der lnsel Formosa, 1: Chlorophycese. The Botanical Magazine (Tokyo), 39:77-95, 5 figures.

1925b. Studien über die Meeresalgen von der lnsel Formosa, 11: Phaeophyceae. The Botanical Magazine (Tokyo), 39:239-254, 6 figures.

1926. The Phytogeographical Relation between the Chlorophyceae of the Mariannes, Carolines and Marshall Islands and Those of the Malay Archipelago, Australia and Japan. Proceedings of the Third Pan-Pacific Science Congress. Pages 964-966. Tokyo.

1928. Report of the Biological Survey of Mutsu Bay, 9: Marine Algae of Mutsu Bay and Adjacent Waters, II. Science Report, Tohoku Imperial University, 4th series (Biology), 3:497-534.

1930. Notes on Some Japanese Algae, 1. Scientific Papers of the Institute of Algological Research, Faculty of Science, Hokkaido Imperial University, $1: 27-36$

1931 a. Notes on Some Japanese Algae, I1. Journal of the Faculty of Science, Hokkaido Imperial University, series V, 1(2):65-76, 3 figures, 5 plates.

1931b. Notes on Laurencia, with Special Reference to the Japanese
Species. University of California Publications in Botany, 16:185310,20 figures, 30 plates.

1932a. Notes on Some Japanese Algae, 1Il. Scientific Papers of the Institute of Algological Research, Faculty of Science, Hokkaido Imperial University, 1:109-123.

1932b. Notes on Some Japanese AIgae, IV. Scientific Papers of the Institute of Algological Research, Faculty of Science, Hokkaido Imperial University, 1:267-276.

1933. Notes on Some Japanese Algae, V. Scientific Papers of the Institute of Algological Research, Faculty of Science, Hokkaido Imperial University, 2:277-285.

1934. The Marine Chlorophyceae from Ryukyu, Especially from the Vicinity of Nawa. Journal of the Faculty of Science, Hokkaido Imperial University, series $\mathrm{V}, 3(2): 33-88,55$ figures.

1935. Notes on Some Japanese Algae, V1. Scientific Papers of the Institute of Algological Research, Faculty of Science, Hokkaido Imperial University, 1(1):27-35.

1936a. The Species of Eucheuma from Ryukyu and Formosa. Scientific Papers of the Institute of Algological Research, Faculty of Science, Hokkaido Imperial University, 1(2): 1 19-134, 9 plates.

1936b. Notes on Some Japanese Algae, V1l. Scientific Papers of the Institute of Algological Research, Faculty of Science, Hokkaido Imperial University, 1(2): 135-1 40, 3 figures, 4 plates.

1936c. [Laurencia.] In K. Okamura, Nippon Kaiso-shi [Marine Algae of Japan], pages 851-861. Tokyo: Published by the author.

1938a. The Species of Liagora from Japan. Scientific Papers of the Institute of Algological Research, Faculty of Science, Hokkaido Imperial University, 2:1-34, 22 figures, 15 plates.

1938b. Notes on Some Japanese Algae, V111. Scientific Papers of the Institute of Algological Research, Faculty of Science, Hokkaido Imperial University, 2:119-130.

1941. Notes on Some Japanese Algae, 1X. Scientific Papers of the Institute of Algological Research, Faculty of Science, Hokkaido Imperial University, 2:195-216, 9 plates.

1942. Notes on Sargassum from the Southern Parts of Japan, 1, 11, 111. Japanese Journal of Botany, 18:369-381, 504-519, 553-562.

1944a. Diagnoses of New Sargassums from Japan. Scientific Papers of the Institute of Algological Research, Faculty of Science, Hokkaido Imperial University, 3:1-10.

1944b. Notes on Some Japanese Algae, X. Scientific Papers of the Institute of Algological Research, Faculty of Science, Hokkaido Imperial University, 3:1 1-25, 18 figures.

1944c. A List of the Marine Algae from the Atoll of Ant. Scientific Papers of the Institute of Algological Research, Faculty of Science, Hokkaido Imperial University, 3:31-45.

1950. A List of Marine Algae from Ryukyusho, Formosa, 1: Chlorophyceae and Phaeophyceae. Scientific Papers of the Institute of Algological Research, Faculty of Science, Hokkaido Imperial University, 3:173-194.

Yamada, Y., and S. Segawa

1953. On Some New or Noteworthy Algae from Hachijo 1sland. Records of Oceanographic Works in Japan, new series, 1:109-114.

Yamadiı, Y., and T. Tanaka

1934. Three New Red Algae from Formosa. Transactions of the Natural History Society of Formosa, 24:342-349, 5 figures.

Yamamoto, $\mathrm{H}$.

1985. Gracilaria from Japan: Vegetative and Reproductive Keys and List of the Species. In 1.A. A'obott and J.N. Norris, editors, Taxonomy of Economic Seaweeds, pages 77-80. La Jolla: California Sea Grant College Program, University of California, La Jolla.

Yang, H.N., and Y.M. Chiang

1982. Taxonomical Study on the Gracilaria of Taiwan. Journal of the Fisheries Society of Taiwan, 9:55-71. 
Yang, S.S., C.Y. Wang, and H.H. Wang

1981. Seasonal Variation of Agar-agar Produced in Taiwan Area. In T. Levring, editor, Proceedings of the Xth International Seaweed Yendo, $\mathrm{k}$. Symposium, pages 737-742. New York: Walter de Gruyter \& Co.

1902. Corallinae verae Japonicea. Journal of the College of Science, Imperial University, 16:1-36.

1905. Preliminary List of the Fucaceae of Japan. The Botanical Magazine (Tokyo), 19(222):149-161.

1907. The Fucaceae of Japan. Journal of the College of Science, Imperial University, 21:1-174, 8 plates.

1909. Notes on Algae New to Japan, 1. The Botanical Magazine (Tokyo), 23:117-133.

1914. Notes on Algae New to Japan, 11. The Botanical Magazine (Tokyo), 28:263-281, 1 figure.

1915. Notes on Algae New to Japan, 1II. The Botanical Magazine (Tokyo), 29:99-117.

1916a. Notes on Algae New to Japan, IV. The Botanical Magazine, (Tokyo), 30:47-65.

1916b. Notes on Algae New to Japan, V. The Botanical Magazine (Tokyo), 30:243-263.

1918. Notes on Algae New to Japan, VIII. The Botanical Magazine
(Tokyo), 32:65-81.

1920. Novae algae Japoniae, Decas 1-1II. The Botanical Magazine (Tokyo), 34:1-12.

Yoshikawa, H., and K. Yoshikawa

1977. A List of Marine Algae Collected at Maopit'ou, Taiwan. Joshigaku yo Daigaku Kiyo [Records of the Women's Home Economics College], 8:127-130.

Zanardini, J.

1851. Algae novae vel minus cognitae in mari rubro a Portiero collectae. In Flora oder Allgemaine Botanische Zeitung, Jena, 34(3):3335 .

1858. Plantarum in mari rubro hucusque collectarum enumeratio. Memorie della Istituto Veneto di Scienze, Letere ed Arti, 7:209-309.

1872. Phycearum indicarum pugillus. Memorie della Istituto Veneto di Scienze, Letere ed Arti, 17:129-170.

1878. Phyceae Papuanae Novae vel minus cognitae a Cl. O. Beccari in itinere ad Novam Guineam annis 1872-75 Collectae. Nuovo Giornale Botanico Italiano, 10(1):34-40.

Zhang, J.F. [Chang, C.F.] and B.M. Xia

1985. On Gracilaria asiatica sp. nov. and G. verrucosa (Hudson) Papenfuss. Oceanologia et Limnologica Sinica, 16(3):175-180. 


\section{Index}

Acanthophora

aokii, 22

muscoides, 22

orientalis, 2, 22

Acetabularia

gigas, 11

major, 11

Acetabulariaceae, 11

"Acrocarpus pusillus," 17

Acrochaetiaceae, 15

Acrocystis nana, 22

Actinotrichia fragilis, 15 "rigida," 15

Ahnfeltia paradoxa, 21

Amansia glomerata, 22

Amphiroa

beauvoisii, 18

bowerbankii, 18

“cultrata var. globulifera," 18

ephedraceae, 18

"exilis," 18

fragilissima, 18

multifida, 18

pusilla, 18

"pusilla," 18

"zonata," 18

spp., 18

Anadyomenaceae, 11

Anadyomene wrightii, 11

Asparagopsis

"sanfordiana," 16

taxiformis, 16

"Asterocytis"

"ornata," 14

"ornata f. simplex," 14

"ramosa f. simplex," 14

Bangia

atropurpurea, 14

"fuscopurpurea," 14

yamadae, 14

'yamadai,' 14

sp., 14

Bangiaceae, 14

Bangiales, 14

Boergesenia forbesii, 10

Bonnemaisoniaceae, 16

Boodlea

"bosseae," 10

coacta, 10

composita, 10

montagnei, 10

"pararloxa," 10

siamensis, 10

van-bosseae, 10

'van-Bossei,' 10

Bornetella, 4

sphaerica, 11
Bostrychia tenella, 11

Bryopsidaceae, 10

Bryopsis

harveyana, 10

indica, 10

mucosa, 10

plumosa, 10

Caloglossa bombayensis, 22

Carpoblepharis

schmitziana var, erecta, 21

warburgii, 21

Carpopelt is

angusta, 18

cornea, 18

flabellata, 19

formosana, 2, 19

rigida, 19

Caulacanthus

"fastigiatus," 21

okamurae, 21

'okamurai,' 21

spinellus, 21

ustulatus var. fastigiatus, 1, 21

Caulerpa

cupressoides var. lycopodium f. amicorum, 9

freycinetti var. freycinetti f. lata, 9

"freycinetti var. typica," 9

"peltata," 9

racemosa, 9

"racemosa var. chemnitzia," 9

"racemosa var. clavifera," 9

racemosa var. clavifera $f$. macrophysa, 9

racemosa var. clavifera $f$. microphysa, 9

racemosa var. laetevirens, 9

racemosa var. occidentalis, 9

racemosa var. peltata, 9

racemosa var. turbinata, 9

serrulata, 9

serrulata f. lata, 9

sertularioides, 9

sertularioides $f$. longipes, 9

taxifolia, 9

webbiana, 9

webbiana $\mathrm{f}$. disticha, 9

webbiana f. tomentella, 9

Caulerpaceae, 9

Caulerpales, 8

Centroceras

clavulatum, 21

minutum, 21

Ceramiaceae, 21

Ceramiales, 21

Ceramium

aduncum, 21

ciliatum var, robustum, 21

"clarionense," 21 "clavulatum," 21

"equisetoides," 22

flaccidium, 21

gracillimum, 22

"gracillimum var. byssoideum," 21

nakamurai, 22

paniculatum, 22

tenerrimum, 22

tenuissimum, 22

Ceratodictyon

spongiosum, 20

"spongioides," 20

Chaetangiaceae, 15

Chaetomorpha

aerea, 8

aerea $f$. versata, 8

basiretrorsa, 8

brachygona, 8

crassa, 8

linum, 8

spiralis, 8

Chaetophoraceae, 7

Chaetophorales, 7

Chamaedoris orientalis, 10

Champia parvula, 21

Champiaceae, 21

Cheilosporum

anceps, 18

"cultratum," 18

jungermannioides, 18

Chlorella, 4

Chlorodesmis

caespitosa, 9

comosa, 9

"formosana," 9

Chlorophyta, 1, 7

Chnoospora

implexa, 13

minima, 13

"pacifica," 13

Chondria

armata, 2, 22

dasyphylla, 22

Chondrococcus

hornemannii, 17

'hornemanni,' 17

Chondrus

"affinis," 21

crispus, 21

ocellatus, 21

ocellatus $\mathrm{f}$. canaliculatus, 21

“ocellatus f. typicus," 21

Chordariales, 11

Chroodactylon ornatum, 14

Chrysymenia procumbens, 21

Cladophora

aokii, 8

fuliginosa, 8 
montagnei var. radicans, 8

patentiramea, 8

pellucida, 8

"prolifera," 8

rugulosa, 8

scitula, 8

sibogae, 8

sp., 8

Cladophoraceae, 8

Cladophorales, 8

Cladophoropsis

"fasciculatus," 10

herpestica, 10

sundanensis, 10

zollingeri, 10

Claudea batanensis, 22

Codiaceae, 8

Codium

adhaerens, 8

arabicum, 8

contractum, 8

cylindricum, 8

dichotomum, 8

formosanum, 8

fragile, 8

intricatum, 9

"mucronatum," 8

reediae, 9

repens, 9

tenue, 9

"tomentosum," 9

Colpomenia sinuosa, 13

"Conchocelis," 4

"Corallina"

"adhaerens," 18

"decussato-dichotoma," 18

Corallinaceae, 2, 18

"Corallopsis opuntia," 20

Corynothlaeacea, 11

Cryptonemiaceae, 18

Cryptonemiales, 17

Cystoseira

prolifera, 13

sp., 13

Cystoseiraceae, 13

Dasya sp., 22

Dasyaceae, 22

Dasycladales, 11

Delesseriaceae, 22

Dasyphila plumarioides, 22

Delisea

fimbriata, 16

"japonica," 16

Derbesia lamourouxii, 10

Derbesiaceae, 10

"Dermatolithon tumidulum," 18

Dermonema

frappieri, 15

gracile, 15

dichotoma, 2

"dichotomum," 15

pulvinata, 15

Dermonemataceae, 15

"Desmia hornemannii," 17
Dictyopteris

delicatula, 11

repens, 11

undulata, 11

Dictyosphaeria

bokotensis, 10

cavernosa, 10

"favulosa," 10

Dictyota

bartayresii, 11

ceylanica var. anastomosans, 12

dichotoma, 12

dilata, 12

divaricata, 12

hamifera, 12

linearis, 12

patens, 12

spinulosa, 12

Dictyotaceae, 11

Dictyotales, 11

Digenia simplex, 22

Dilophus

okamurae, 12

'okamurai,' 12

"marginatus," 12

radicans, 12

Dotyophycus yamadae, 15

Dudresnaya japonica, 17

Dumontiaceae, 17

Ectocarpaceae, 11

Ectocarpales, 11

Ectocarpus

breviarticulatus, 11

"formosanus," 11

laurenciae, 11

"mitchelliae," 11

spinosus, 11

vanbossea, 11

van-bossea, 11

sp., 11

Endarachne binghamiae, 13

"Endocladia complanata," 18

Endocladiaceae, 18

Endophyton ramosum, 7

Enteromorpha

clathrata, 7

clathrata var. crinita, 7

"crinita," 7

compressa, 7

intestinalis, 7

linza, 7

prolifera, 7

sp., 7

Erythrocolon podagrica, 21

Erythropeltidaceae, 14

Erythrotrichia

biseriata, 14

carnea $f$. tenuis, 14

Eucheuma

arnoldii, 19

"audiolis," 19

cottonii, 19

“cottonii," 19

crassum, 19 crustaeforme, 19

"cupressoideum," 19

gelatinae, 19

muricatum, 19

"muricatum," 19

muricatum $\mathrm{f}$. depauperata, 19

okamurae, 19

'okamurai,' 19

papulosa, 19

serra, 19

"spinosum," 19

spp., 19

Feldmannia formosana, 11

Fucales, 13

Galaxaura, 2

arborea, 15

canaliculata, 16

clavigera, 16

distenta, 16

elegans, 16

elongata, 16

falcata, 16

fasciculata, 16

fastigiata, 16

filamentosa, 16

lapidescens, 16

lapidescens f. villosa, 16

latifolia, 16

marginata, 16

oblongata, 16

obtusata, 16

pacifica, 16

robusta, 16

rudis, 16

rugosa, 16

"tenera," 16

veprecula, 16

Ganonema, 15

Gelidiaceae, 17

Gelidiella

acerosa, 17

Gelidiellaceae, 17

Gelidiopsis

hachijoensis, 20

repens, 20

"rigida," 17

variabilis, 20

Gelidium, 2, 3, 4

amansii, 17

amansii f. elegans, 17

amansii $\mathrm{f}$. latioris, 17

"cartilagineum," 17

"clavatum," 17

“corneum var. pinnatum," 17

crinale, 17

"densum," 17

divaricatum, 17

japonicum, 17

kintaroi, 17

latiusculum, 17

pacificum, 17

planiusculum, 17

pusillum, 17 
pusillum $\mathrm{f}$. foliaceum, 17

subcostatum, 17

yamadae, 17

sp., 17

Giffordia mitchelliae, 11

Gigartina

intermedia, 21

ochotensis, 21

tenella, 21

Gigartinaceae, 21

Gigartinales, 19

Gloiopeltis

cervicornis, 18

complanata, 18

furcata, 18

tenax, 18

Goniotrichaceae, 14

Goniotrichales, 14

"Goniotrichum alsidii," 14

Gracilaria, 4

arcuata, 20

blodgettii, 20

bursapastoris, 20

canaliculata, 20

chorda, 20

"compressa," 20

"confervoides," 20

coronopifolia, 20

crassa, 20

denticulata, 20

edulis, 4, 20

eucheumioides, 20

gigas, 4, 20

incurvata, 20

"lichenoides," 20

"lichenoides f. coronopifolia," 20

punctata, 20

purpurascens, 20

"purpurascens f. spinulosa," 20

salicornia, 20

spinulosa, 20

"verrucosa," 4, 20

sp., 21

Gracilariaceae, 20

Grateloupia

carnosa, 19

filicina, 19

filicina $\mathrm{f}$. filiformis, 1,19

"filiformis," 19

okamurae, 19

“okamurai," 19

ramosissima, 19

Gymnogongrus

flabelliformis, 21

"paradoxus," 21

"Gymnosorus collaris," 12

Gymnothamnion elegans, 22

Halimeda

cuneata, 9

discoidea, 9

incrassata var. ovata, 9

macroloba, 9

opuntia, 9

"opuntia f. intermedia," 9 “opuntia f. renschii," 9

renschii, 9

velasquezii, 9

"Haliseris undulata," 11

Haloplegma duperreyi, 22

Halymenia

ceylanica, 19

durvillaei, 19

“durvillaei var. formosa," 2, 19

"durvillaei var. ceylanica," 19

"formosa," 19

Helminthocladia australis, 15

Herposiphonia

subdisticha, 22

sp., 22

Holmesia neurymenioides, 22

"Homoeostrichus"

"flabellatus," 12

"multifidus," 12

Hormophysa triquetra, 13

Hydroclathrus

"cancellatus," 13

clathratus, 13

"orientalis," 13

Hypnea, 2

boergesenii, 19

cenomyce, 19

cervicornis, 19

charoides, 19

chordacea, 19

chordacea f. simpliciuscula, 19

cornuta, 19

esperi, 19

hamulosa, 19

japonica, 20

nidulans, 20

pannosa, 20

saidana, 20

seticulosa, 2, 20

spinella, 20

sp., 20

Hypneaceae, 19

"1lea fascia," 12

lshige

okamurae, 12

'okamurai,' 12

sinicola, 12

"foliacea," 12

lshigeaceae, 12

Jania

adhaerens, 18

"decussato-dichotoma," 18

radiata, 18

tenella, 18

undulata, 18

Laurencia, 2

brongniartii, ii, 22

“dasyphylla," 22

flexilis var. tropica, 23

forsteri, 23

glandulifera, 23 grevilleana, 23

obtusa var. densa, 23

palisada, 23

papillosa, 23

perforata, 23

pinnatifida, 23

"pinnatifida var. simplex," 23

tropica, 23

venusta, 23

sp., 2, 23

"Letterstedtia japonica," 8

Leveillea jungermannioides, 23

Liagora, 2, 4

“annulata," 15

boergesenii, 15

"caenomyce," 15

ceranoides, 15

ceranoides var. leprosa, 15

ceranoides var. pulverulenta, 15

decussata, 15

farinosa, 15

"formosana," 15

"maxima," 15

orientalis, 15

"orientalis," 15

rugosa, 15

segawai, 15

setchellii, 15

valida, 15

Liagoraceae, 15

Liagorophila endophytica, 15

Liagoropsis schrammi, 15

Lithophyllum

perulatum, 18

spp., 18

Lithothamnium

membranaceum, 18

spp., 18

Lobophora variegata, 12

Martensia

denticulata, 22

flabelliformis, 22

Mastophora

pygmaea, 18

rosea, 18

"macrocarpa," 18

Melobesia

farinosa, 18

"membranaceae," 18

"pustulata," 18

spp., 18

Meristotheca

coacta, 19

papulosa, 19

Microcladia elegans, 22

Microdictyon

japonicum, 10

nigrescens, 10

okamurae, 10

Monostroma, 4

latissimum, 7

nitidum, 8 
Murrayella periclados, 23

squarrosa, 23

Nemaliales, 15

"Nemalion pulvinatum," 2, 15

Neomeris, 4 annulata, 11

"Neurocarpus" "repens," 11

"undulata," 11

"undulata f. plana," 11

Neurymenia fraxinifolia, 23

Nitophyllum sp., 22

Nostoc, 3

Pachydictyon coriaceum, 12

Padina

arborescens, 12

australis, 12

boryana, 12

commersonii, 12

"commersonii," 12

crassa, 12

durvillaei, 12

japonica, 12

minor, 12

"pavonia," 12

tenuis, 12

Petalonia fascia, 12

Peyssonnelia

caulifera, 18

distenta, 18

"involvens," 18

rubra, 18

Peyssonneliaceae, 18

Phacelocarpus japonicus, 21

Phaeophyta, 1, 11

"Phycoseris"

"lanceolata var. angusta," 7

"lapathifolia," 8

"Phyllitis fascia," 12

Phyllophoraceae, 21

"Placophora marchantioides," 23

Plocamiaceae, 20

Plocamium

"abnorme," 20

"abnorme f. uncinatum," 20

"costatum," 20

oviforme, 20

serratulum, 20

telfairiae, 20

telfairiae $f$. uncinatum, 20

"Plumaria ramosa," 22

Polyopes

polyideoides, 19

sp., 19

Polysiphonia

harlandii, 23

kampsaxii, 23

pulvinata, 23

"Polyzonia jungermannioides," 23

Porphyra, 2, 4

angusta, 14

crispata, 14 dentata, 15

suborbiculata, 15

sp., 15

Pterocladia, 2, 3

"capillaceum," 17

nana, 17

tenuis, 17

Ptilothamnion cladophorae, 22

Punctariaceae, 12

Reinboldiella schmitziana, 22

"Rhipidiphyllon"

"reticulatum," 10

"nigrescens," 10

Rhizophyllidaceae, 17

Rhodoglossum affine, 21

Rhodomelaceae, 22

Rhodopeltis

borealis, 17

gracilis, 17

setchelliae, 17

'setchelii,' 17

Rhodophyta, 1, 14

Rhodymenia spinulosa, 21

Rhodymeniaceae, 21

Rhodymeniales, 21

Rosenvingea orientalis, 13

Sarcodiaceae, 21

Sarcodia ceylanica, 21

Sargassaceae, 13

Sargassum, 2, 3, 4

amabile, 13

aquifolium, 13

baccularia, 13

berberifolium, 13

binderi, 13

"biserrula," 13

carpophyllum, 13

coriifolium, 13

coriifolium f. duplicatum, 13

coriifolium $\mathrm{f}$. prolongatum, 13

crassifolium, 13

crispifolium, 13

cristaefolium, 13

duplicatum, 4, 13

echinocarpum, 13

fulvellum, 13

glaucescens, 13

hemiphyllum, 13

henslowianum, 13

heterocystum, 13

horneri, 13

ilicifolium, 13

ilicifolium var. conduplicatum, 13

kasyotense, 14

kuetzingii, 14

kushimotense, 14

"microphyllum," 14

muriocystum, 14

plagiophyllum, 14

polycystum, 14

"prolongatum," 13

rostratum, 14 sandei, 14

sandei f. heterophyllum, 14

serratifolium, 14

siliquosum, 14

swartzii, 14

telephifolium, 14

tenuifolium, 14

vulgare, 14

sp., 14

Scinaia

boergesenii, 16

cottonii, 16

moniliformis, 16

pseudojaponica, 16

Scytosiphon

lomentaria, 13

'lomentarium,' 13

"lomentarius," 13

Scytosiphonaceae, 13

Scytosiphonales, 12

Siphonocladaceae, 10

Siphonocladales, 10

Solieriaceae, 19

Spatoglossum pacificum, 12

"Spermothamnion"

"cladophorae," 22

"codicola," 22

Sphacelaria

furcigera var. tenuis, 11

tribuloides, 1 I

Sphacelariaceae, 11

Sphacelariales, 11

Sphaerococcaceae, 21

Spyridia filamentosa, 22

Struvea

anastomosans, 10

delicatula, 10

Symphyocladia marchantioides, 23

Tenarea tumidulum, 18

Tiffaniella codicola, 22

Turbinaria

conoides, 14

filamentosa, 14

"filiformis," 14

ornata, 14

trialata, 14

sp., 14

Udoteaceae, 9

Ulothrix flaccida, 7

Ulotrichaceae, 7

Ulotrichales, 7

Ulva

angusta, 8

conglobata, 8

fasciata, 8

japonica, 8

lactuca, 8

lactuca f. lapathifolia, 1, 8

pertusa, 8

reticulata, 8

rigida, 8

sp., 8

Ulvaceae, 7

Ulvales, 7 


\author{
Valonia \\ aegagropila, 11 \\ "confervoides," 11 \\ fastigiata, 11 \\ "forbesii," 10 \\ utricularis, 11 \\ verticillata, 11 \\ Valoniaceae, 10 \\ Valoniopsis pachynema, 11
}

Vidalia obtusiloba, 23

Weberella micans, 21

Wrangelia velutina, 22

Wurdemannia

setacea, 23

sp., 23

Wurdemanniaceae, 23

Yamadaella cenomyce, 15
Zonaria

coriacea, 12

diesingiana, 12

flabellata, 12

harveyana, 12

nigrescens, 12

stipitata, 12

"variegata," 12

sp., 12 




\section{REQUIREMENTS FOR SMITHSONIAN SERIES PUBLICATION}

Manuscripts intended for series publication receive substantive review (conducted by their originating Smithsonian museums or offices) and are submitted to the Smithsonian Institution Press with Form SI-36, which must show the approval of the appropriate authority designated by the sponsoring organizational unit. Requests for special treatment-use of color, foldouts, case-bound covers, etc.-require, on the same form, the added approval of the sponsoring authority.

Review of manuscripts and art by the Press for requirements of series format and style, completeness and clarity of copy, and arrangement of all material, as outlined below, will govern, within the judgment of the Press, acceptance or rejection of manuscripts and art.

Copy must be prepared on typewriter or word processor, double-spaced, on one side of standard white bond paper (not erasable), with 11/4" margins, submitted as ribbon copy (not carbon or xerox), in loose sheets (not stapled or bound), and accompanied by original art. Minimum acceptable length is 30 pages.

Front matter (preceding the text) should include: title page with only title and author and no other information, abstract page with author, title, series, etc., following the established format; table of contents with indents reflecting the hierarchy of heads in the paper; also, foreword and/or preface, if appropriate.

First page of text should carry the title and author at the top of the page; second page should have only the author's name and professional mailing address, to be used as an unnumbered footnote on the first page of printed text.

Center heads of whatever level should be typed with initial caps of major words, with extra space above and below the head, but no other preparation (such as all caps or underline, except for the underline necessary for generic and specific epithets). Run-in paragraph heads should use period/dashes or colons as necessary.

Tabulations within text (lists of data, often in parallel columns) can be typed on the text page where they occur, but they should not contain rules or numbered table captions.

Formal tables (numbered, with captions, boxheads, stubs, rules) should be submitted as carefully typed, double-spaced copy separate from the text; they will be typeset unless otherwise requested. If camera-copy use is anticipated, do not draw rules on manuscript copy.

Taxonomic keys in natural history papers should use the aligned-couplet form for zoology and may use the multi-level indent form for botany. If cross referencing is required between key and text, do not include page references within the key, but number the keyed-out taxa, using the same numbers with their corresponding heads in the text.

Synonymy in zoology must use the short form (taxon, author, year:page), with full reference at the end of the paper under "Literature Cited." For botany, the long form (taxon, author, abbreviated journal or book title, volume, page, year, with no reference in "Literature Cited") is optional.

Text-reference system (author, year:page used within the text, with full citation in "Literature Cited" at the end of the text) must be used in place of bibliographic footnotes in all Contributions Series and is strongly recommended in the Studies Series: "(Jones, 1910:122)" or ". . Jones (1910:122)." If bibliographic footnotes are required, use the short form (author, brief title, page) with the full citation in the bibliography.

Footnotes, when few in number, whether annotative or bibliographic, should be typed on separate sheets and inserted immediately after the text pages on which the references occur. Extensive notes must be gathered together and placed at the end of the text in a notes section.

Bibliography, depending upon use, is termed "Literature Cited," "References," or "Bibliography." Spell out titles of books, articles, journals, and monographic series. For book and article titles use sentence-style capitalization according to the rules of the language employed (exception: capitalize all major words in English). For journal and series titles, capitalize the initial word and all subsequent words except articles, conjunctions, and prepositions. Transliterate languages that use a non-Roman alphabet according to the Library of Congress system. Underline (for italics) titles of journals and series and titles of books that are not part of a series. Use the parentheses/colon system for volume (number): pagination: "10(2):5-9." For alignment and arrangement of elements, follow the format of recent publications in the series for which the manuscript is intended. Guidelines for preparing bibliography may be secured from Series Section, SI Press.

Legends for illustrations must be submitted at the end of the manuscript, with as many legends typed, double-spaced, to a page as convenient.

Illustrations must be submitted as original art (not copies) accompanying, but separate from, the manuscript. Guidelines for preparing art may be secured from Series Section, SI Press. All types of illustrations (photographs, line drawings, maps, etc.) may be intermixed throughout the printed text. They should be termed Figures and should be numbered consecutively as they will appear in the monograph. If several illustrations are treated as components of a single composite figure, they should be designated by lowercase italic letters on the illustration; also, in the legend and in text references the italic letters (underlined in copy) should be used: "Figure $9 \mathrm{~b}$." Illustrations that are intended to follow the printed text may be termed Plates, and any components should be similarly lettered and referenced: "Plate $9 \underline{b}$." Keys to any symbols within an illustration should appear on the art rather than in the legend.

Some points of style: Do not use periods after such abbreviations as "mm, ft, USNM, NNE." Spell out numbers "one" through "nine" in expository text, but use digits in all other cases if possible. Use of the metric system of measurement is preferable; where use of the English system is unavoidable, supply metric equivalents in parentheses. Use the decimal system for precise measurements and relationships, common fractions for approximations. Use day/month/year sequence for dates: "9 April 1976." For months in tabular listings or data sections, use three-letter abbreviations with no periods: "Jan, Mar, Jun," etc. Omit space between initials of a personal name: "J.B. Jones."

Arrange and paginate sequentially every sheet of manuscript in the following order: (1) title page, (2) abstract, (3) contents, (4) foreword and/or preface, (5) text, (6) appendixes, (7) notes section, (8) glossary, (9) bibliography, (10) legends, (11) tables. Index copy may be submitted at page proof stage, but plans for an index should be indicated when manuscript is submitted. 


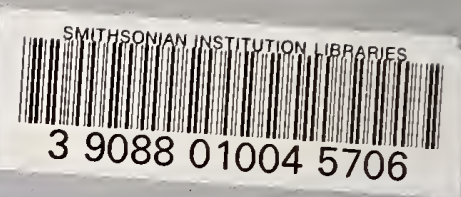

$x^{2}-x^{2}=y^{2}$ 